\title{
A multi-country test of brief reappraisal interventions on emotions during the COVID-19 pandemic
}

\begin{abstract}
The COVID-19 pandemic has increased negative emotions and decreased positive emotions globally. Left unchecked, these emotional changes might have a wide array of adverse impacts. To reduce negative emotions and increase positive emotions, we tested the effectiveness of reappraisal, an emotion-regulation strategy that modifies how one thinks about a situation. Participants from 87 countries and regions $(n=21,644)$ were randomly assigned to one of two brief reappraisal interventions (reconstrual or repurposing) or one of two control conditions (active or passive). Results revealed that both reappraisal interventions (vesus both control conditions) consistently reduced negative emotions and increased positive emotions across different measures. Reconstrual and repurposing interventions had similar effects. Importantly, planned exploratory analyses indicated that reappraisal interventions did not reduce intentions to practice preventive health behaviours. The findings demonstrate the viability of creating scalable, low-cost interventions for use around the world.
\end{abstract}

\section{Protocol registration}

The stage 1 protocol for this Registered Report was accepted in principle on 12 May 2020. The protocol, as accepted by the journal, can be found at https://doi.org/10.6084/m9.figshare.c.4878591.v1

T he COVID-19 pandemic is increasing negative emotions and decreasing positive emotions around the globe ${ }^{1-10}$. Concurrently, individuals are reporting that COVID-19 is having a negative impact on their psychological functioning and mental health ${ }^{4,11,12}$. For example, individuals report sleeping less, consuming more alcohol or other drugs or substances, having trouble concentrating because their mind is occupied by COVID19 , and having more fights with their partner or loved ones, some escalating to domestic violence ${ }^{1,9,13}$.

These disturbing trends are caused partly by heightened levels of negative emotion and diminished levels of positive emotion, which have been found to contribute to a number of negative psychological, behavioural and health consequences. These include increased risk of anxiety and depressive disorders as well as other forms of psychopathology ${ }^{14}$; impaired social connections ${ }^{15}$; increased substance use $^{16-18}$; compromised immune system functioning ${ }^{19-21}$; disturbed sleep ${ }^{22}$; increased maladaptive eating ${ }^{23,24}$; increased aggressive behaviour ${ }^{25,26}$; impaired learning ${ }^{27}$; worse job performance ${ }^{28,29}$; and impaired economic decision-making ${ }^{30,31}$.

As the COVID-19 pandemic unfolds around the world, we believe it is crucial to mitigate expected adverse outcomes by reducing negative emotions and increasing positive emotions. Such a change in emotions is central to increasing psychological resilience, a multifaceted concept that involves adaptive emotional responses in the face of adversity ${ }^{32-34}$. Reappraisal-an emotion regulation strategy that involves changing how one thinks about a situation with the goal of influencing one's emotional response ${ }^{35}$-is a promising candidate as an intervention to increase psychological resilience due to its adaptability, simplicity and efficiency ${ }^{34,36-38}$. In contrast to less effective emotion-regulation strategies such as suppression, reappraisal generally leads to more successful regulation $(d=0.45,95 \%$ confidence interval $(\mathrm{CI})=[0.35,0.56]$ in changing emotion experience in a meta-analysis ${ }^{39}$; see caveats about interpreting effect sizes in past research in Methods, 'Sampling plan'). In particular, over the short term, reappraisal leads to decreased reports of negative emotion and increased reports of positive emotion ${ }^{40-42}$, as well as corresponding changes both in peripheral physiological responses ${ }^{43-45}$ and central physiological responses ${ }^{46-53}$. Over the longer term, reappraisal is associated with stronger social connections ${ }^{54}$; higher academic achievement ${ }^{55,56}$; enhanced psychological well-being ${ }^{57}$; fewer psychopathological symptoms $\mathrm{s}^{58,59}$; better cardiovascular health ${ }^{60,61}$, and greater resilience during the COVID-19 pandemic ${ }^{62}$.

Despite these shorter-term and longer-term benefits, most people do not reappraise consistently ${ }^{63,64}$, which has motivated efforts to teach people to use reappraisal (reviewed in refs. ${ }^{65,66}$ ). For example, in the context of anxiety, reappraisal training led to reduced intrusive memories ${ }^{67}$ and increased emotion-regulation self-efficacy ${ }^{68,69}$. Reappraisal training also led to long-lasting changes in the neural representation of unpleasant events ${ }^{70}$.

Although demand characteristics are always a concern when examining the effects of reappraisal (given that one is teaching people to change their thinking in order to change how they're feeling, and then asking them how they feel $)^{71}$, the wide array of self-report and non-self-report outcomes ${ }^{39-53}$ that show reappraisal effects across studies increases confidence that these effects are real. It is also encouraging to note that reappraisal generally outperforms other types of emotion regulation such as suppression, even though demand characteristics appear comparable across regulation conditions $^{39}$. In addition, evidence indicates that reappraisal interventions can influence emotional outcomes even in intensely challenging contexts in which people are often unmotivated to regulate their emotions $^{72}$. For example, a brief reappraisal training conducted in the context of the Israeli-Palestinian conflict and replicated in the context of the Colombian conflict ${ }^{73}$, has been found to contribute to reduced intergroup anger and increased support for conciliatory political policies ${ }^{74}$.

As part of the attempt of the Psychological Science Accelerator (PSA) to address pressing questions related to the psychological 
impact of COVID-19, the current study aimed to use reappraisal interventions to enhance psychological resilience in response to the pandemic. To maximize the impact of these interventions, this project had a global reach of large, diverse samples via the PSA's network $^{75}$, and employed highly scalable methods that were translated for use around the world. In order to make stronger and clearer inferences, our design included two reappraisal interventions that were compared with two control conditions, an active control and a passive control.

For our reappraisal interventions, we examined two theoretically defined forms of reappraisal ${ }^{76}$ - reconstrual and repurposing. Reconstrual involves changing how a situation was construed or mentally represented in a way that changes the emotional responses related to the situation. Examples of reconstrual in response to COVID-19 include: "Washing hands, avoiding touching my face, keeping a safe distance... There are simple and effective things I can do to protect myself and my loved ones from getting sick and to stop the spread of the virus" and "I know from world history that keeping calm and carrying on gets us through tough times". Repurposing involves focusing on a potentially positive outcome that could come from the current situation in a way that changes the emotional response to it. Examples of repurposing in response to COVID19 include: "This situation is helping us realize the importance of meaningful social connections, and helping us understand who the most important people in our lives are" and "Medical systems are now learning to deal with amazing challenges, which will make them much more resilient in the future". For our active control condition, we asked participants to reflect on their thoughts and feelings as they unfolded. Meta-analyses have revealed that reflecting on one's thoughts and feelings produces small but reliable salutary effects $(d=0.07,9 \% \mathrm{CI}=[0.05,0.17]$ in improving psychological health, including emotional responses ${ }^{77,78}$ ). Examples of reflecting in response to COVID-19 are: "I really wish we could find a vaccine soon" and "This situation is changing so fast, and I don't know how the future will develop". By asking participants in this condition to actively use a strategy that is likely to have a positive effect, we sought to match expectancy and demand across reappraisal and active control conditions. For our passive control condition, we asked participants to respond as they naturally do, which is a commonly used passive control condition in prior research on emotion regulation (for a meta-analysis, see ref. ${ }^{39}$ ).

In comparing conditions, we chose to distinguish between negative and positive emotional responses, as previous evidence suggests that the two are clearly separable $e^{79,80}$. Specifically, we hypothesized that our reappraisal interventions would lead to reduced negative emotional responses (hypothesis 1) and increased positive emotional responses (hypothesis 2) compared with both control conditions combined. While both reconstrual and repurposing strategies involve changing thinking, we hypothesized that the reconstrual intervention would lead to greater decreases in negative emotional responses than the repurposing intervention (hypothesis 3) and that the repurposing intervention would lead to greater increases in positive emotional responses than the reconstrual intervention (hypothesis 4). We theorized that reconstruing one's situation should primarily decrease negative emotions, because it typically focuses on ameliorating the problem at hand. The reconstrual intervention is most similar to a previously studied subtype of reappraisal called reappraising emotional stimulus, which has been investigated mainly on negative emotions and has a $d=0.38$ and $95 \% \mathrm{CI}=[0.21$, $0.55]$ for changing emotion experience ${ }^{39}$. Repurposing one's situation, by contrast, should primarily increase positive emotions because it usually calls to mind positive experiences. Repurposing is similar to a few previously examined types of reappraisals, such as benefit finding and positive reappraisal, both of which are primarily associated with positive outcomes ${ }^{81,82}$ (Methods, 'Sampling plan' provides further detail).
In testing these hypotheses, we planned to use orthogonal contrasts that make two primary comparisons, while keeping all other comparisons exploratory (Table 1 provides further detail). The first comparison contrasted both the reappraisal conditions combined with both the active control condition and the passive control condition combined for negative (hypothesis 1 ) and positive (hypothesis 2) emotions. The second comparison contrasted the reconstrual and repurposing interventions for negative (hypothesis 3 ) and positive (hypothesis 4) emotions. One attractive feature of comparisons between reappraisal conditions is that there is no reason to assume that demand or expectancies would differ across these reappraisal conditions.

One potential concern about the current design was that the emotion-regulation interventions might reduce preventive health behaviours (for example, maintaining social distance and washing hands) that could potentially be motivated by negative emotions. Some research on the connection between emotions and health behaviour suggests that increased negative emotions such as fear do not seem to be a strong motivator for changing one's health behaviour $^{83}$. Furthermore, positive emotions augmented by the reappraisal interventions may contribute to a greater tendency to undertake health behaviours ${ }^{84,85}$. For example, positive emotions can lead to higher medication adherence ${ }^{86}$. To ensure that our interventions would not adversely impact any relevant health behaviours, we took two steps. First, during the instructions, we clarified that-in some cases-negative emotions such as fear and sadness may be helpful, and that it is up to each person to determine when an emotion is unhelpful or not and to downregulate only those emotions that are unhelpful. Second, to assess whether our training would lead to reduced vigilance, we specifically measured and examined intentions to follow stay-at-home orders and wash hands in exploratory analyses.

In addition, we conducted other exploratory analyses. These analyses included testing the impact of our reappraisal interventions on negative and positive anticipated emotions and intentions to enact potentially harmful versus beneficial behaviours associated with these emotions (details described in Methods, 'Measures'), and assessed whether the effects of our reappraisal interventions, if any, were moderated by motivation to use the given strategy $y^{71}$, belief in the effectiveness of the given strategy ${ }^{87}$, or demographics (gender ${ }^{39}$, socioeconomic status ${ }^{88,89}$ or country or region ${ }^{90}$ (hereafter country/region) (particularly in light of the differing levels of impact of COVID-19 in any given country/region at any given point in time)).

\section{Results}

Final sample size and demographics. We collected 27,989 responses between May 2020 and October 2020. After implementing preregistered exclusions (see detail in https://doi.org/10.6084/ m9.figshare.c.4878591.v1) and an additional exclusion of nine duplicate IDs, our final sample included 21,644 participants from 87 countries/regions $(63.41 \%$ female, $35.34 \%$ male, $0.45 \%$ other genders, $0.56 \%$ preferred not to say and $0.24 \%$ missing responses to the gender question; participants were aged $31.91 \pm 14.52 \mathrm{yr}$ (mean \pm s.d.); see Supplementary Table 1 for sample size per country/region and Supplementary Table 2 for sample size per month). Of the 87 countries/regions represented, 37 had more than 200 participants, surpassing our $95 \%$ power criterion based on simulations in our power analysis (see detail in Methods, 'Power analysis').

We preregistered two exclusion criteria. First, as planned, we excluded participants who answered both multiple choice manipulation check questions incorrectly, and found that conditions had similar proportions of such participants $(0.55 \%)$, Holm's adjusted $P$ values $>0.999$. Second, as planned, we excluded participants who completed fewer than $50 \%$ of the questions in the study, and found that the passive control condition had fewer such participants $(16.17 \%)$ than the other three conditions $(23.86 \%$ in the 


\begin{tabular}{|c|c|c|c|c|}
\hline & $\begin{array}{l}\text { Active } \\
\text { control }\end{array}$ & $\begin{array}{l}\text { Passive } \\
\text { control }\end{array}$ & Reconstrual & Repurposing \\
\hline $\begin{array}{l}\text { Contrast } 1 \\
\text { (hypotheses 1-2) }\end{array}$ & 0.5 & 0.5 & -0.5 & -0.5 \\
\hline $\begin{array}{l}\text { Contrast } 2 \\
\text { (hypotheses 3-4) }\end{array}$ & 0 & 0 & 0.5 & -0.5 \\
\hline
\end{tabular}

active control condition, $24.41 \%$ in the reconstrual condition and $23.90 \%$ in the repurposing condition), Holm's adjusted $P<0.001$. One possible explanation for this difference is that the instructions given to participants in the passive control condition were shorter than those given in the other conditions, requiring less cognitive effort to read and less time to complete the study. Applying both exclusion criteria, the overall exclusion rate was significantly lower in the passive control condition (16.71\%) than in the other three conditions $(24.47 \%$ in the active control condition, $24.99 \%$ in the reconstrual condition and $24.37 \%$ in the repurposing condition), Holm's adjusted $P<0.001$. To rule out concerns related to differences in exclusion rates, we repeated all preregistered analyses on the full sample. Reassuringly, all patterns, statistical significance and conclusions remained unchanged when analyses were repeated on the full sample (Supplementary Table 3).

Preregistered analyses. We included all 87 countries/regions in all analyses regardless of their sample sizes, except for Fig. 1, Supplementary Fig. 1 and Supplementary Fig. 2, where the 37 countries/regions with $n \geq 200$ were analysed separately by country/ region. Effect sizes, frequentist statistics and Bayes factors for each of our hypotheses are presented in Table 2. Raw means and standard deviations for each relevant measure are provided in Table 3. Details of analytical models are described in Methods.

Hypotheses regarding the shared effects of two brief reappraisal interventions. Consistent with the main hypotheses of the study, both reappraisal interventions combined (versus both control conditions combined) significantly decreased negative emotional responses (hypothesis 1) and significantly increased positive emotional responses (hypothesis 2) across all primary outcome measures (emotions in response to the photos related to COVID-19 from various news sources, state emotions after viewing all the photos and emotions about the COVID-19 situation; Table 2, rows 2-7; details of these measures are described in Methods). As shown in Fig. 1, this finding was consistent across the 37 countries/regions in which there were more than 200 participants (although all 87 countries/ regions were included in the analysis testing hypotheses regardless of their sample size, only the 37 countries/regions with $n \geq 200$ were analysed separately by country/region for Fig. 1). For example, in comparing participants' immediate negative emotional responses to the photos related to the COVID-19 situation, data from 33 out of the 37 (89\%) countries/regions showed significant effects of the reappraisal interventions in the hypothesized direction. None of the 37 countries/regions' data revealed a statistically significant result in the opposite direction.

Hypotheses regarding the unique effects of the two reappraisal interventions. Results revealed little to no support for our hypotheses regarding the differences between reconstrual and repurposing, as neither was reliably better than the other at reducing negative emotions or increasing positive emotions across outcomes (Table 2, rows 8-13; Supplementary Fig. 2). We hypothesized that reconstrual would produce greater decreases in negative emotional responses than repurposing (hypothesis 3 ), and data revealed supportive evidence for only one outcome (negative emotions about the COVID19 situation; Table 2, row 10) out of the three measures of negative emotions. The other two negative emotion outcome measures did not support that hypothesis. One outcome (negative emotions in response to the photos; Table 2, row 8) revealed that repurposing had significantly stronger effects in decreasing negative emotional responses than reconstrual, whereas the Bayes factor indicated inconclusive evidence. Another outcome (negative state emotions; Table 2, row 9) revealed no significant difference between types of reappraisal, and the Bayes factor indicated strong evidence in favour of the null hypothesis.

We also hypothesized that repurposing would produce greater increases in positive emotional responses than reconstrual (hypothesis 4), and data revealed supportive evidence for only one outcome (positive emotions in response to the photos; Table 2, row 11) out of the three measures of positive emotions. The other two outcome measures of positive emotions revealed no significant differences between the two reappraisal conditions. The Bayes factors indicated strong evidence in favour of the null hypothesis for one outcome (positive state emotions; Table 2, row 12) and inconclusive evidence for another outcome (positive emotions about the COVID-19 situation; Table 2, row 13). Overall, there were no consistent differences across outcomes between reconstrual and repurposing in reducing negative emotions or increasing positive emotions in the current experimental context. We examined potential reasons for these findings in the exploratory analyses and in the discussion section.

Exploratory analyses. To better understand the impact of the reappraisal interventions, we conducted four sets of exploratory analyses. First, we examined pairwise comparisons between conditions (each of the reappraisal conditions versus each of the control conditions, and the active control condition versus the passive control condition) for our primary outcomes (emotions in response to the photos, state emotions after viewing all the photos and emotions about the COVID-19 situation). Second, we assessed the effect of reappraisal interventions on four exploratory outcomes (behavioural intentions to practice preventive health behaviours, participants' engagement with emotion regulation strategies, global change in emotions, and anticipated emotions). Third, we assessed four sets of potential moderators of reappraisal interventions' effects (motivation to use the given strategy ${ }^{71}$, belief in the effectiveness of the given strategy ${ }^{87}$, demographics ${ }^{39,88-90}$ and lockdown status). Finally, we contextualised reappraisal interventions' effect sizes on negative emotions by comparing them with effect sizes of lockdown status and self-isolation due to symptoms. Details of analytical models are reported in Supplementary Information (Supplementary Tables 4 and 5).

Pairwise comparisons of conditions on primary outcomes. In the first set of exploratory analyses, we examined the extent to which each of the reappraisal conditions differed from each of the control conditions for our primary outcomes (emotions in response to the photos, state emotions after viewing all the photos and emotions about the COVID-19 situation). Pairwise comparisons for all primary outcomes produced results consistent with the pattern of evidence for hypothesis 1 and hypothesis 2 . Each of the repurposing and reconstrual conditions (versus each of the control conditions) significantly decreased negative emotional responses and significantly increased positive emotional responses $(P s<0.001$; Table 3$)$.

We also examined whether the active and passive control conditions differed from each other at the level of pairwise comparisons. Among the three primary outcome measures of negative emotional responses, one was significantly higher in the active control condition than in the passive control condition (negative emotions 
Emotions in response to the photos related to COVID-19 "How negative (positive) did the photo make you feel?"

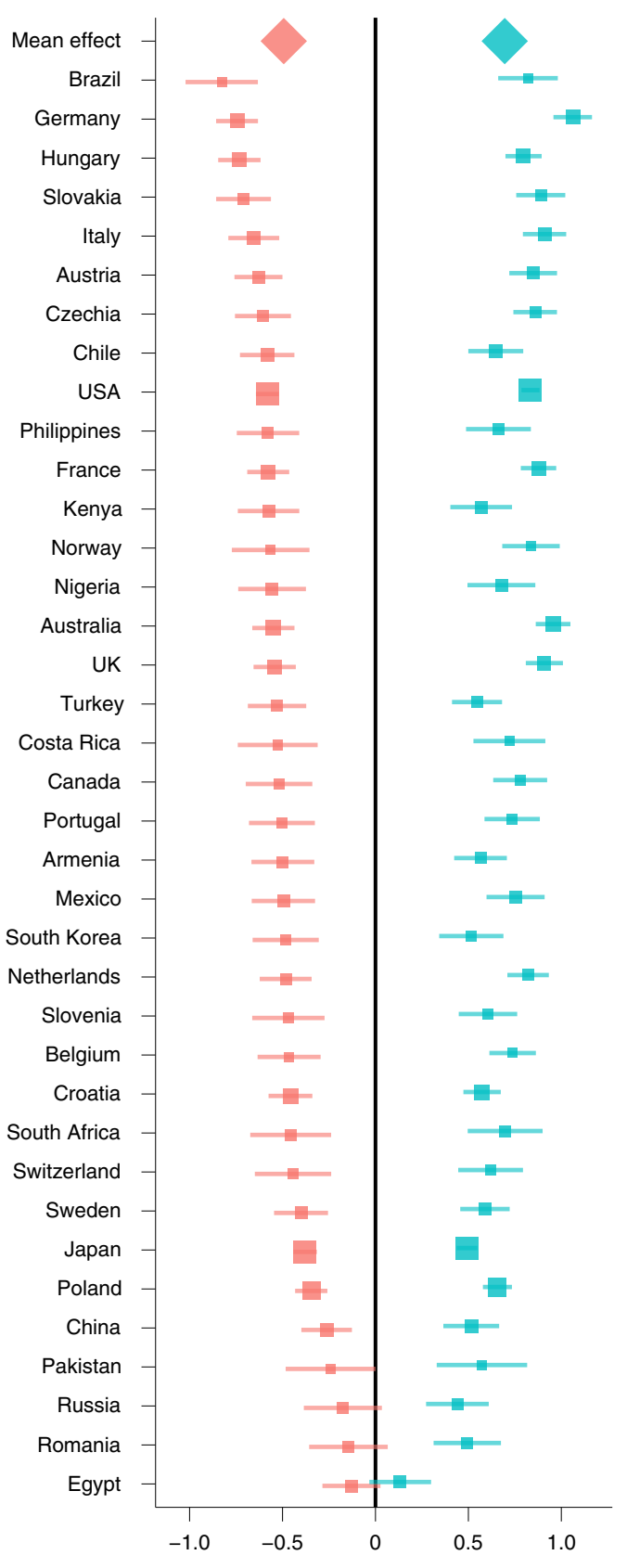

State emotions after viewing all the photos related to COVID-19 "How are you feeling right now?"

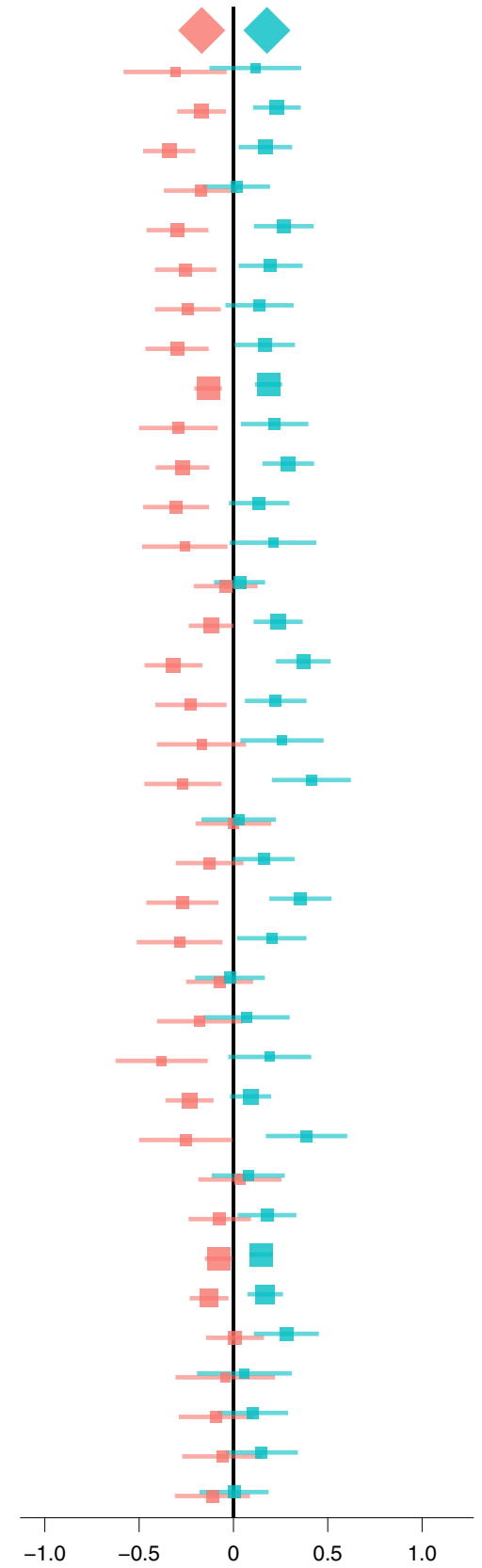

Emotions about the COVID-19 situation "Overall, how negative/hopeless (positive/hopeful) are you feeling about the COVID-19 situation right now?"

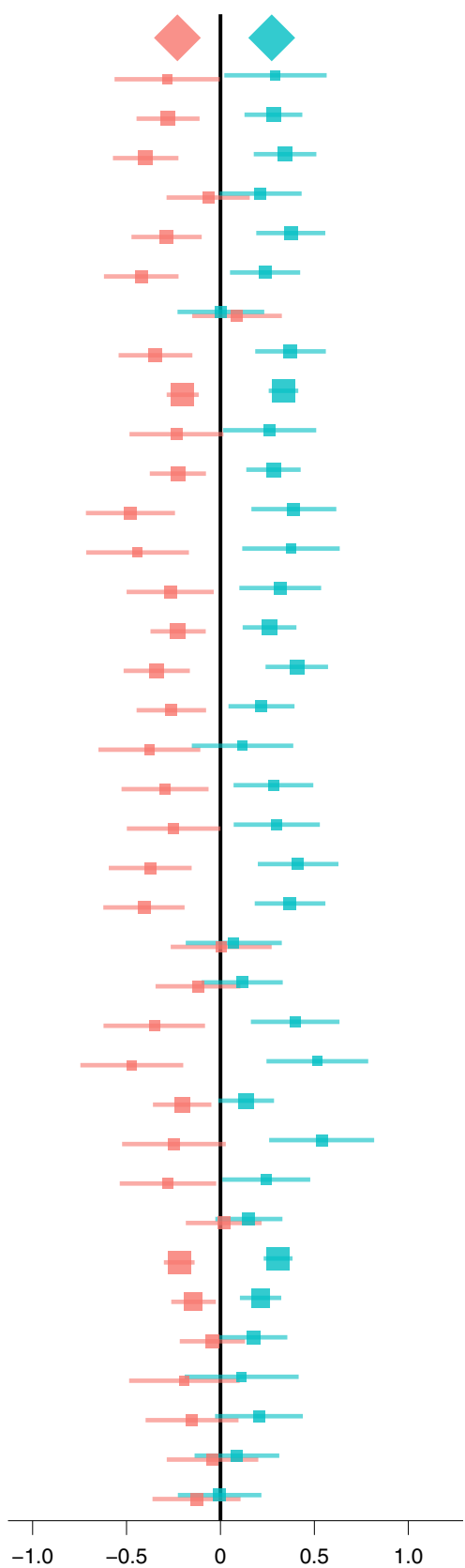

Both reappraisal interventions combined (versus both control conditions combined) rating differences on 5 -point scales with $95 \%$ confidence interval

Fig. 1 | Effect sizes of both reappraisal interventions combined (versus both control conditions combined) on primary outcomes by country/region. In almost all of the 37 countries/regions in which there were more than 200 participants, both reappraisal interventions combined (versus both control conditions combined) decreased negative emotional responses and increased positive emotional responses for primary outcome measures (emotions in response to the photos, state emotions after viewing all the photos, and emotions about the COVID-19 situation). Effect sizes are raw mean differences on five-point scales without adjusting for covariates. Confidence intervals are based on the $t$ distribution. Countries/regions are ordered by decreasing effect sizes of negative emotions in response to the photos, and larger dots reflect larger samples (Supplementary Fig. 1 presents the countries/regions in alphabetical order.). 


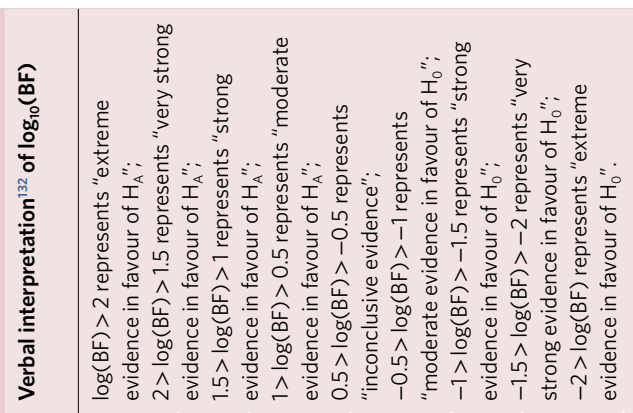

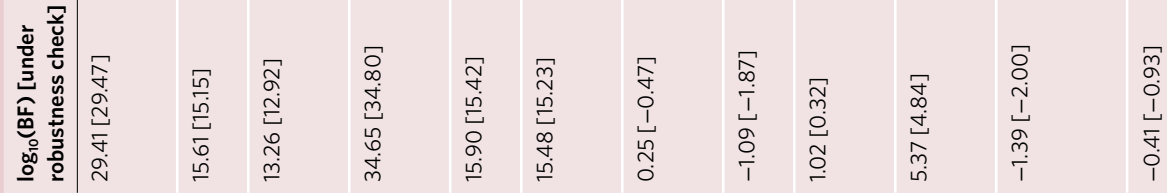

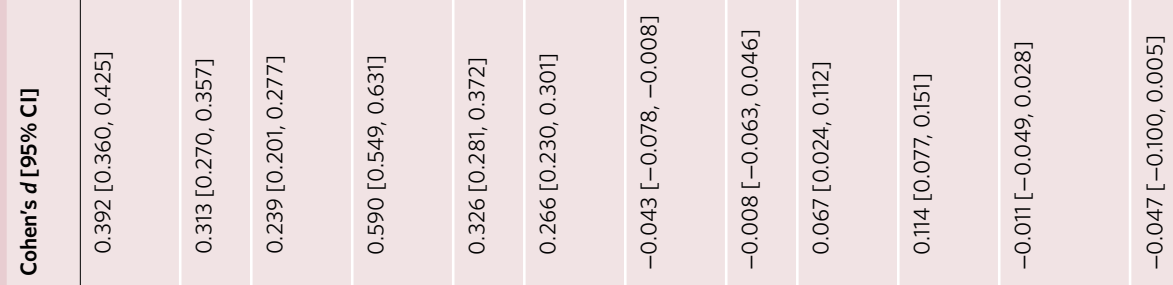

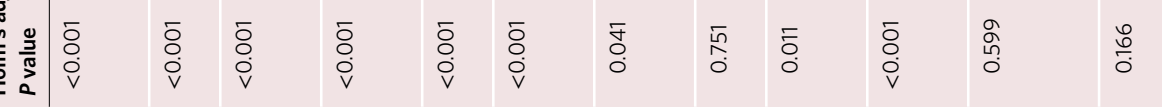

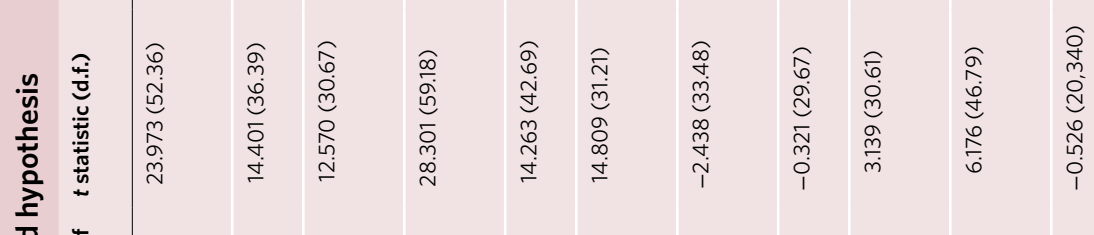


Table 3 | Raw mean and s.d. values for outcomes

\begin{tabular}{|c|c|c|c|c|}
\hline \multirow[t]{2}{*}{ Outcome } & \multicolumn{2}{|c|}{ Reappraisal interventions } & \multicolumn{2}{|c|}{ Control conditions } \\
\hline & $\begin{array}{l}\text { Reconstrual } \\
(n=5,078)\end{array}$ & $\begin{array}{l}\text { Repurposing } \\
(n=5,421)\end{array}$ & $\begin{array}{l}\text { Active control } \\
(n=5,349)\end{array}$ & $\begin{array}{l}\text { Passive control } \\
(n=5,796)\end{array}$ \\
\hline \multicolumn{5}{|l|}{ Primary outcomes } \\
\hline Negative emotions in response to the photos & $2.77^{\mathrm{a}}(0.80)$ & $2.71^{b}(0.77)$ & $3.29^{\circ}(0.83)$ & $3.19^{d}(0.84)$ \\
\hline Positive emotions in response to the photos & $2.47^{\mathrm{a}}(0.81)$ & $2.62^{b}(0.79)$ & $1.86^{\mathrm{c}}(0.72)$ & $1.84^{\mathrm{d}}(0.73)$ \\
\hline Negative state emotions & $2.32^{\mathrm{a}}(0.90)$ & $2.31^{\mathrm{a}}(0.90)$ & $2.52^{\mathrm{b}}(0.95)$ & $2.48^{b}(0.95)$ \\
\hline Positive state emotions & $3.17^{\mathrm{a}}(0.88)$ & $3.18^{\mathrm{a}}(0.87)$ & $2.99^{\mathrm{b}}(0.88)$ & $2.98^{b}(0.90)$ \\
\hline Negative emotions about the COVID-19 situation & $2.71^{\mathrm{a}}(1.08)$ & $2.77^{b}(1.07)$ & $2.99^{c}(1.10)$ & $2.97^{c}(1.10)$ \\
\hline Positive emotions about the COVID-19 situation & $2.91^{\mathrm{a}}(1.05)$ & $2.88^{\mathrm{a}}(1.04)$ & $2.65^{b}(1.06)$ & $2.59^{\circ}(1.06)$ \\
\hline \multicolumn{5}{|l|}{ Exploratory outcomes } \\
\hline Intention to follow stay-at-home orders stringently & $5.42^{\mathrm{a}}(1.79)$ & $5.44^{\mathrm{a}}(1.77)$ & $5.41^{\mathrm{a}}(1.80)$ & $5.45^{\mathrm{a}}(1.77)$ \\
\hline Intention to wash hands regularly for at least $20 \mathrm{~s}$ & $5.82^{\mathrm{a}}(1.53)$ & $5.82^{\mathrm{ab}}(1.50)$ & $5.82^{\mathrm{ab}}(1.51)$ & $5.76^{b}(1.56)$ \\
\hline Frequency of natural response & $3.49^{\mathrm{a}}(1.35)$ & $3.53^{b}(1.35)$ & $4.00^{c}(1.17)$ & $4.56^{d}(0.79)$ \\
\hline Frequency of using reflecting & $3.92^{\mathrm{a}}(1.11)$ & $3.90^{\mathrm{a}}(1.14)$ & $4.25^{b}(0.97)$ & $3.91^{\mathrm{a}}(1.20)$ \\
\hline Frequency of using reconstrual & $3.80^{\mathrm{a}}(1.09)$ & $3.73^{b}(1.14)$ & $3.06^{c}(1.27)$ & $2.75^{d}(1.34)$ \\
\hline Frequency of using repurposing & $3.89^{\mathrm{a}}(1.13)$ & $4.15^{b}(1.01)$ & $3.21^{c}(1.31)$ & $3.12^{\mathrm{d}}(1.34)$ \\
\hline Motivation to use the given strategy & $6.14^{\mathrm{a}}(1.12)$ & $6.17^{\mathrm{a}}(1.12)$ & $6.26^{b}(1.04)$ & $6.43^{c}(1.00)$ \\
\hline Belief in the effectiveness of the given strategy & $5.00^{\mathrm{a}}(1.68)$ & $5.03^{a}(1.69)$ & $4.80^{b}(1.76)$ & $4.44^{c}(1.90)$ \\
\hline Global change in negative feelings & $2.82^{\mathrm{a}}(0.94)$ & $2.75^{b}(0.93)$ & $3.19^{\circ}(0.92)$ & $3.17^{c}(0.88)$ \\
\hline Global change in positive feelings & $3.28^{\mathrm{a}}(0.91)$ & $3.33^{\mathrm{a}}(0.91)$ & $2.92^{b}(0.92)$ & $2.92^{b}(0.89)$ \\
\hline Anticipated negative emotions & $2.31^{\mathrm{a}}(0.90)$ & $2.30^{\mathrm{a}}(0.89)$ & $2.45^{b}(0.92)$ & $2.44^{b}(0.94)$ \\
\hline Anticipated positive emotions & $3.26^{\mathrm{a}}(0.88)$ & $3.26^{\mathrm{a}}(0.87)$ & $3.13^{b}(0.86)$ & $3.11^{b}(0.89)$ \\
\hline
\end{tabular}

Values are displayed as raw mean (s.d.). Sample sizes ( $n$ ) presented in the second row reflect the numbers of participants after preregistered exclusion. Sample sizes vary by outcome because we dropped incomplete cases on an analysis-by-analysis basis. All primary outcomes were assessed on five-point scales. The following four exploratory outcomes were assessed on seven-point scales: intention to follow stay-at-home orders stringently, intention to wash hands regularly for at least $20 \mathrm{~s}$, motivation to use the given strategy, and belief in the effectiveness of the given strategy. The remaining exploratory outcomes were assessed on five-point scales. Within each row, means that do not share a superscript differ at $P<0.05$; two-tailed, Holm's method for adjustment. For instance, means both marked with ${ }^{\text {a }}$ do not differ significantly, but means marked with $^{\mathrm{a}}$ and $^{\mathrm{b}}$ differ significantly from each other.

in response to the photos: $B=0.091 \pm 0.015, t(20,740)=6.192$, $P<0.001, d=0.070,95 \% \mathrm{CI}=[0.048,0.093])$, while the other two showed no significant differences (negative state emotions: $B=0.022 \pm 0.011, t(20,400)=1.933, P=0.053, d=0.037$, $95 \% \mathrm{CI}=[-0.001,0.075]$; negative emotions about the COVID-19 situation: $B=0.005 \pm 0.022, t(26.01)=0.221, P=0.827, d=0.005$, $95 \% \mathrm{CI}=[-0.040,0.047])$. Among the three primary outcome measures of positive emotional responses, two were significantly higher in the active control condition than in the passive control condition (positive emotions in response to the photos: $B=0.039 \pm 0.013$,

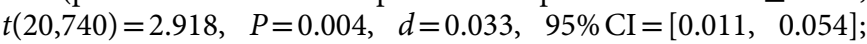
positive emotions about the COVID-19 situation: $B=0.053 \pm 0.019$, $t(233.7)=2.805, \quad P=0.005, \quad d=0.053, \quad 95 \% \mathrm{CI}=[0.015, \quad 0.091])$, while one showed no significant differences (positive state emotions: $B=0.009 \pm 0.010, t(20,350)=0.858, P=0.391, d=0.017$, $95 \% \mathrm{CI}=[-0.021,0.054])$. Thus, effects produced by the active control condition versus the passive control condition differed infrequently. When they did differ, differences were small in magnitude, inconsistent in direction, and slightly smaller in effect size than was suggested by previous meta-analyses ${ }^{77}(d=0.07$, $95 \% \mathrm{CI}=[0.05,0.17])$.

Effects of reappraisal interventions on four exploratory outcomes. Details of exploratory outcomes can be found in Methods and Fig. 2. Descriptive statistics and pairwise comparisons for exploratory outcomes can be found in Table 3. Here we focus on the contrast between the two reappraisal interventions combined and the two control conditions combined.
Behavioural intentions to practice preventive health behaviours. To address the concern that reappraisal interventions might reduce preventive health behaviours (by reducing negative emotions such as fear), we asked about participants' behavioural intentions to follow stay-at-home orders stringently and to wash their hands regularly for at least $20 \mathrm{~s}$ the following week. We found that reappraisal interventions (versus both control conditions combined) did not significantly change intentions to follow stay-at-home orders $(B=0.009 \pm 0.024, \quad t(15.04)=0.38, P=0.709, \quad d=0.005$, $95 \% \mathrm{CI}=[-0.023,0.032])$ or to wash hands $(B=0.034 \pm 0.020$, $t(20,740)=1.69, P=0.091, d=0.022,95 \% \mathrm{CI}=[-0.004,0.048])$. Pairwise comparisons revealed that the only significant difference was that participants in the reconstrual condition reported higher intentions to wash their hands than those in the passive control condition $(B=0.077 \pm 0.028, t(20,740)=2.714$, Holm's adjusted $P=0.040, d=0.051,95 \% \mathrm{CI}=[0.014,0.087])$. These results thus provide preliminary evidence that reappraisal interventions did not significantly reduce intentions to practice preventive health behaviours.

Participants' engagement with emotion-regulation strategies. To better understand participants' engagement with emotion-regulation strategies when viewing the photos related to COVID-19, we examined participants' self-reported frequency of using different strategies when viewing the photos, motivation to use their given strategy, and belief in the effectiveness of their given strategy.

Providing confidence in the effectiveness of the manipulation, we found that participants in each of the four conditions reported 


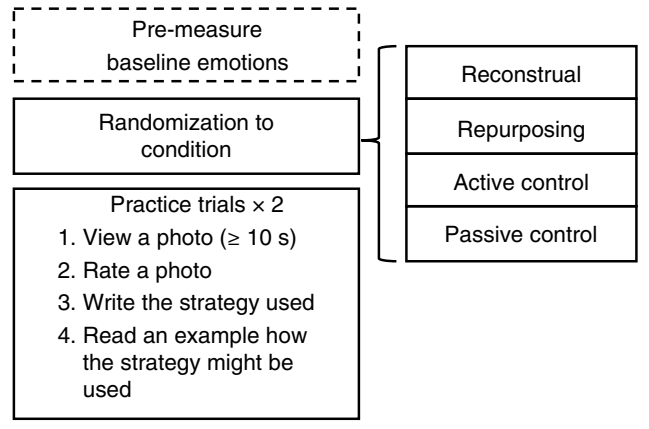

\begin{tabular}{|l|}
\hline $\begin{array}{l}\text { Experimental trials } \times 10 \\
\text { 1. View a photo }(\geq 10 \mathrm{~s}) \\
\text { 2. Rate a photo }\end{array}$ \\
1. State emotions \\
2. Emotions about the \\
COVID-19 situation \\
3. Anticipated emotions \\
4. Behavioural intentions \\
6. Motivation/beliefs \\
1
\end{tabular}

Fig. 2 | Overview of the experiment. Participants in the passive control condition did not have the fourth step in the practice trials.

using the strategy instructed in their condition more frequently than using the other strategies (see Table 3). It is noteworthy that participants in the two reappraisal conditions reported using both reconstrual and repurposing more frequently than those in either control conditions rather than primarily using only the form of reappraisal instructed in their condition. This finding may help explain the lack of differences between the two reappraisal conditions on our primary outcomes.

Next, we examined participants' motivation to follow the given instructions, as well as participants' belief that the given strategy could influence their emotions. We found that participants in the two reappraisal interventions (versus both control conditions combined) reported being significantly less motivated to follow their given instructions while viewing the photos $(B=-0.192 \pm 0.016$, $t(20.87)=-11.62, \quad P<0.001, d=-0.183, \quad 95 \% \mathrm{CI}=[-0.215$, $-0.152])$, but reported significantly greater belief in the effectiveness of their given strategy $(B=0.420 \pm 0.053, t(52.05)=7.97$, $P<0.001, d=0.233,95 \% \mathrm{CI}=[0.175,0.290])$. Thus, the reappraisal conditions were effective in changing emotions despite the fact that participants in reappraisal conditions reported being less motivated to follow the instructions than participants in the control conditions.

Global change of emotions. At the end of the study, we asked participants how they felt compared with at the beginning of the study. We found that reappraisal interventions (versus both control conditions combined) significantly reduced global negative feelings $(B=-0.397 \pm 0.026, t(45.29)=15.30, P<0.001, d=-0.432$, $95 \% \mathrm{CI}=[-0.489,-0.377])$ and significantly increased global positive feelings $(B=0.378 \pm 0.023, t(45.49)=16.75, \quad P<0.001$, $d=0.423,95 \% \mathrm{CI}=[0.373,0.473])$. These findings suggest that the effects are not specific to items in the immediate proximity of the manipulations.

Anticipated emotions. To gain insight into the potential longer-term effects of reappraisal interventions, we asked participants how they anticipated they would feel the following week. We found that reappraisal interventions (versus both control conditions combined) significantly reduced negative anticipated emotions $(B=-0.125 \pm 0.012, \quad t(41.99)=-10.27, \quad P<0.001, \quad d=-0.205$, $95 \% \mathrm{CI}=[-0.245,-0.166])$ and significantly increased positive anticipated emotions $(B=0.125 \pm 0.008, t(13.07)=15.58, P<0.001$, $d=0.227,95 \% \mathrm{CI}=[0.197,0.256])$. These findings suggest that participants anticipated that reappraisal strategies would be useful in improving their emotional well-being in the future.

Exploratory moderators of intervention effects. Prior research suggests that emotion-regulation interventions lead to better results when the participants are: motivated to regulate their emotions ${ }^{71}$, led to believe in the effectiveness of regulation ${ }^{87}$, female (versus male $)^{39}$, from lower (versus higher) socioeconomic status ${ }^{88,89}$, and from Western (versus Eastern) cultures $^{90}$. We examined these as well as lockdown status (as a proxy for differing levels of impact of COVID-19) as potential moderators on our primary outcomes (emotions in response to the photos, state emotions after viewing all the photos, and emotions about the COVID-19 situation).

Controlling for baseline emotions, results of multilevel models revealed that two of the variables moderated intervention effects across all six primary outcomes. Specifically, the higher the scores on motivation to use the given strategy and on belief in the effectiveness of the given strategy were, the more effective the interventions were (Supplementary Figs. 3 and 4 and Supplementary Tables 6 and 7). Two variables (gender and employment status) moderated intervention effects on four of the six primary outcomes: Females (versus males) and individuals who had no employment and no income (versus those who had employment and income or versus those with no employment but with income) showed stronger effects of the intervention (Supplementary Tables 9 and 10). One variable moderated intervention effects on two of the six outcomes: the higher a country/region scored on Hofstede's ${ }^{91}$ index of individualism, the more effective the intervention was in increasing positive emotions in response to the photos and increasing positive emotions about the COVID-19 situation among participants from that country/region (Supplementary Table 8). Subjective socioeconomic status, education level, and lockdown status significantly moderated no more than one of the six outcomes, which would be unlikely to hold after correction for multiple comparisons (Supplementary Tables 11-13). Full, detailed results are reported in the Supplementary Information.

Contextualising reappraisal interventions' effect sizes. To facilitate interpretation of reappraisal effect sizes, it is helpful to compare them to effect sizes of other factors that may have also contributed to differences in participants' emotions. One such candidate for comparison is differences in emotional experience as a function of lockdown status and of self-isolation due to symptoms. Assuming that lockdown or self-isolation due to symptoms impacted participants' emotions, emotional changes caused by these factors could be compared to the ones caused by our interventions in order to get a sense of the impact of our intervention.

With negative state emotions as the outcome variable, we examined lockdown status and self-isolation due to symptoms, respectively, as a fixed variable in two separate multilevel models with random by-country/region slopes and random by-country/ region intercepts to estimate the pure effect size of each variable (as lockdown status and self-isolation due to symptoms were correlated, entering both variables simultaneously in the same model may generate biased estimates). We found that participants whose areas were in full lockdown reported more negative state emotions than participants whose areas were not in lockdown $(B=0.154 \pm 0.040, \quad t(37.56)=3.812, P<0.001, d=0.159$, $95 \% \mathrm{CI}=[0.075,0.243])$, and participants whose areas were in partial lockdown reported more negative state emotions than participants 
whose areas were not in lockdown $(B=0.094 \pm 0.027, t(27.25)=$ $3.531, P=0.001, d=0.097,95 \% \mathrm{CI}=[0.041,0.155])$. We also found that participants who were self-isolating due to flu-like or cold-like symptoms reported more negative state emotions than participants who were not self-isolating due to flu-like or cold-like symptoms $(B=0.175 \pm 0.044, t(25.83)=3.981, P<0.001, d=0.183$, $95 \% \mathrm{CI}=[0.092,0.283])$. As shown in Table 2 for hypothesis $1 b$, participants who were in the two reappraisal conditions reported less negative state emotions than participants who were in the two control conditions $(B=0.185 \pm 0.013, t(36.39)=14.401, P<0.001$, $d=0.313,95 \% \mathrm{CI}=[0.270,357])$. In addition, the amount of variance explained by fixed effects in a model with only lockdown status as a fixed variable is marginal ${ }^{92} R^{2}=0.003$. The amount of variance explained by fixed effects in a model with only self-isolation due to symptoms as a fixed variable is marginal ${ }^{92} R^{2}=0.001$. The amount of variance explained by fixed effects in a model with only the contrast between the two reappraisal conditions and the two control conditions as the fixed variable is marginal ${ }^{92} R^{2}=0.013$. Across different measures of effect size, it is notable that the effects of reappraisal interventions on state negative emotions were of similar or even larger magnitude than the effects of lockdown status or self-isolation due to symptoms. This comparison suggests that reappraisal interventions could help to alleviate the emotional toll caused by lockdown and self-isolation. Thus, we believe that the effects of reappraisal interventions are not only statistically significant but also practically meaningful.

\section{Discussion}

The current study had two main goals. The first was to examine the shared effects of two brief reappraisal interventions (versus both passive and active control conditions) on negative and positive emotions in response to the COVID-19 pandemic, and to determine whether these effects were similar or different across countries/ regions and COVID-19 situations. The second goal was to examine the potentially unique effects of the two reappraisal interventionsreconstrual and repurposing-on negative and positive emotions.

Regarding the first goal, we predicted and found that both reappraisal interventions (versus both control conditions combined) consistently decreased negative emotional responses (hypothesis 1) and consistently increased positive emotional responses (hypothesis 2) across all primary outcome measures: immediate emotions in response to each photo about the COVID-19 situation, state emotions after viewing all the photos related to the COVID-19 situation and overall emotions about the COVID-19 situation. Exploratory analyses suggested that both reappraisal interventions also improved participants' reported emotions compared with at the beginning of the study and the emotions they anticipated feeling in the future.

Further exploratory analyses suggested that despite substantial local variations in how severe the pandemic was at the time data were collected and cultural differences in how people understand and respond to emotions $s^{90,93}$, the intervention effects appeared in almost all of the countries/regions we studied. For example, in comparing participants' immediate negative emotional responses to the photos related to the COVID-19 situation, 33 out of the 37 (89\%) countries/regions with high statistical power (over 200 participants) showed statistically significant effects of reappraisal interventions. Although reappraisal interventions tended to have larger effects among females (versus males), and among unemployed individuals without income, the effects were largely unqualified by education level, subjective socioeconomic status, and whether a participant's country/region was under lockdown.

Regarding the second goal, we predicted that reconstrual would be more effective at reducing negative emotions than repurposing (hypothesis 3), but repurposing would be more effective at increasing positive emotions than reconstrual (hypothesis 4). We found little to no support for these hypotheses, as neither was reliably better than the other at reducing negative emotions or increasing positive emotions across outcomes. The finding that the two forms of reappraisal were similarly effective at regulating emotions in the context of COVID-19 is consistent with the idea that the pandemic offers a wide array of affordances both for construing emotional situations in different ways, thus enabling reconstrual, and for evaluating these situations in light of different goals, thus enabling repurposing ${ }^{76}$. This implies that it may be beneficial to combine both strategies, a hypothesis that future studies can be designed to test. It also remains to be investigated whether reconstrual and repurposing offer similarly comparable benefits in other contexts.

The comparable effectiveness of reconstrual and repurposing in this context raises interesting questions about these two forms of reappraisal. We found that even though participants learned only one form of reappraisal, they reported using both strategies more often than in either control condition. This overlap might have stemmed from insufficient differentiation between the reappraisal instructions used in this study. It may also mean that the distinction between repurposing and reconstrual, although useful theoretically, is not readily accessible to lay people. Alternatively, this overlap may have stemmed from reconstrual and repurposing being mutually associated to a degree that being instructed to use one strategy primes the other strategy. Future research is needed to more directly investigate these possibilities.

After assessing results related to the primary goals, an important question was whether reducing negative emotions and increasing positive emotions in response to the pandemic might inadvertently come at the cost of decreasing intentions to engage in preventive health behaviour (reviewed in ref. ${ }^{94}$ ). Reassuringly, the reappraisal interventions improved emotions without significantly reducing intentions to practice preventive health behaviours. This is consistent with recent findings that there are many paths to motivate preventive health behaviours during the COVID-19 pandemic without inducing negative emotions ${ }^{95-98}$.

Our results highlight the benefits of applying reappraisal interventions at scale to increase psychological resilience and to mitigate the adverse impacts of the COVID-19 pandemic-benefits that could potentially be applied in other contexts that elicit negative emotions. Importantly, the effects of the intervention were not meagre: the extent to which emotions were changed by our reappraisal interventions was comparable in magnitude to the extent to which emotions differed between people who faced extreme hardships (lockdowns or symptom-induced isolations) and people who experienced neither of these hardships. Thus, contextualising the effect sizes of reappraisal interventions in this manner suggests that the interventions are practically meaningful. This practical meaning matters in light of findings that people on average do not appear to fully recover their emotional well-being even after six months into the COVID-19 pandemic ${ }^{99}$, that stress and depression can impair vaccine efficacy ${ }^{100}$, and that negative emotions predispose morbidity and mortality via increases in substance use and other risky behaviours ${ }^{101}$. Essential workers, nurses and doctors, students, patients and many other populations whose work and life are highly affected by the pandemic could potentially benefit from reappraisal interventions, although more research is needed to establish the effectiveness of reappraisal for groups facing distinct challenges. Because these interventions are inexpensive, brief and scalable, they could be implemented through a variety of media and communication mechanisms, such as advertising campaigns ${ }^{102}$, speeches, courses, apps and mobile games ${ }^{103}$.

Our results also have important implications for the science of emotion (reviewed in ref. ${ }^{104}$ ) and for emotion regulation (reviewed in refs. ${ }^{35,39}$ ) in particular. Despite the fact that reappraisal is one of the most researched topics in psychology ${ }^{35}$, this study is the largest cross-cultural investigation of reappraisal that has been conducted 
to date, drawing diverse samples from well beyond the WEIRD (western, educated, industrialized, rich and democratic) societies $^{105}$ that have been heavily represented to date in social science. Thus, the findings reveal the generalizability of reappraisal effects across many countries/regions even in the context of substantial, protracted stressors. The present study also extends understanding of how contextual moderators influence reappraisal processes (for example, individualism, lockdown status and demographics) while deepening understanding of distinct forms of reappraisal (that is, comparing them in relation to multiple outcomes). Finally, our study provides a rich dataset for examining many other questions related to emotions, emotion regulation and cultural differences. We look forward to seeing what other insights can be generated from this dataset.

Despite the encouraging findings, several limitations should be noted. One limitation is the use of convenience sampling and a limited set of photos. Our sample was not nationally representative within each country/region, and it appeared to over-represent females, younger people and people with internet access. The photos used in the study, although carefully chosen, might not represent local situations for different groups of participants. Future research is needed to assess generalizability using nationally representative samples and more personally emotionally evocative stimuli. A second limitation is that we cannot fully rule out the influence of demand characteristics and expectancies. Although we attempted to match demand characteristics and expectancies in the reappraisal conditions using our active control condition, we did not quantify the extent to which they were comparable, and we measured perceived strategy effectiveness after participants had used the strategies, which is different from expectancies formed upon reading the instructions but before using the strategies. Future research should assess the influence of demand characteristics and expectancies. A third limitation relates to the fact that the current study examined only the immediate and proximal effects of the interventions. Future research employing longitudinal designs is needed to examine whether the effects persist over time and at what intervals individuals might optimally engage in reappraisal. A fourth limitation is that the current study examined only a limited number of outcomes via self-report measures. More comprehensive evaluations, including assessments of actual behaviours (rather than intentions) and health outcomes, are necessary to determine whether there are any additional benefits or unintended consequences of the interventions. Finally, before implementing reappraisal interventions for practice, more research is needed to better evaluate the intervention (for example, via formal cost-benefit analysis and/or using the 'reach, efficacy, adoption, implementation and maintenance' framework ${ }^{106,107}$ ).

In conclusion, our findings demonstrated that two brief reappraisal interventions had robust and generalizable effects in reducing negative emotions and increasing positive emotions during the COVID-19 pandemic across countries/regions, without reducing intentions to practice preventive health behaviours. We hope this study will inform efforts to create scalable interventions for use around the world to build resilience during the pandemic and beyond.

\section{Methods}

Ethics information and participants. This study is one of three studies in the PSA COVID-19 Rapid Project. The other two studies investigated the effects of loss and gain message framing and self-determination theory-guided message framing, respectively. The other two studies are reported elsewhere. The study was conducted online, and participants clicked a single data collection link that led to either the current study or the other two studies in the COVID-19 Rapid Project. A comprehensive summary of the PSA COVID-19 Rapid Project-including descriptions of the study selection procedure, the other selected studies, the internal peer review process, and implementation plans-can be found at https:// psyarxiv.com/x976j/.

Participants were recruited by the PSA network. The PSA recruited 186 member laboratories from 55 countries/regions speaking 42 languages. Of the
27,989 participants recruited to complete the current study (not counting participants for the other two studies in the PSA COVID-19 Rapid Project), 4,050 were recruited through semi-representative panelling (on the basis of sex, age and sometimes ethnicity) from the following countries/regions: Egypt, Kenya, Nigeria, South Africa, Mexico, United States, Austria, Romania, Russia, Sweden, Switzerland, United Kingdom, China, Japan and South Korea (270 participants per country/region). The remaining participants were recruited through the research groups by convenience sampling. Each research group obtained approval from their local Ethics Committee or IRB to conduct the study, explicitly indicated that their institution did not require approval for the researchers to conduct this type of task, or explicitly indicated that the current study was covered by a pre-existing approval. Although the specifics of the consent procedure differed across research groups, all participants provided informed consent. The style and the amount of compensation varied with local conventions (a common practice in PSA). More information regarding participant compensation and sample size can be found at https://psyarxiv.com/x976j/.

\section{Procedure. An overview of the experiment is depicted in Fig. 2.}

Pre-measure. Before reading the instructions, participants reported emotions they felt in the moment (details for all study measures are described in the next section) These ratings constituted a baseline emotional measure.

Randomization to condition. Following the pre-measure, participants were randomly assigned to one of four between-subjects experimental conditions: two reappraisal intervention conditions (reconstrual and repurposing), one active control condition and one passive control condition. Because the study was conducted online, data collection was performed blind to the conditions of the participants. The content of the instructions in each condition differed, but the lengths were matched except for the passive control condition, which had a shorter set of instructions.

Participants in the two reappraisal intervention conditions (reconstrual and repurposing) and the active control condition received the following instructions: "In this study, we will show you photographs related to COVID-19 from various news sources. Our goal is to better understand how people respond to such photos, which may include feelings of fear, anger, and sadness. Sometimes emotions like these are helpful. At other times, however, these emotions can be unhelpful to us. Researchers have found that when people think their emotions are unhelpful, they can take steps to influence their emotions."

In the reconstrual condition, participants were told that (emphasis in original) "One strategy that some people find helpful for influencing their emotions is rethinking. This strategy involves changing one's thinking in order to change one's emotions. This strategy is based on the insight that different ways of interpreting or thinking about any situation can lead to different emotions. This means that finding new ways of thinking about a situation can change how you feel about the situation. For example, consider someone who stays at home under lockdown due to COVID-19 and is feeling anxious, sad, or angry. In this case, rethinking might involve realizing that the situation is only temporary because dedicated people across the world are working hard to find a vaccine." Participants were then given four examples of how rethinking might be employed for the COVID-19 situation (Example 1: "I know from world history that keeping calm and carrying on gets us through tough times."; Example 2: "Scientists across the world are working hard to find treatment and vaccines. Throughout history, humans have been resourceful in finding solutions to new challenges."; Example 3: "Washing hands, avoiding touching my face, keeping a safe distance... There are simple and effective things I can do to protect myself and my loved ones from getting sick and to stop the spread of the virus."; Example 4: "In the past, people have overcome many challenges that seemed overwhelming at the time, and we will overcome COVID-19 related challenges too.").

In the repurposing condition, participants were told that (emphasis in original) "One strategy that some people find helpful for influencing their emotions is refocusing. This strategy involves changing one's thinking in order to change one's emotions. This strategy is based on the insight that finding something good in even the most challenging situations can lead to different emotional responses. This means that refocusing on whatever good aspects may be found in a situation can change how you feel about the situation. For example, consider someone who stays at home under lockdown due to COVID-19 and is feeling anxious, sad, or angry. In this case, refocusing might involve realizing that staying at home gives them time to do things that they may not have been able to do before, like reading, painting, and spending time with family." Participants were then given four examples of how refocusing might be employed for the COVID-19 situation (Example 1: "This situation is helping us realize the importance of meaningful social connections, and helping us understand who the most important people in our lives are."; Example 2: "Medical systems are now learning to deal with amazing challenges, which will make them much more resilient in the future."; Example 3: "Even though we are physically apart, we are finding creative ways to stay connected and our hearts are more connected than ever."; Example 4: "I have been inspired by the way that frontline health care workers have responded with resilience, generosity, determination, and deep commitment.").

In the active control condition, participants were asked to reflect on their emotions as they unfold. This condition is inspired by the literature on expressive 
writing and experimental disclosure, which shows that asking people to reflect about their very deepest thoughts and feelings can improve psychological health ${ }^{77,78}$. By having an active control condition, which was likely to lead to some benefit to participants, we can make stronger inferences regarding the impact of reappraisal interventions relative to a potentially useful strategy designed to equate demand characteristics and expectancies. In the instructions, participants were told that (emphasis in original) "One strategy that some people find helpful for influencing their emotions is reflecting. This strategy involves allowing oneself to freely experience and reflect on one's thoughts and feelings. This strategy is based on the insight that reflecting on your thoughts and feelings about any situation can lead to different emotional responses. This means that exploring your thoughts and emotions can change how you feel about the situation. For example, consider someone who stays at home under lockdown due to COVID-19 and is feeling anxious, sad, or angry. In this case, reflecting might involve allowing oneself to experience these feelings and be fully immersed in the lockdown experience, reflecting on the meaning this situation has for the person and their loved ones." Participants were then given four examples of how reflecting might be employed for the COVID-19 situation (Example 1: "This situation is changing so fast, and I don't know how the future will develop."; Example 2: "People are struggling to cope with these unprecedented and overwhelming challenges."; Example 3: "Someone I love might get sick and there might not even be ventilators to help them."; Example 4: "I really wish we could find a vaccine soon.").

To reinforce what they had learned, participants in the two reappraisal conditions and the active control condition were then asked to summarize, in one or two sentences, the strategy they had just learned. This text response was collected only for exploratory purposes and was not used in confirmatory analysis.

In the passive control condition, participants received the following instructions: "In this study, we will show you photographs related to COVID-19 from various news sources. Our goal is to better understand how people respond to such photos, which may include feelings of fear, anger, and sadness. As you view these photographs, please respond as you naturally would." Having a passive control condition allowed us to have clear interpretations in the case that we found no significant difference in our contrast between both the reappraisal conditions combined and both the control conditions combined. If this was the case, we would have compared each reappraisal condition against the passive control condition and compared the active control condition against the passive control condition in the exploratory analysis to determine whether each strategy had a non-zero impact relative to individuals' natural responses.

Practice trials. After receiving instructions by condition, participants were asked to practice the strategy in two trials designed to facilitate their understanding of the strategy. The practice trials included providing ratings and written responses to two photographs (per prior research ${ }^{108}$ ). The photographs in this study were selected by our research team from major media news sources (CNN, New York Times, The Guardian and Reuters) and present situations in Asia, Europe and North America. They were rated by our team to evoke either sadness or anxiety above the midpoint on a seven-point scale ranging from 'not at all' to 'very' and to score close to or above the midpoint on a seven-point scale ranging from 'not at all' to 'very' on the question "How much do you recommend using this picture?” (photographs available at https://osf.io/8bjnz/). In each practice trial, participants saw a 'negative' photo related to the COVID-19 situation (for example, an exhausted doctor or medical workers in hazmat suits) and a reminder above the photo to use the strategy that was presented to them. In the reconstrual condition, the reminder was "As you view the photo, draw on the examples we gave you earlier in order to interpret the situation in a new way." In the repurposing condition, the reminder was "As you view the photo, draw on the examples we gave you earlier in order to focus on any good you can find in the situation." In the active control condition, the reminder was "As you view the photo, draw on the examples we gave you earlier in order to reflect on your thoughts and feelings." In the passive control condition, the reminder was "As you view the photo, respond as you naturally would." After $10 \mathrm{~s}$, participants were asked to rate their emotions in response to the photo using two corresponding unipolar five-point Likert scales, one for negative emotion and one for positive emotion. These ratings were designed to familiarize participants with the task, and were not used in the confirmatory analyses. After each photo, participants in the two reappraisal conditions and the active control condition were asked to write (in text) how they applied the strategy while observing the photo. Participants in the passive control condition were asked to write (in text) anything that comes naturally to their mind about the photo. The text response was also collected only for exploratory purposes and was not used in the confirmatory analysis. Participants in the two reappraisal conditions and the active control condition were then given one example of how the photo might be viewed (examples varied by condition). Note that the two reappraisal conditions and the active control condition were designed to be matched for demand characteristics and expectancy.

Experimental trials. Following the two practice trials, participants viewed additional photos related to the COVID-19 situation in ten experimental trials. Participants in the two reappraisal conditions and the active control condition were asked to use the strategy that they practiced, and participants in the passive control condition were asked to respond naturally. All participants saw exactly the same ten photos, but the order of the presentation was randomized across the ten experimental trials. Each photo was presented to participants with the same reminder used in the practice trials. After observing each photo for ten seconds, participants were asked to rate both their negative and positive emotions in response to the photo using the same five-point Likert scales from the practice trials.

Post-measures. In the final section of the study, participants completed several measures, including (1) negative and positive state emotions, (2) negative and positive emotions about the COVID-19 situation, (3) negative and positive anticipated emotions, (4) behavioural intentions, (5) motivation/beliefs, and (6) manipulation check.

Measures. Demographics. At the beginning of the study, participants completed a general survey that included demographic questions and some questions related to COVID-19 shared by all three studies in the PSA COVID-19 Rapid Project. Details about the general survey can be found at https://osf.io/7axc4/. While we originally planned for the general survey to appear at the end of the study, it was necessary for recruitment purposes (selecting representative panels) that it appear at the beginning of the study.

Baseline emotions. To assess baseline emotion, we asked participants how they were feeling right now at the beginning of the session on a five-point scale ranging from 1 (not at all) to 5 (extremely) (all response options were labelled and numbers were not displayed to participants for clarity). For negative baseline emotions, we measured five items on fear, anger, sadness, distrust and stress from the modified differential emotions scale ${ }^{109}$. For positive baseline emotions, we measured five items on hope, gratitude, love, inspiration and serenity from the modified differential emotions scale ${ }^{109}$ (details for all scoring rules are described in 'Analysis plan'). We also measured three items on loneliness ${ }^{110}$ and three items on social connectedness ${ }^{111}$. These six items also were included in the assessment of post-photo state emotions and in the assessment of anticipated emotions (at each assessment point, these six items were used in exploratory analyses).

Negative emotional responses. In order to capture descriptively rich, nuanced data, we measured negative emotional responses in four ways. The first way is to measure negative emotions in response to the photos. For each photo, we asked participants how negative the photo made them feel using a unipolar scale ranging from 1 (not at all) to 5 (extremely). The second way is to measure negative state emotions after viewing all ten photos. We asked participants "how you are feeling right now" with the same set of items used to measure baseline emotions, which included five negative state emotions of fear, anger, sadness, distrust and stress. The third way is to measure negative emotions about the COVID-19 situation. We asked participants how negative/hopeless they were feeling about the COVID-19 situation right now on a unipolar scale ranging from 1 (not at all) to 5 (extremely). The fourth way is to measure negative anticipated emotions, which were an exploratory outcome. We asked participants "In the next week, to what extent, if at all, do you think you will feel each of the following?" with the same set of items used to measure baseline emotions, which included five negative anticipated emotions of fear, anger, sadness, distrust and stress.

Positive emotional responses. Following a parallel procedure, we measured positive emotional responses in four ways. The first way is to measure positive emotions in response to the photos. For each photo, we asked participants how positive the photo made them feel using a unipolar scale ranging from 1 (not at all) to 5 (extremely). The second way is to measure positive state emotions after viewing all ten photos. We asked participants "how you are feeling right now" with the same set of items used to measure baseline emotions, which included five positive state emotions of hope, gratitude, love, inspiration, and serenity. The third way is to measure positive emotions about the COVID-19 situation. We asked participants how positive/hopeful they were feeling about the COVID-19 situation right now on a unipolar scale ranging from 1 (not at all) to 5 (extremely). The fourth way is to measure positive anticipated emotions, which were an exploratory outcome. We asked participants "In the next week, to what extent, if at all, do you think you will feel each of the following?" with the same set of items used to measure baseline emotions, which included five positive anticipated emotions of hope, gratitude, love, inspiration and serenity.

Behavioural intentions. In addition to the emotional responses that are central to our four confirmatory hypotheses in this study, we also examined exploratory outcomes concerning behavioural intentions. Such intentions matter because they have been shown to predict actual behaviours ${ }^{112,113}$. Following protocols from Fishbein and Ajzen ${ }^{114}$, we asked participants to indicate on a 7-point scale ranging from 1 (extremely unlikely) to 7 (extremely likely) their intentions to engage in each of 10 different behaviours within the next week. Five of the items concern potentially harmful behaviour, which we chose based on documented links between negative emotions and substance use, aggressive behaviour and excessive information seeking ${ }^{17,25,115}$. Items included: drinking too much alcohol, using too much tobacco (for example, smoking or vaping) or other recreational drugs, yelling at someone, taking anger out online and spending too much time on media. 
The other five items concerned beneficial behaviour, which we chose based on evidence that positive emotions contribute to more health behaviours ${ }^{84,85}$. Items include: eating healthy food, getting enough physical activity, practicing healthy sleep habits (for example, going to bed and waking at regular hours), washing hands regularly for at least $20 \mathrm{~s}$, and following a stay-at-home order stringently (if there isn't an order in your region now, assume that one is imposed)

Motivation and beliefs. We measured both the motivation to use the emotion regulatory strategy and the belief in the effectiveness of the emotion regulatory strategy as exploratory moderators ${ }^{71,87}$. We asked "Recall the instructions we gave you for viewing the photos. To what extent, if at all, do you agree or disagree with the following statements?" Motivation to use the emotion regulatory strategy was measured with the item: "I tried my hardest to follow the instructions I was given while viewing the photos." Belief in the effectiveness of the emotion regulatory strategy employed by participants was measured with the item "I believed that following the instructions would influence my emotions." Participants rated their answers using a 7-point scale ranging from 1 (strongly disagree) to 7 (strongly agree)

Manipulation check. We planned to evaluate participants' attention to our instructions and photos using two multiple-choice questions. The first question asked participants to choose the instructions they had at the beginning of the survey from among four options. The second question asked participants to choose the photo that was not shown to them in the survey from among three options.

For exploratory purposes, we also asked how often participants actually used each approach when viewing the photographs and their global change of emotions compared to the beginning of the study. Participants were asked, "When viewing the ten photographs related to COVID-19 earlier, how often did you use each of the following approaches?" and rated four approaches: "responding as I naturally would," "reflecting on my thoughts and feelings," "interpreting the situation in a new way," and "focusing on any good I could find in the situation." Participants rated their answers using a 5-point scale ranging from 1 (never) to 5 (always). To measure global change of emotion, participants were asked, "Overall, compared to the beginning of this study, how negative do you feel right now?" using a 5 -point scale ranging from 1 (much more negative) to 5 (much less negative) and "Overall, compared to the beginning of this study, how positive do you feel right now?" using a 5-point scale ranging from 1 (much more positive) to 5 (much less positive).

Order of items. For measures above, items belonging to the negative category (that is, negative emotional responses and intentions for harmful behaviour) and to the positive category (that is, positive emotional responses and intentions for beneficial behaviour) were presented in a counterbalanced order within each measure across participants. In other words, half of the participants always rated an item from the negative category first and then an item from the positive category, whereas the other half always rated an item from the positive category first and then an item from the negative category. For measures that have multiple items, items belonging to the negative category were randomized within the negative category, and items belonging to the positive category were randomized within the positive category. When the same set of items used to measure baseline emotions was repeated, the set had the same order for every given participant.

Analysis plan. Pre-processing. Exclusion. We planned to exclude (1) participants who answered both multiple-choice manipulation check questions incorrectly, and (2) participants who completed fewer than $50 \%$ of the questions in the study.

Reliability of measures. For items from the modified differential emotions scale ${ }^{109}$, we planned to create overall negative emotion scores at each time point by averaging the five negative emotions (fear, anger, sadness, distrust and stress) and overall positive emotion scores at each time point by averaging the five positive emotions (hope, gratitude, love, inspiration and serenity) if the average inter-item correlation was above 0.40 for negative emotions and for positive emotions, respectively. If the average inter-item correlation was below 0.40 , we would conduct an exploratory factor analysis with oblique rotation and maintain factors with an eigenvalue above 1.00. If no factors had an eigenvalue above 1 , we would report results by item rather than as a composite. The actual average inter-item correlation was 0.50 for negative baseline emotions and 0.48 for positive baseline emotions. Therefore, we created overall negative emotion scores at each time point by averaging the five negative emotions and overall positive emotion scores at each time point by averaging the five positive emotions.

Missing data. We dropped incomplete cases on an analysis-by-analysis basis. Given

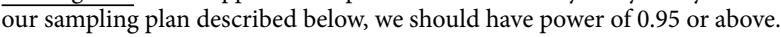

Outliers. In order to be maximally conservative, we did not define or identify outliers.

Analytic plan for hypotheses. Since negative emotional responses and positive emotional responses are separable $\mathrm{e}^{79,80}$, we examined negative emotional responses and positive emotional responses separately. To control family-wise error rates in multiple comparisons, we used the Holm-Bonferroni method within each of the four hypotheses separately. For all analyses testing negative emotional responses (hypothesis 1 and hypothesis 3), we planned to control for the participants' negative baseline emotions. As originally intended by the scale ${ }^{109}$, we planned to create an overall negative baseline emotion score by averaging the five negative emotions (fear, anger, sadness, distrust and stress). For all analyses testing positive emotional responses (hypothesis 2 and hypothesis 4), we planned to control for the participants' positive baseline emotions. As originally intended by the scale ${ }^{109}$, we planned to create an overall positive baseline emotion score by averaging the five positive emotions (hope, gratitude, love, inspiration and serenity). To account for the nested structure in our data (for example, participant nested by country/ region), we fitted multilevel models with the condition using the contrast in Table 1 , random by-country/region slopes, and random by-country/region intercepts. If a model failed to converge, we planned to explore other reasonable models ${ }^{11}$ and report results of all explored models in an appendix. We visually assessed assumptions of heteroscedasticity and normality of residuals and found no severe deviations. All tests were two-tailed.

Although we used the frequentist approach for confirmatory analyses, we also reported Bayes factors for every result to gain information about the strength of evidence provided by the data comparing the null and alternative hypotheses ${ }^{116}$. If we obtained non-significant results from the frequentist approach, we used Bayes factors to help us interpret non-significant results and differentiate between insensitive results and those that reveal good enough evidence supporting the null hypothesis. We set these evidence thresholds to $\mathrm{BF}_{10}>10$ for $\mathrm{H}_{1}$ and $\mathrm{BF}_{10}<0.1$ for $\mathrm{H}_{0}$. If Bayes factors did not cross the evidence thresholds, we think our sample size is sufficiently large that inconclusive results at this sample size would be an important message for the field. We used informed priors for the alternative model: a one-tailed Cauchy distribution with a mode of zero and a scale $r=0.18$ (hypotheses 1 and 2), $r=0.17$ (hypothesis 3 ) and $r=0.25$ (hypothesis 4 ) on the standardized effect size. These priors were based on the lowest available estimates of effect sizes in past research (more information in 'Sampling plan'). At stage 1, we wrote the code for the Bayesian part of our analysis plan using the BayesFactor package $^{117}$ in R. We also planned to investigate the sensitivity of our conclusions to priors using robustness regions ${ }^{118}$, which involves calculating a Bayes factor under a large number of different priors to see how the Bayes factor changes. After we collected our data, we made the following adjustments to our plans for our Bayesian analysis. First, to estimate the Bayesian models, we switched from the BayesFactor package to the brms package ${ }^{119}$ because of its superior handling of random effects. Our brms models used four chains, each with 1,000 warm-up samples, 10,000 post-warm-up samples and a thinning rate of 1 . To calculate Bayes factors, we used bridge sampling, as implemented in the bayestest $\mathrm{R}^{120}$ and bridgesampling ${ }^{121}$ packages, to compare the marginal likelihoods of the full model versus a null model that does not contain one of our two focal contrasts. Second, we discovered that the Bayesian versions of our models involving emotional responses to the photos had high computational requirements due to the inclusion of two sources of random effect (country/region and participant) rather than one. To make these models more computationally manageable we simplified the dataset by computing the average emotional response to each photo for each participant and using this as the outcome variable. This allowed us to omit the by-participant random effect in these models and drastically reduce the resource requirements and compute time. Although these simplified models do not separate participant-specific variance from error variance, our analysis plan had no plans to interpret these sources of variation separately, so we reasoned this simplification was a fair way to obtain the same mathematical results as required by our analysis plan at a lower computational cost. Finally, we simplified the robustness analyses by only investigating how the Bayes factors change with one very large prior $(r=1.0)$ rather than computing full robustness regions. We made this last change to once again reduce the compute time to manageable levels. If the Bayes factors under the large prior are in line with those generated by the pre-registered priors (which are already very small), the results should be robust to other reasonable priors.

Tests for hypotheses 1 and 3. Overall, we expected that reappraisal interventions (versus control) would reduce negative emotional responses (hypothesis 1), and that reconstrual would lead to greater decreases in negative emotional responses than repurposing (hypothesis 3). We tested hypothesis 1 and hypothesis 3 using two orthogonal contrasts (Table 1). The first contrast is between both reappraisal conditions combined and both control conditions combined for hypothesis 1 . The second contrast is between the reconstrual condition and the repurposing condition for hypothesis 3 . Negative emotional responses were measured in four ways (negative emotions in response to the photos, negative state emotions after viewing the photos, negative emotions about the COVID-19 situation, and negative anticipated emotions). We had confirmatory hypotheses regarding the first three outcomes and examined negative anticipated emotions in the exploratory analysis. Therefore, hypothesis 1 can be subdivided into hypotheses 1a to 1c, and hypothesis 3 can be subdivided into hypotheses 3 a to $3 \mathrm{c}$. We planned to consider a hypothesis to be supported if at least 1 of the 3 sub-hypotheses is significant after HolmBonferroni correction (controlling for 3 comparisons within each hypothesis). If we found non-significant results for any sub-hypothesis, we compared each reappraisal condition against the passive control condition and compared the active control condition against the passive control condition in the exploratory analysis 
to determine whether each strategy had a non-zero impact relative to individuals' natural responses.

Testing effects on negative emotions in response to the photos. We expected that reappraisal interventions (versus control) would reduce negative emotions in response to the photos (hypothesis 1a), and reconstrual would lead to greater decreases in negative emotional responses in response to the photos than repurposing (hypothesis $3 \mathrm{a}$ ). We modelled ratings of negativity in response to each photo in the experimental trials as a function of the fixed effects of condition using our contrast. We included by-participant random intercepts, by-country/region random intercepts, as well as by-country/region random slopes for each contrast.

Testing effects on negative state emotions. We expected that reappraisal interventions (versus control) would reduce negative state emotions (hypothesis 1b) and reconstrual would lead to greater decreases in negative state emotions than repurposing (hypothesis $3 \mathrm{~b}$ ). Similar to creating the overall negative baseline emotion score, we planned to create an overall negative state emotion score by averaging the five negative emotions (fear, anger, sadness, distrust and stress). We modelled the overall negative state emotion score as a function of the fixed effects of condition using our contrast. We included by-country/region random intercepts, as well as by-country/region random slopes for each contrast.

Testing effects on negative emotions about the COVID-19 situation. We expected that reappraisal interventions (versus control) would reduce negative emotions about the COVID-19 situation (hypothesis 1c), and reconstrual would lead to greater decreases in negative emotions about the COVID-19 situation than repurposing (hypothesis $3 \mathrm{c}$ ). We modelled negative emotions about the COVID-19 situation as a function of the fixed effects of condition using our contrast. We included by-country/region random intercepts, as well as by-country/region random slopes for each contrast.

Tests for hypotheses 2 and 4. Overall, we expected that reappraisal interventions (versus control) would increase positive emotional responses (hypothesis 2), and repurposing would lead to greater increases in positive emotional responses than reconstrual (hypothesis 4). We tested hypothesis 2 and hypothesis 4 using two orthogonal contrasts (Table 1). The first contrast is between both reappraisal conditions combined and both control conditions combined for hypothesis 2 . The second contrast is between the reconstrual condition and the repurposing condition for hypothesis 4 . Positive emotional responses were measured in four ways (positive emotions in response to the photos, positive state emotions after viewing the photos, positive emotions about the COVID-19 situation, and positive anticipated emotions). We had confirmatory hypotheses regarding the first three outcomes and examined positive anticipated emotions in an exploratory analysis. Therefore, hypothesis 2 can be subdivided into hypotheses 2 a to $2 c$, and hypothesis $4 \mathrm{can}$ be subdivided into hypotheses $4 \mathrm{a}$ to $4 \mathrm{c}$. We planned to consider a hypothesis to be supported if at least 1 of the 3 sub-hypotheses is significant after HolmBonferroni correction (controlling for 3 comparisons within each hypothesis). If we found non-significant results for any sub-hypothesis, we would compare each reappraisal condition against the passive control condition and compare the active control condition against the passive control condition in the exploratory analysis to determine whether each strategy had a non-zero impact relative to individuals' natural responses.

Testing effects on positive emotions in response to the photos. We expected that reappraisal interventions (versus control) would increase positive emotions in response to the photos (hypothesis $2 \mathrm{a}$ ), and that repurposing would lead to greater increases in positive emotions in response to the photos than reconstrual (hypothesis 4a). We modelled ratings of positivity in response to each photo in the experimental trials as a function of the fixed effects of condition using our contrast. We included by-participant random intercepts, by-country/region random intercepts, as well as by-country/region random slopes for each contrast.

Testing effects on positive state emotions. We expected that reappraisal interventions (versus control) would increase positive state emotions (hypothesis $2 \mathrm{~b}$ ), and repurposing would lead to greater increases in positive state emotions in response to the photos than reconstrual (hypothesis $4 \mathrm{~b}$ ). Similar to creating the overall positive baseline emotion score, we planned to create an overall positive state emotion score by averaging the five positive emotions (hope, gratitude, love, inspiration and serenity). We modelled the overall positive state emotion score as a function of the fixed effects of condition using our contrast. We planned to include by-country/region random intercepts, as well as by-country/region random slopes for each contrast. However, the model could not converge when we included by-country/region random slopes for contrast 2 . To make the model converge, we did not include by-country/region random slopes for contrast 2.

Testing effects on positive emotions about the COVID-19 situation. We expected that reappraisal interventions (versus control) would increase positive emotions about the COVID-19 situation (hypothesis 2c), and repurposing would lead to greater increases in positive emotions about the COVID-19 situation than reconstrual (hypothesis 4c). We modelled positive emotions about the COVID-19 situation as a function of the fixed effects of condition using our contrast. We included by-country/region random intercepts, as well as by-country/region random slopes for each contrast.

Exploratory analyses. We conducted a series of exploratory analyses to address supplemental questions regarding our hypotheses, including, but not limited to: (1) Were there any differences in other pairwise comparisons in testing hypotheses $1-2$ ? (2) Were there emotion-specific effects of reappraisal ${ }^{122}$ ? (3) Were the effects on emotions subjectively detectable by participants ${ }^{123}$ ? Did the effects of strategy use vary by (4) motivation to use the strategy ${ }^{71} ;(5)$ beliefs in the strategy's effectiveness ${ }^{87}$; or (6) the participant's country of residence ${ }^{90}$ ?

We investigated the impacts of strategy use on other outcomes, including, but not limited to: (1) positive and negative anticipated emotions; (2) intentions to enact potentially harmful versus beneficial behaviours (results in Supplementary Table 14); and (3) loneliness and social connectedness (results in Supplementary Table 15).

Sampling plan. Expected effect sizes. In order to compare effect sizes across studies, below we report values of Cohen's $d$, which in some cases were transformed or calculated from the results reported in the original studies (see Supplementary Table 16 for details). Several caveats are in order regarding the effect sizes that follow. First, meta-analyses tend to overestimate effect sizes, although the size of overestimation varies considerably across studies and sometimes shows no overestimation ${ }^{124}$. Second, most previous studies were conducted in the laboratory, whereas the current study was conducted online. Third, the current crisis is likely to lead to strong emotional responses, especially for participants who are facing financial or health-related setbacks, although strong negative emotions also motivate people to regulate emotions more ${ }^{64}$. These caveats suggest uncertainty in effect sizes.

In general, reappraisal has an average effect size of $d=0.45,95 \% \mathrm{CI}=[0.35$, $0.56]$ in changing emotion experience relative to passive control conditions (that is, no instruction, instructions to experience naturally, instructions to not regulate in a certain manner, or instructions to enhance or maintain the focal emotion) (a meta-analysis ${ }^{39}$ finds no evidence of publication bias). Experimental disclosure and expressive writing, which inspired the instruction in the active control condition, have an average effect size of $d=0.07,95 \% \mathrm{CI}=[0.05,0.17]$ in improving psychological health (including emotional responses), relative to engaging in non-treatment neutral activities (for example, describing what they have done in the past $24 \mathrm{~h}$ ) or no activities (a meta-analysis ${ }^{77}$ finds evidence of publication bias). These works suggest the lowest available estimate of the effect size to be $d=0.18$ (subtracting the upper bound of $95 \%$ CI $d=0.17$ for experimental disclosure and expressive writing from the lower bound of $95 \% \mathrm{CI}$ of $d=0.35$ for the reappraisal interventions) between our reappraisal interventions and the control conditions for hypothesis 1 and hypothesis 2 .

In relation to the comparison between reconstrual and repurposing, although prior research has not used the same theoretical framework ${ }^{76}$ to empirically contrast reconstrual and repurposing as we did in the current study, research on closely related constructs can provide estimates of effect sizes. Reconstrual is most similar to a previously studied subtype of reappraisal called 'reappraising emotional stimulus' in Webb, Miles and Sheeran's meta-analysis ${ }^{39}$, which has a $d=0.38,95 \% \mathrm{CI}=[0.21,0.55]$ in changing emotion experience (this effect size is primarily for negative emotions, as all but one study examined negative emotions). Repurposing is similar to the construct 'benefit finding' (perceiving positive consequences that resulted from a traumatic event), which is associated with positive well-being, $d=0.45,95 \% \mathrm{CI}=[0.37,0.52]$, but not global distress, $d=0.00$, $95 \% \mathrm{CI}=[-0.04,0.04]$ (meta-analysis $\left.{ }^{81}\right)$. Repurposing is also similar to the subtype of reappraisal called 'positive reappraisal', which is more effective in increasing positive thoughts than other types of reappraisals, $d=0.49,95 \% \mathrm{CI}=[0.25,0.72]$ relative to detached reappraisal ${ }^{125}$. These works suggest the lowest available estimate of the effect size to be $d=0.17$ (subtracting the upper bound of $95 \% \mathrm{CI}$ $d=0.04$ for the association between benefit finding and global distress from the lower bound of $95 \% \mathrm{CI}$ of $d=0.21$ for 'reappraising emotional stimulus ${ }^{39}$ between reconstrual and repurposing in changing negative emotions for hypothesis 3 ), and $d=0.25$ (the lower bound of $95 \%$ CI of positive reappraisal in increasing positive thoughts than detached reappraisal ${ }^{125}$ ) between reconstrual and repurposing in changing positive emotions for hypothesis 4 .

Sample size. For practical reasons, sample size was decided primarily on the basis of the availability of resources among members of the PSA.

Adjusted alpha levels. The tests of each hypothesis involved three comparisons, with $\alpha$ for the smallest $P$ value being 0.017 (that is, $0.05 / 3$ ), $\alpha$ for the second-smallest $P$ value being 0.025 (that is, $0.05 / 2$ ), and $\alpha$ for the largest $P$ value being 0.05 (HolmBonferroni corrections).

Power analysis. We conducted a simulation study to estimate power for a variety of potential effect sizes $(|d|=0.05$ to 0.29 , separated by increments of 0.02$)$, number of countries/regions $\left(N_{\text {country/region }}=30,35,40,45,50,55,60\right)$, within-country/ region sample sizes $(N=200,400,600,800)$, by-country/region intercept variances 
$\left(\sigma^{2}{ }_{\text {intercept }}=0.05,0.30,0.55,0.80\right)$, and by-country/region slope variances $\left(\sigma_{\text {slope }}^{2}=0.0,0.02,0.03,0.04\right)$ at $\alpha=.017$. The lowest level of intercept variances in our simulation was chosen on the basis of an ongoing multi-country/region project tracking rates of depression $\left(\sigma_{\text {intercept }}^{2}=0.04\right)$ and worries about the COVID-19 $\left(\sigma_{\text {intercept }}^{2}=0.06\right)$ across countries/regions during the COVID-19 outbreak $^{126}$ (details in Supplementary Table 16). The lowest level of slope variances in our simulation was chosen on the basis of the average slope variance $\left(\sigma_{\text {slope }}^{2}<0.01\right)$ in a large multi-site, multi-country/region project involving 28 psychological manipulations ${ }^{127}$. The slope variances capture the variability of the effect of psychological manipulations, and there is no apparent reason to expect that the effect of reappraisal interventions on emotions is more variable than most other psychological manipulations reported in Klein et al. ${ }^{127}$. In fact, appraisal theories of emotion argue that the relationship between appraisals and emotions is culturally universal ${ }^{128}$, suggesting low variability. As one example to show that similar appraisals associate with similar emotional experiences, we found the associations varied little across countries/regions between perceived insufficient government response and depression $\left(\sigma_{\text {slope }}^{2}=0.003\right)$ and between perceived insufficient government response and worries $\left(\sigma_{\text {slope }}^{2}=0.003\right)$ during the COVID-19 pandemic ${ }^{126}$ (details in Supplementary Table 16), consistent with the observation of low slope variances $\left(\sigma_{\text {slope }}^{2}<0.01\right)$ in Klein et al. ${ }^{127}$. Despite expecting low variability from empirical findings and theories, we tested a variety of intercept variances and slope variances in our power simulation, some of which were much higher than those reporrted in refs. ${ }^{127,126}$ to be maximally conservative. We conducted 1,000 simulations for each set of simulation parameters using the simr package ${ }^{129}$ using computing power harnessed through the Open Science Grid ${ }^{130,131}$

We show comprehensive results for our simulation study at https://osf.io/ $\mathrm{mf} 5 \mathrm{z} 4 /$. In our final sample after pre-registered exclusion, 37 countries/regions had over 200 participants, surpassing the $95 \%$ power criterion based on simulations.

Reporting Summary. Further information on research design is available in the Nature Research Reporting Summary linked to this article.

\section{Data availability}

The analytic dataset is available at https://osf.io/jeu73/. Materials are available at https://osf.io/4yf9d/, with additional relevant materials for the PSA's rapid-response COVID-19 projects at https://osf.io/s4hj2/.

\section{Code availability}

All analysis code (completed in R) is available at https://osf.io/jeu73/.

Received: 17 April 2020; Accepted: 28 June 2021;

Published online: 2 August 2021

\section{References}

1. New poll: COVID-19 impacting mental well-being: Americans feeling anxious, especially for loved ones; older adults are less anxious. American Psychiatric Association https://www.psychiatry.org/newsroom/news-releases/ new-poll-covid-19-impacting-mental-well-being-americans-feeling -anxious-especially-for-loved-ones-older-adults-are-less-anxious (5 May 2020).

2. Barari, S. et al. Evaluating COVID-19 public health messaging in Italy: self-reported compliance and growing mental health concerns. Preprint at medRxiv https://doi.org/10.1101/2020.03.27.20042820 (2020).

3. Hou, Z., Du, F., Jiang, H., Zhou, X. \& Lin, L. Assessment of public attention, risk perception, emotional and behavioural responses to the COVID-19 outbreak: social media surveillance in China. Preprint at SSRN https://doi.org/10.2139/ssrn.3551338 (2020).

4. Kirzinger, A., Kearney, A., Hamel, L. \& Brodie, M. KFF health tracking poll-early April 2020: the impact of coronavirus on life in America. The Henry J. Kaiser Family Foundation https://www.kff.org/health-reform/ report/kff-health-tracking-poll-early-april-2020/ (2020).

5. Langer, G. Coronavirus impacts: disrupted lives, elevated stress, and soaring worry: Poll. ABC News https://abcnews.go.com/Politics/coronavirusimpacts-disrupted-lives-elevated-stress-soaring-worry/story?id=69812058 (5 May 2020).

6. Li, S., Wang, Y., Xue, J., Zhao, N. \& Zhu, T. The impact of COVID-19 epidemic declaration on psychological consequences: a study on active Weibo users. Int. J. Environ. Res. Public Health 17, 2032 (2020).

7. McCarthy, J. Three in four Americans worry about COVID-19 exposure. Gallup.com https://news.gallup.com/poll/307646/three-four-americansworry-covid-exposure.aspx (5 May 2020).

8. Pinnington, B. Britain's view of COVID-19 as 'high threat' to their business jumps 19 points in a week. Ipsos MORI https://www.ipsos.com/ipsos-mori/ en-uk/britains-view-covid-19-high-threat-their-business-jumps-19-pointsweek (5 May 2020).

9. Smith, M. How is COVID-19 affecting British opinions, jobs and wellbeing? YouGov https://yougov.co.uk/topics/politics/articles-reports/2020/03/24/ how-covid-19-affecting-british-opinions-jobs-and-w (5 May 2020).
10. Zhao, Y., Cheng, S., Yu, X. \& Xu, H. Chinese public's attention to the COVID-19 epidemic on social media: observational descriptive study. J. Med. Internet Res. 22, e18825 (2020).

11. Jackson, C. \& Newall, M. Partisanship reemerging in American views of coronavirus. Ipsos https://www.ipsos.com/en-us/news-polls/axios-ipsoscoronavirus-index (5 May 2020).

12. Stremikis, K. In COVID-19 tracking poll, Californians report worsening mental health, cost worries. California Health Care Foundation https:// www.chcf.org/blog/covid-19-tracking-poll-californians-report-worseningmental-health-cost-worries/ (2020).

13. COVID-19 and ending violence against women and girls. UN Women https://www.unwomen.org/en/digital-library/publications/2020/04/ issue-brief-covid-19-and-ending-violence-against-women-and-girls (5 May 2020).

14. Sheppes, G., Suri, G. \& Gross, J. J. Emotion regulation and psychopathology. Annu. Rev. Clin. Psychol. 11, 379-405 (2015).

15. Cacioppo, J. T. \& Cacioppo, S. The growing problem of loneliness. Lancet 391, 426 (2018).

16. Cooper, M. L., Frone, M. R., Russell, M. \& Mudar, P. Drinking to regulate positive and negative emotions: a motivational model of alcohol use. J. Pers. Soc. Psychol. 69, 990-1005 (1995).

17. Dorison, C. A. et al. Sadness, but not all negative emotions, heightens addictive substance use. Proc. Natl Acad. Sci. USA 117, 943-949 (2020).

18. Kassel, J. D., Stroud, L. R. \& Paronis, C. A. Smoking, stress, and negative affect: correlation, causation, and context across stages of smoking. Psychol. Bull. 129, 270-304 (2003).

19. Brod, S., Rattazzi, L., Piras, G. \& D’Acquisto, F. 'As above, so below' examining the interplay between emotion and the immune system. Immunology 143, 311-318 (2014).

20. Denson, T. F., Spanovic, M. \& Miller, N. Cognitive appraisals and emotions predict cortisol and immune responses: a meta-analysis of acute laboratory social stressors and emotion inductions. Psychol. Bull. 135, 823-853 (2009).

21. Moons, W. G. \& Shields, G. S. Anxiety, not anger, induces inflammatory activity: an avoidance/approach model of immune system activation. Emotion 15, 463-476 (2015).

22. Alvaro, P. K., Roberts, R. M. \& Harris, J. K. A systematic review assessing bidirectionality between sleep disturbances, anxiety, and depression. Sleep 36, 1059-1068 (2013).

23. Adam, T. C. \& Epel, E. S. Stress, eating and the reward system. Physiol. Behav. 91, 449-458 (2007).

24. Groesz, L. M. et al. What is eating you? Stress and the drive to eat. Appetite 58, 717-721 (2012).

25. Birkley, E. L. \& Eckhardt, C. I. Anger, hostility, internalizing negative emotions, and intimate partner violence perpetration: a meta-analytic review. Clin. Psychol. Rev. 37, 40-56 (2015).

26. Roberton, T., Daffern, M. \& Bucks, R. S. Emotion regulation and aggression. Aggress. Violent Behav. 17, 72-82 (2012).

27. Foley, A. E. et al. The math anxiety-performance link: a global phenomenon. Curr. Dir. Psychol. Sci. 26, 52-58 (2017).

28. Diener, E., Thapa, S. \& Tay, L. Positive emotions at work. Annu. Rev. Organ. Psychol. Organ. Behav. 7, 451-477 (2020).

29. Shockley, K. M., Ispas, D., Rossi, M. E. \& Levine, E. L. A meta-analytic investigation of the relationship between state affect, discrete emotions, and job performance. Hum. Perform. 25, 377-411 (2012).

30. Haushofer, J. \& Fehr, E. On the psychology of poverty. Science 344, 862-867 (2014).

31. Lerner, J. S., Li, Y., Valdesolo, P. \& Kassam, K. S. Emotion and decision making. Annu. Rev. Psychol. 66, 799-823 (2015).

32. Bonanno, G. A. Loss, trauma, and human resilience: have we underestimated the human capacity to thrive after extremely aversive events? Am. Psychol. 59, 20-28 (2004).

33. Fletcher, D. \& Sarkar, M. Psychological resilience. Eur. Psychol. 18, 12-23 (2013).

34. Southwick, S. M. \& Charney, D. S. The science of resilience: implications for the prevention and treatment of depression. Science 338, 79-82 (2012).

35. McRae, K. \& Gross, J. J. Emotion regulation. Emotion 20, 1-9 (2020).

36. Kalisch, R., Müller, M. B. \& Tuescher, O. A conceptual framework for the neurobiological study of resilience. Behav. Brain Sci. 38, e92 (2014).

37. McRae, K. \& Mauss, I. B. Increasing Positive Emotion in Negative Contexts: Emotional Consequences, Neural Correlates, and Implications for Resilience. Positive Neuroscience (Oxford Univ. Press, 2016).

38. Tugade, M. M. \& Fredrickson, B. L. Regulation of positive emotions: emotion regulation strategies that promote resilience. J. Happiness Stud. 8, 311-333 (2007).

39. Webb, T. L., Miles, E. \& Sheeran, P. Dealing with feeling: a meta-analysis of the effectiveness of strategies derived from the process model of emotion regulation. Psychol. Bull. 138, 775-808 (2012).

40. Denny, B. T. \& Ochsner, K. N. Behavioral effects of longitudinal training in cognitive reappraisal. Emotion 14, 425-433 (2014). 
41. Gross, J. J. Antecedent- and response-focused emotion regulation: divergent consequences for experience, expression, and physiology. J. Pers. Soc. Psychol. 74, 224-237 (1998).

42. Jackson, D. C., Malmstadt, J. R., Larson, C. L. \& Davidson, R. J. Suppression and enhancement of emotional responses to unpleasant pictures. Psychophysiology 37, 515-522 (2000).

43. Denson, T. F., Grisham, J. R. \& Moulds, M. L. Cognitive reappraisal increases heart rate variability in response to an anger provocation. Motiv. Emot. 35, 14-22 (2011)

44. Dillon, D. G. \& LaBar, K. S. Startle modulation during conscious emotion regulation is arousal-dependent. Behav. Neurosci. 119, 1118-1124 (2005).

45. Ray, R. D., McRae, K., Ochsner, K. N. \& Gross, J. J. Cognitive reappraisal of negative affect: converging evidence from EMG and self-report. Emotion 10, 587-592 (2010).

46. Chang, L. J., Gianaros, P. J., Manuck, S. B., Krishnan, A. \& Wager, T. D. A sensitive and specific neural signature for picture-induced negative affect. PLoS Biol. 13, e1002180 (2015)

47. Dörfel, D. et al. Common and differential neural networks of emotion regulation by detachment, reinterpretation, distraction, and expressive suppression: a comparative fMRI investigation. NeuroImage 101 298-309 (2014).

48. Hajcak, G. \& Nieuwenhuis, S. Reappraisal modulates the electrocortical response to unpleasant pictures. Cogn. Affect. Behav. Neurosci. 6, 291-297 (2006)

49. Kalisch, R. et al. Anxiety reduction through detachment: subjective, physiological, and neural effects. J. Cogn. Neurosci. 17, 874-883 (2005).

50. Lohani, M. \& Isaacowitz, D. M. Age differences in managing response to sadness elicitors using attentional deployment, positive reappraisal and suppression. Cogn. Emot. 28, 678-697 (2014).

51. Ochsner, K. N., Bunge, S. A., Gross, J. J. \& Gabrieli, J. D. E. Rethinking feelings: an fmri study of the cognitive regulation of emotion. J. Cogn. Neurosci. 14, 1215-1229 (2002).

52. Schaefer, S. M. et al. Modulation of amygdalar activity by the conscious regulation of negative emotion. J. Cogn. Neurosci. 14, 913-921 (2002).

53. Shahane, A. D., Lopez, R. B. \& Denny, B. T. Implicit reappraisal as an emotional buffer: reappraisal-related neural activity moderates the relationship between inattention and perceived stress during exposure to negative stimuli. Cogn. Affect. Behav. Neurosci. 19, 355-365 (2019).

54. English, T., John, O. P., Srivastava, S. \& Gross, J. J. Emotion regulation and peer-rated social functioning: a 4-year longitudinal study. J. Res. Personal. 46, 780-784 (2012).

55. Davis, E. L. \& Levine, L. J. Emotion regulation strategies that promote learning: reappraisal enhances children's memory for educational information. Child Dev. 84, 361-374 (2013).

56. Ivcevic, Z. \& Brackett, M. Predicting school success: comparing conscientiousness, grit, and emotion regulation ability. J. Res. Personal. 52 29-36 (2014).

57. Gross, J. J. \& John, O. P. Individual differences in two emotion regulation processes: implications for affect, relationships, and well-being. J. Pers. Soc. Psychol. 85, 348-362 (2003).

58. Aldao, A., Nolen-Hoeksema, S. \& Schweizer, S. Emotion-regulation strategies across psychopathology: a meta-analytic review. Clin. Psychol. Rev. 30, 217-237 (2010).

59. Cludius, B., Mennin, D. \& Ehring, T. Emotion regulation as a transdiagnostic process. Emotion 20, 37-42 (2020).

60. Appleton, A. A., Buka, S. L., Loucks, E. B., Gilman, S. E. \& Kubzansky, L. D. Divergent associations of adaptive and maladaptive emotion regulation strategies with inflammation. Health Psychol. 32, 748-756 (2013).

61. Appleton, A. A., Loucks, E. B., Buka, S. L. \& Kubzansky, L. D. Divergent associations of antecedent- and response-focused emotion regulation strategies with midlife cardiovascular disease risk. Ann. Behav. Med. 48, 246-255 (2014).

62. Veer, I. M. et al. Psycho-social factors associated with mental resilience in the corona lockdown. Transl. Psychiatry 11, 67 (2021).

63. Suri, G., Whittaker, K. \& Gross, J. J. Launching reappraisal: it's less common than you might think. Emotion 15, 73-77 (2015)

64. Milyavsky, M. et al. To reappraise or not to reappraise? Emotion regulation choice and cognitive energetics. Emotion 19, 964-981 (2019).

65. Cohen, N. \& Ochsner, K. N. From surviving to thriving in the face of threats: the emerging science of emotion regulation training. Curr. Opin. Behav. Sci. 24, 143-155 (2018)

66. Denny, B. T. Getting better over time: a framework for examining the impact of emotion regulation training. Emotion 20, 110-114 (2020).

67. Woud, M. L., Holmes, E. A., Postma, P., Dalgleish, T. \& Mackintosh, B. Ameliorating intrusive memories of distressing experiences using computerized reappraisal training. Emotion 12, 778-784 (2012).

68. Goldin, P. R. et al. Cognitive reappraisal self-efficacy mediates the effects of individual cognitive-behavioral therapy for social anxiety disorder. J. Consult. Clin. Psychol. 80, 1034-1040 (2012).
69. Kivity, Y. \& Huppert, J. D. Does cognitive reappraisal reduce anxiety? A daily diary study of a micro-intervention with individuals with high social anxiety. J. Consult. Clin. Psychol. 84, 269-283 (2016).

70. Denny, B. T., Inhoff, M. C., Zerubavel, N., Davachi, L. \& Ochsner, K. N Getting over it: long-lasting effects of emotion regulation on amygdala response. Psychol. Sci. 26, 1377-1388 (2015).

71. Tamir, M., Halperin, E., Porat, R., Bigman, Y. E. \& Hasson, Y. When there's a will, there's a way: disentangling the effects of goals and means in emotion regulation. J. Pers. Soc. Psychol. 116, 795-816 (2019).

72. Porat, R., Halperin, E. \& Tamir, M. What we want is what we get: group-based emotional preferences and conflict resolution. J. Pers. Soc. Psychol. 110, 167-190 (2016)

73. Hurtado-Parrado, C. et al. Emotion regulation and attitudes toward conflict in Colombia: effects of reappraisal training on negative emotions and support for conciliatory and aggressive statements. Front. Psychol. 10, 908 (2019).

74. Halperin, E., Porat, R., Tamir, M. \& Gross, J. J. Can emotion regulation change political attitudes in intractable conflicts? From the laboratory to the field. Psychol. Sci. 24, 106-111 (2012).

75. Moshontz, H. et al. The Psychological Science Accelerator: advancing psychology through a distributed collaborative network. Adv. Methods Pract. Psychol. Sci. 1, 501-515 (2018).

76. Uusberg, A., Taxer, J. L., Yih, J., Uusberg, H. \& Gross, J. J. Reappraising reappraisal. Emot. Rev. 11, 267-282 (2019).

77. Frattaroli, J. Experimental disclosure and its moderators: a meta-analysis. Psychol. Bull. 132, 823-865 (2006).

78. Pennebaker, J. W. \& Smyth, J. M. Opening Up by Writing It Down: How Expressive Writing Improves Health and Eases Emotional Pain 3rd edn (Guilford Publications, 2016).

79. Kreibig, S. D. \& Gross, J. J. Understanding mixed emotions: paradigms and measures. Curr. Opin. Behav. Sci. 15, 62-71 (2017).

80. Larsen, J. T. \& McGraw, A. P. Further evidence for mixed emotions. J. Pers. Soc. Psychol. 100, 1095-1110 (2011).

81. Helgeson, V. S., Reynolds, K. A. \& Tomich, P. L. A meta-analytic review of benefit finding and growth. J. Consult. Clin. Psychol. 74, 797-816 (2006).

82. Shiota, M. N. Silver linings and candles in the dark: differences among positive coping strategies in predicting subjective well-being. Emotion 6, 335-339 (2006).

83. Kok, G., Peters, G.-J. Y., Kessels, L. T. E., ten Hoor, G. A. \& Ruiter, R. A. C. Ignoring theory and misinterpreting evidence: the false belief in fear appeals. Health Psychol. Rev. 12, 111-125 (2018)

84. Fredrickson, B. L. \& Joiner, T. Reflections on positive emotions and upward spirals. Perspect. Psychol. Sci. 13, 194-199 (2018).

85. Pressman, S. D., Jenkins, B. N. \& Moskowitz, J. T. Positive affect and health what do we know and where next should we go? Annu. Rev. Psychol. 70, 627-650 (2019).

86. Ogedegbe, G. O. et al. A randomized controlled trial of positive-affect intervention and medication adherence in hypertensive African Americans. Arch. Intern. Med. 172, 322-326 (2012).

87. Bigman, Y. E., Mauss, I. B., Gross, J. J. \& Tamir, M. Yes I can: expected success promotes actual success in emotion regulation. Cogn. Emot. 30, 1380-1387 (2016)

88. Hittner, E. F., Rim, K. L. \& Haase, C. M. Socioeconomic status as a moderator of the link between reappraisal and anxiety: laboratory-based and longitudinal evidence. Emotion 19, 1478-1489 (2019).

89. Troy, A. S., Ford, B. Q., McRae, K., Zarolia, P. \& Mauss, I. Change the things you can: emotion regulation is more beneficial for people from lower than from higher socioeconomic status. Emotion 17, 141-154 (2017).

90. Miyamoto, Y., Ma, X. \& Petermann, A. G. Cultural differences in hedonic emotion regulation after a negative event. Emotion 14, 804-815 (2014).

91. Hofstede, G., Hofstede, G. J. \& Minkov, M. Cultures and Organizations: Software of the Mind 3rd edn, (McGraw-Hill, New York, 2010).

92. Nakagawa, S. \& Schielzeth, H. A general and simple method for obtaining R2 from generalized linear mixed-effects models. Methods Ecol. Evol. 4, 133-142 (2013)

93. Eid, M. \& Diener, E. Norms for experiencing emotions in different cultures: inter- and intranational differences. J. Pers. Soc. Psychol. 81, 869-885 (2001).

94. Ferrer, R., Klein, W., Lerner, J. S., Reyna, V. F. \& Keltner, D. in Behavioral Economics and Public Health (eds Roberto, C. \& Kawachi, I.) (Harvard Univ. Press, 2016).

95. Smith, A. M. et al. Coping with health threats: the costs and benefits of managing emotions. Psychol. Sci. https://doi.org/10.1177/ 09567976211024260 (2021).

96. Pfattheicher, S., Nockur, L., Böhm, R., Sassenrath, C. \& Petersen, M. B. The emotional path to action: empathy promotes physical distancing and wearing of face masks during the COVID-19 pandemic. Psychol. Sci. 31, 1363-1373 (2020)

97. Jørgensen, F., Bor, A. \& Petersen, M. B. Compliance without fear: individual-level protective behaviour during the first wave of the COVID-19 pandemic. Br. J. Health Psychol. 26, 679-696 (2021). 
98. Jordan, J., Yoeli, E. \& Rand, D. Don't get it or don't spread it? Comparing self-interested versus prosocial motivations for COVID-19 prevention behaviors. Preprint at PsyArXiv https://doi.org/10.31234/osf.io/yuq7x (2020).

99. Willroth, E. C. et al. A test of resilience theory in the context of a global crisis. Preprint at PsyArXiv https://doi.org/10.31234/osf.io/g3jr7 (2021).

100. Madison, A. A., Shrout, M. R., Renna, M. E. \& Kiecolt-Glaser, J. K. Psychological and behavioral predictors of vaccine efficacy: considerations for COVID-19. Perspect. Psychol. Sci. 16, 191-203 (2021).

101. Ferrer, R. A. et al. The role of incidental affective states in appetitive risk behavior: a meta-analysis. Health Psychol. 39, 1109-1124 (2020).

102. Kemp, E., Cowart, K. \& Bui, M. Promoting consumer well-being: examining emotion regulation strategies in social advertising messages. J. Bus. Res. 112, 200-209 (2020).

103. Porat, R., Erel, L., Pnueli, V. \& Halperin, E. Developing ReApp: an emotion regulation mobile intervention for intergroup conflict. Cogn. Emot. 34, 1326-1342 (2020).

104. Keltner, D. \& Lerner, J. S. in Handbook of Social Psychology (eds Fiske, S. T. et al.) (John Wiley \& Sons, 2010).

105. Henrich, J., Heine, S. J. \& Norenzayan, A. The weirdest people in the world. Behav. Brain Sci. 33, 61-83 (2010).

106. Glasgow, R. E., Vogt, T. M. \& Boles, S. M. Evaluating the public health impact of health promotion interventions: the RE-AIM framework. Am. J. Public Health 89, 1322-1327 (1999).

107. Premachandra, B. \& Lewis, N. A. J. Do we report the information that is necessary to give psychology away? A scoping review of the psychological intervention literature 2000-2018. Perspect. Psychol. Sci. https://doi.org/ $10.1177 / 1745691620974774$ (2021).

108. Goldin, P. R. \& Gross, J. J. Effects of mindfulness-based stress reduction (MBSR) on emotion regulation in social anxiety disorder. Emotion 10, 83-91 (2010).

109. Fredrickson, B. L. in Advances in Experimental Social Psychology Volume 47 (eds. Devine, P. \& Plant, A.) 1-53 (Academic Press, 2013).

110. Hughes, M. E., Waite, L. J., Hawkley, L. C. \& Cacioppo, J. T. A short scale for measuring loneliness in large surveys: results from two population-based studies. Res. Aging 26, 655-672 (2016).

111. Lytle, M. C., Blosnich, J. R., Luca, S. M. D. \& Brownson, C. Association of religiosity with sexual minority suicide ideation and attempt. Am. J. Prev. Med. 54, 644-651 (2018).

112. Ajzen, I. \& Madden, T. J. Prediction of goal-directed behavior: attitudes, intentions, and perceived behavioral control. J. Exp. Soc. Psychol. 22, 453-474 (1986).

113. Webb, T. L. \& Sheeran, P. Does changing behavioral intentions engender behavior change? A meta-analysis of the experimental evidence. Psychol. Bull. 132, 249-268 (2006).

114. Fishbein, M. \& Ajzen, I. Predicting and Changing Behavior: The Reasoned Action Approach (Psychology Press, 2011).

115. Thompson, R. R., Jones, N. M., Holman, E. A. \& Silver, R. C. Media exposure to mass violence events can fuel a cycle of distress. Sci. Adv. 5 , eaav3502 (2019).

116. Dienes, Z. Using Bayes to get the most out of non-significant results. Front. Psychol. 5, 781 (2014).

117. Morey, R. D. \& Rouder, J. N. Baysefactor: computation of Bayes Factors for common designs. R package version 0.9.12-4.2 https://cran.r-project.org/ package $=$ BayesFactor (2018).

118. Dienes, Z. How do I know what my theory predicts? Adv. Methods Pract. Psychol. Sci. 4, 364-377 (2019).

119. Bürkner, P.-C. brms: an R package for Bayesian multilevel models using Stan. J. Stat. Softw. 80, 1-28 (2017).

120. Makowski, D., Ben-Shachar, M. \& Lüdecke, D. bayestestR: describing effects and their uncertainty, existence and significance within the Bayesian framework. J. Open Source Softw. 4, 1541 (2019).

121. Gronau, Q. F., Singmann, H. \& Wagenmakers, E.-J. bridgesampling: an $R$ package for estimating normalizing constants. J. Stat. Softw. 92, 1-29 (2020).

122. Vishkin, A., Hasson, Y., Millgram, Y. \& Tamir, M. One size does not fit all: tailoring cognitive reappraisal to different emotions. Pers. Soc. Psychol. Bull. 46, 469-484 (2019).

123. Anvari, F. \& Lakens, D. Using anchor-based methods to determine the smallest effect size of interest. J. Exp. Soc. Psychol. 96, 104159 (2021).

124. Kvarven, A., Strømland, E. \& Johannesson, M. Comparing meta-analyses and preregistered multiple-laboratory replication projects. Nat. Hum. Behav. 4, 423-434 (2020).

125. Shiota, M. N. \& Levenson, R. W. Turn down the volume or change the channel? Emotional effects of detached versus positive reappraisal. J. Pers. Soc. Psychol. 103, 416-429 (2012).

126. Fetzer, T. et al. Global behaviors and perceptions in the COVID-19 pandemic. Preprint at SSRN https://ssrn.com/abstract=3594262 (2020).

127. Klein, R. A. et al. Many Labs 2: investigating variation in replicability across samples and settings. Adv. Methods Pract. Psychol. Sci. 1, 443-490 (2018).
128. Ellsworth, P. C. \& Scherer, K. R. in Handbook of affective sciences 572-595 (Oxford Univ. Press, 2003).

129. Green, P. \& MacLeod, C. J. SIMR: an R package for power analysis of generalized linear mixed models by simulation. Methods Ecol. Evol. 7, 493-498 (2016)

130. Pordes, R. et al. The open science grid. J. Phys. Conf. Ser. 78, 012057 (2007)

131. Sfligoi, I. et al. The pilot way to grid resources using glideinWMS. In 2009 WRI World Congress on Computer Science and Information Engineering (eds Burgin, M. et al.) 428-432 (IEEE, 2009).

132. Jeffreys, H. Theory of Probability (Oxford Univ. Press, 1961).

133. Brauer, M. \& Curtin, J. J. Linear mixed-effects models and the analysis of nonindependent data: a unified framework to analyze categorical and continuous independent variables that vary within-subjects and/or within-items. Psychol. Methods 23, 389-411 (2018).

\section{Acknowledgements}

This project was supported by funds from: the Amazon Web Services (AWS) Imagine Grant (to E.M.B.); the Japan Society for the Promotion of Science Grants-in-Aid for Scientific Research (JSPS KAKENHI; 16h03079, 17h00875, 18k12015, and 20h04581 to Y.Y.); the research programme Dipartimenti di Eccellenza from the Ministry of Education University and Research (MIUR to N. Cellini and G.M. and the Department of General Psychology of the University of Padua); statutory funds of the University of Wroclaw (to A. Sorokowska); the Charles University Research Programme PROGRES (Q18 to M. Vranka); the Knut and Alice Wallenberg Foundation (2016:0229 to J.K.O.); the Rubicon Grant (019.183sg.007 to K.v.S.) from the Netherlands Organisation for Scientific Research; the Australian Research Council (dp180102384 to R.M.R.); the US National Institutes of Health (NIMH111640 to M.N.-D.), the Huo Family Foundation to N.J.; the NSF Directorate for Social, Behavioral and Economic Sciences, Division of Social and Economic Sciences (1559511 to J.S.L.); the US National Institutes of Health (RO1-CA-224545 to J.S.L.); Eesti Teadusagentuur-Estonian Research Council (PSG525 to A. Uusberg); the J. William Fulbright Program (to F. Azevedo); the HSE Basic Research Program (to D. Dubrov); Dominican University (a Faculty Development Grant to A. Krafnick); and the French National Research Agency Investissements d'avenir supporting PSF (ANR-15-IDEX-02 to H.I.); the Slovak Research and Development Agency (project no. APVV-20-0319 to M. Adamkovič); the programme FUTURE LEADER of Lorraine Université d'Excellence within the French National Research Agency Investissements d'avenir (ANR-15-IDEX-04-LUE to S.M.). Computation for this research was assisted by: the Harvard Business School compute cluster (HBSGrid); and the Open Science Grid. The Open Science Grid is supported by the National Science Foundation award 1148698 and the US Department of Energy's Office of Science, as well as by the compute resources and assistance of the UW-Madison Center For High Throughput Computing (CHTC) in the Department of Computer Sciences. The CHTC is supported by UW-Madison, the Advanced Computing Initiative, the Wisconsin Alumni Research Foundation, the Wisconsin Institutes for Discovery, and the National Science Foundation, and is an active member of the Open Science Grid, which is supported by the National Science Foundation and the U.S. Department of Energy's Office of Science. We thank data science specialist S. Worthington and the research computing environment at the Institute for Quantitative Social Science, Harvard University and V. Ivanchuk for research assistantship. Our semi-representative panels were made possible by an in-kind purchase from the Leibniz Institute for Psychology (protocol https://doi.org/10.23668/psycharchives.3012); a special grant from the Association for Psychological Science and a fee waiver from Prolific. This work was supported by a grant from the American Psychological Society (granted to the PSA). Further financial support was provided by the PSA and a special crowdfunding campaign initiated by the PSA. We thank Amazon Web Services for help with server needs, the Leibniz Institute for Psychology (ZPID) for help with data collection via the organization and implementation of semi-representative panels, Prolific Inc. for offering discounted recruitment, and Harvard University's Institute for Quantitative Social Sciences for statistical consulting. Finally, this research was supported by resources from the Open Science Grid, which is supported by National Science Foundation award 1148698, and the U.S. Department of Energy's Office of Science. Beyond those roles already acknowledged, the funders had no role in study design, data collection and analysis, decision to publish or preparation of the manuscript.

\section{Author contributions}

Conceptualization: K. Wang, A. Goldenberg, C.A.D., A. Uusberg, J.S.L. and J.J.G. Data curation: E.M.B. and P.S.F. Formal analysis: K. Wang, P.S.F. and B. Palfi. Funding acquisition: C.R.C., E.M.B., P.S.F. and H.I. Investigation: J.K.M., L.E., D.H.O., E.A.J., E.O.L.G., J.P.W., K. Desai, E.K., M. Pantazi, N. Pilecka, G.M.M., E.A., M. Adamkovič, M. Roczniewska, C. Reyna, A.P.K., M. Westerlund, L.A., S.P., A.I.A., N.C.A., C.E.O., I.L.G.N., I. Dalgar, P.M.M., F.F., M. Willis, A.C.S., A. Mokady, N.R., M.R.V., N.L.N., M. Parzuchowski, M.F.E.B., M. Vranka, M.B.K., I.R., M. Harutyunyan, E.Y., M. Becker, E. Manunta, G.K., D. Marko, K.E., D.M.G.L., A. Findor, K.P., A.T.L., J.J.B.A., M.S. Ortiz, Z.V., E.P., M. Voracek, C.L., M.G., J.V.V., G.M., N. Cellini, S.-C.C., J.Z., K.M., N.L., A. Karababa, L. Boucher, W.M.C., J. Bavolar, R.M.R., I.D.S., T.J.H., S. Azouaghe, R.M., C.G., C.S.S., G.A., W.J.-L., M. Bradford, L.C.P., J.E.C.V., J.C.V.N., A. Arvanitis, Q.X., R.C., S.Z., Z. Tajchman, I.V., J.M.P., J.R.K., M. Atari, M. Hricova, P.K., J.S., R.-M.R., S.F.M., I. Zakharov, M. A. Koehn, C.E.-S., R.J.C.-J., A.J.K., E.Š., J. Urban, J.R.S., M. Martončik, S.B.O., D. Šakan, A.O.K., J.M.D., I.A.T.A., A. Ferreira, L.B.L., H. Manley, D.Z.R., R.P.M., 
E. Musser, W.C., H.G., S.R.-F., C. Reeck, C. Batres, D.S.A., M.M.B., Z.C., F.V., I. Ziano, M. Tümer, A.C.A.C., D. Dubrov, M.d.C.M.C.T.R., C.A., A. Sacakli, C.D.C., K.L.R., G.S. J.T.P., T.B., H.D., M. Hruška, D. Sousa, K.B., A.N.Z., M.P.-C., M. Bialek, M. Kowal, A. Sorokowska, M. Misiak, D. Mola, M.V.O., P.S.C., A. Belaus, P.A., R.O., L.A.V., P. Szwed, M. Kossowska, J. Kielińska, B. Antazo, G.N., N. Simonovic, J.T., A.G.-K., A.D., K.I., A. Urooj, T.G., A.A., N.A.-A., H.B.K., B.G., T.H., M.V.J., J.B.B., W.J.C., S.Ç., M. Seehuus, A. Khaoudi, A. Bokkour, K.A.E.A., I. Djamai, A. Iyer, N. Parashar, A. Adiguzel, H.E.K., C.B., J.O.N., M.P.-P., A.d.l.R.-G., V.A., N.B., D.G., A. Ivanov, I.P., M. Romanova, I.S., M. Terskova, E.H., A.J., V.S., T.E.S., A.D.A., N.O., N. Say, M. Khosla, A.G.T., F.Y.H.K., G. Bijlstra, F. Mosannenzadeh, B.B.B., U.-D.R., E. Baskin, J.C.-C., B.J.W.D., D. Moreau, C.A.M.S., C.N., H.F., M. Anne, S.M.J.J., N.M.M., Y.K., K.Y., S.D., A.H., M. Vdovic, P.A.G.F., J. Kamburidis, E. Marinova, M.N.-D., N.R.R., A. Stoyanova, K.S., S. Lins, I.R.P., M.K.-T., T.J., J.K.O., O.B., M. Marszalek, S.T., R.A., W.L., J.A., N.V.D., J.A.S., R.S., J. Miranda, K. Damnjanović, S.K.Y., B. Jaeger, D.R., G.P., K. Klevjer, N.S.C.-F., M.F.-A., M.Y.L., A.O.T., M. Toro, L.G.J.D., D.L.G.J.D., S.A.S., R.V., S.M., T.F., A. Bran, D.C.V., L. Vieira, G.L.d.H.C., A. Greenburgh, C.M.W., A.M.T., L. Volz, C. Karaarslan, E.S., T.B.A., M.F.C., T.J.S.L., M.F.F.R., M. Karekla, C. Karashiali, N. Sunami, L.M.J., D. Storage, A. Studzinska, P.H.P.H., D.L.H., M. Sirota, K. Wolfe, F.C., A.T., E.R.A., Y.L., E.C.W., H. Brohmer, G.H., O.D., K.V., G.F., G.A.T., A. Ahmed, M.L., N.T., H. Bai, M. Manavalan, X.S., R.B.W., P.Z., A.D.R., L. Kozma, P.B., G. Banik, M.A.C.V., J. Uttley, B.B., S.N.G., J.K.V., U.S.T., M.C.M., P. Sorokowski, A.G.-B., T.R., J.C., A.A.Ö., J.A.J.-G., M.V.H., T.I., A.L.W., J.P.R., T.O., W.E.D., H.L.U., E.M.B., M.A.P., H.I. and P.S.F. Methodology: K. Wang, A. Goldenberg, C.A.D., A. Uusberg, J.S.L., J.J.G., I.R., F.A., B. Aczel, P. Arriaga, A.G.T., M.A.S., M.C.M., H.L.U., D.M.B.-B. and P.S.F. Project administration: J.K.M., C.Z., S.M.D., M.O., A. Szabelska, G.M.M., A.P.K., I. Dalgar, S.L., N.R., M.R.V., M. Vranka, M. Becker, G.K., E.P., N. Cellini, H. Azab, J.L.B., A.L.T., V.K., M.H.S., E.Š., M. Martončik, D. Dunleavy, K. Kirgizova, F.A., B. Palfi, M.A.M., I.L.P., B. Aczel, P.A., A.G.-K., Y.Y., A. Urooj, L. Bylinina, A.A., B.G., A.D.A., C.A.L., B.I., H.C.-P., J.W.S., J.A., B. Paris, L. Volz, T.B.A., P.H.P.H., F.C., A. Ahmed, L. Kozma, J.E.B., K.K.T., M.A.S., S.M.I., C.R.E., C.R.C., P.R.M., H.L.U., E.M.B., N.A.C., M.A.P., D.M.B.-B., H.I., P.S.F. and H. Moshontz. Resources: B.B.A., M. Bernardo, O.C., K.G., S.M.D., A.P.J., K.R., M. Antoniadi, Z.G., E.K., K.N., O.N.B., M.O., M. Pantazi, N. Pilecka, A. Szabelska, I.M.M.v.S., K.F., A.I.B., G.M.M., M. Adamkovič, M. Roczniewska, A.P.K., M. Westerlund, L.A., S.P., G.A.A., P.D., I. Dalgar, H. Akkas, S.L., I.M.-O., A.C.S., A. Mokady, N.R., M.A. Kurfali, M.R.V., M. Parzuchowski, M. Vranka, I.R., M. Harutyunyan, C. Wang, E.Y., M. Becker, E. Manunta, G.K., D. Marko, A. Findor, A.T.L., J.J.B.A., E.P., R.L., G.M., N. Cellini, S.-C.C., J.Z., H. Azab, A. Karababa, J.L.B., A.L.T., K.v.S., J.V., J. Bavolar, L. Kaliska, V.K., L. Samojlenko, R.P., S.J.G., J. Beitner, L. Warmelink, S. Azouaghe, A. Szala, C.G., C.S.S., O.J.G.-C., J.C.V.N., O.K., J.R.K., C.K.T., C.C.v.B., M.H.S., P.K., J.S., N. Cohen, M.Z., I. Zakharov, E.Š., D. Šakan, J.M.D., D.Z.R., R.P.M., D. Dunleavy, S.R.-F., K. Kirgizova, A. Muminov, F.A., D.S.A., J.M.L., Z.C., M. Tümer, D. Dubrov, M.A.M., B.H., A. Sacakli, W.C.-L., M. Fedotov, M. Wielgus, I.L.P., M. Hruška, B. Aczel, B.S., S. Adamus, K.B., L.M., N.-D.S., A.N.Z., M.P.-C., M. Bialek, M. Kowal, F. Muchembled, R.R.R., P.A., R.O., M. Kossowska, G. C., J. Kielińska, B. Antazo, R.B., S. Stieger, G.N., A.G.-K., A.D., K.I., Y.Y., M. Čadek, J. Messerschmidt, M. Kurfalı, A.A., E. Baklanova, B.G., J.B.B., S.Ç., A. Khaoudi, A. Bokkour, K.A.E.A., I. Djamai, A. Adiguzel, H.E.K., N.B., E.H., V.H.K., V.S., T.E.S., A.D.A., L.M.S.P., D. Krupić, C.A.L., N.J., N. Say, S. Sinkolova, K.J., M. Stojanovska, D. Stojanovska, F. Mosannenzadeh, U.-D.R., B.I., J.C.-C., H.C.-P., M. Topor, Y.K., M. Vdovic, L.A.-B., J. Kamburidis, E. Marinova, M.N.-D., N.R.R., A. Stoyanova, M.K.-T., T.J., O.B., M. Marszalek, W.L., J.A., B.Ž., D. Krupić, K.H., K. Klevjer, D.V., R.V., S.M., A. Bran, L. Vieira, B. Paris, M. Capizzi, G.L.d.H.C., X.D., L. Volz, M.J.B., C. Karaarslan, E.S., T.B.A., M. Korbmacher, J.P.H.V., N. Sunami, S.H., A. Studzinska, P.H.P.H., F.C., O.D., K.V., G.A.T., A. Ahmed, J. Bosch, M. Friedemann, A.D.R., L. Kozma, S.G.A., R.C.C., G. Banik, L.M.R.-B., J.E.B., K.K.T., M.A.S., P.L.G.M., S.M.I., A.G.-B., V.C.A., T.I., L. Suter, M. Bernardo and E.M.B. Supervision: J.K.M., A.L.T., M.H.S., J.W.S., K.K.T., C.R.E., C.R.C., P.R.M., H.L.U., E.M.B., N.A.C., M.A.P., D.M.B.-B., H.I., P.S.F. and H. Moshontz. Visualization: K. Wang, A. Uusberg, A. Goldenberg, C.A.D., J.S.L. and J.J.G. Writing, original draft: K. Wang. Writing, review and editing: K. Wang, A. Goldenberg, C.A.D., A. Uusberg, J.S.L., J.J.G., A. Uusberg, J.K.M., C.Z., B.B.A., M. Bernardo, O.C., L.E., K.G., D.H.O., E.A.J., E.O.L.G., S.M.D., A.P.J., K.R., J.P.W., M. Antoniadi, K. Desai, Z.G., E.K.,
K.N., O.N.B., M.O., M. Pantazi, N. Pilecka, A. Szabelska, I.M.M.v.S., K.F., A.I.B., G.M.M. E.A., M. Adamkovič, M. Roczniewska, C. Reyna, A.P.K., M. Westerlund, L.A., S.P., G.A.A., P.D., A.I.A., N.C.A., C.E.O., I.L.G.N., I. Dalgar, H. Akkas, P.M.M., S.L., I.M.-O., F.F., M. Willis, A.C.S., A. Mokady, N.R., M.A. Kurfali, M.R.V., N.L.N., M. Parzuchowski, M.F.E.B., M. Vranka, M.B.K., I.R., M. Harutyunyan, C. Wang, E.Y., M. Becker, E. Manunta, G.K., D. Marko, K.E., D.M.G.L., A. Findor, K.P., A.T.L., J.J.B.A., M.S. Ortiz, Z.V., E.P., M. Voracek, C.L., M.G., R.L., J.V.V., G.M., N. Cellini, S.-C.C., J.Z., K.M., H. Azab, N.L., A. Karababa, J.L.B., L. Boucher, W.M.C., A.L.T., K.v.S., J.V., J. Bavolar, L. Kaliska, V.K., L. Samojlenko, R.P., S.J.G., J. Beitner, L. Warmelink, R.M.R., I.D.S., T.J.H., S. Azouaghe, R.M., A. Szala, C.G., C.S.S., G.A., W.J.-L., M. Bradford, L.C.P., J.E.C.V., O.J.G.-C., J.C.V.N., O.K., A. Arvanitis, Q.X., R.C., S.Z., Z.T., I.V., J.M.P., J.R.K., C.K.T., C.C.v.B., M. Atari, M.H.S., M. Hricova, P.K., J.S., R.-M.R., N. Cohen, S.F.M., M.Z., I. Zakharov, M.A. Koehn, C.E.-S., R.J.C.-J., A.J.K., E.Š., P.M.I., J. Urban, J.R.S., M. Martončik, S.B.O., D. Šakan, A.O.K., J.M.D., I.A.T.A., A. Ferreira, L.B.L., H. Manley, D.Z.R., R.P.M., E. Musser, D. Dunleavy, W.C., H.G., S.R.-F., C. Reeck, C. Batres, K. Kirgizova, A. Muminov, F.A., D.S.A., M.M.B., J.M.L., Z.C., F.V., I. Ziano, M. Tümer, A.C.A.C., D. Dubrov, M.d.C.M.C.T.R., C.A., B. Palfi, M.A.M., B.H., A. Sacakli, C.D.C., K.L.R., G. S., J.T.P., T.B., W.C.-L., M. Fedotov, H.D., M. Wielgus, I.L.P., M. Hruška, D. Sousa, B. Aczel, B.S., S. Adamus, K.B., L.M., N.-D.S., A.N.Z., M.P.-C., M. Bialek, M. Kowal, A. Sorokowska, M. Misiak, D. Mola, M.V.O., P.S.C., A. Belaus, F. Muchembled, R.R.R., P.A., R.O., L.A.V., P. Szwed, M. Kossowska, G.C., J. Kielińska, B. Antazo, R.B., S. Stieger, G.N., N. Simonovic, J.T., A.G.-K., A.D., K.I., Y.Y., A. Urooj, T.G., M. Čadek, L. Bylinina, J. Messerschmidt, M. Kurfal, A.A., E. Baklanova, N.A.-A., H.B.K., B.G., T.H., M.V.J., J.B.B., W.J.C., S.Ç., M. Seehuus, A. Khaoudi, A. Bokkour, K.A.E.A., I. Djamai, A. Iyer, N. Parashar, A. Adiguzel, H.E.K., C.B., J.O.N., M.P.-P., A.d.l.R.-G., V.A., N.B., D.G., A. Ivanov, I.P., M. Romanova, I.S., M. Terskova, E.H., V.H.K., A.J., V.S., T.E.S., A.D.A., L.M.S.P., D. Krupić, C.A.L., N.J., N.O., N. Say, S. Sinkolova, K.J., M. Stojanovska, D. Stojanovska, M. Khosla, A.G.T., F.Y.H.K., G. Bijlstra, F. Mosannenzadeh, B.B.B., U.-D.R., E. Baskin, B.I., J.C.-C., B.J.W.D., D. Moreau, C.A.M.S., H.C.-P., C.N., H.F., M. Anne, S.M.J.J., M. Topor, N.M.M., Y.K., K.Y., S.D., A.H., M. Vdovic, L.A.-B., P.A.G.F., J. Kamburidis, E. Marinova, M.N.-D., N.R.R., A. Stoyanova, K.S., J.W.S., M.K.-T., T.J., J.K.O., O.B., M. Marszalek, S.T., R.A., W.L., J.A., B.Ž., N.V.D., J.A.S., R.S., J. Miranda, K. Damnjanović, S.K.Y., D. Krupić, K.H., B. Jaeger, D.R., G.P., K. Klevjer, N.S.C.-F., M.F.-A., M.Y.L., A.O.T., M. Toro, L.G.J.D., D.V., S.A.S., R.V., S.M., T.F., A. Bran, D.C.V., L. Vieira, B. Paris, M. Capizzi, G.L.d.H.C., A. Greenburgh, C.M.W., A.M.T., X.D., L. Volz, M.J.B., C. Karaarslan, E.S., T.B.A., M. Korbmacher, M.F.C., T.J.S.L., M.F.F.R., J.P.H.V., M. Karekla, C. Karashiali, N. Sunami, L.M.J., D. Storage, S.H., A. Studzinska, P.H.P.H., D.L.H., M. Sirota, K. Wolfe, F.C., A.T., E.R.A., Y.L., E.C.W., H. Brohmer, G.H., O.D., K.V., G.F., G.A.T., A. Ahmed, M.L., J. Bosch, N.T., H. Bai, M. Manavalan, X.S., R.B.W., P.Z., M. Friedemann, A.D.R., L. Kozma, S.G.A., S. Lins, I.R.P., R.C.C., P.B., G. Banik, L.M.R.-B., M.A.C.V., J. Uttley, J.E.B., K.K.T., B.B., S.N.G., M.A.S., P.L.G.M., J.K.V., U.S.T., S.M.I., M.C.M., P. Sorokowski, A.G.-B., T. Radtke, V.C.A., J.C., A.A.Ö., J.A.J.-G., M.V.H., T.I., A.L.W., J.P.R., T.O., W.E.D., L. Suter, M. Bernardo, C.R.E., C.R.C., P.R.M., H.L.U., E.M.B., N.A.C., M.A.P., D.M.B.-B., H.I., P.S.F. and H. Moshontz.

\section{Competing interests}

The authors declare no competing interests.

\section{Additional information}

Supplementary information The online version contains supplementary material available at https://doi.org/10.1038/s41562-021-01173-x.

Correspondence and requests for materials should be addressed to Jeremy K. Miller.

Peer review information Nature Human Behaviour thanks Elaine Fox, David Mellor, Thomas L. Webb and the other, anonymous, reviewer(s) for their contribution to the peer review of this work. Peer reviewer reports are available.

Reprints and permissions information is available at www.nature.com/reprints. (c) The Author(s), under exclusive licence to Springer Nature Limited 2021, corrected publication 2022 


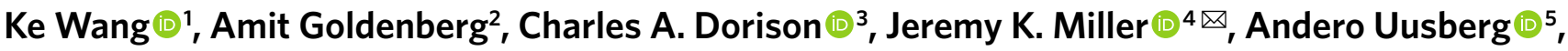
Jennifer S. Lerner ${ }^{6}{ }^{6}$, James J. Gross ${ }^{17}$, Bamikole Bamikole Agesin ${ }^{8}$, Márcia Bernardo9, Olatz Campos ${ }^{10}$, Luis Eudave ${ }^{11}$, Karolina Grzech $\oplus^{12,13}$, Daphna Hausman Ozery' ${ }^{14}$, Emily A. Jackson ${ }^{15}$, Elkin Oswaldo Luis Garcia ${ }^{16}$, Shira Meir Drexler ${ }^{17}{ }^{17}$, Anita Penić Jurković18, Kafeel Rana ${ }^{19}$, John Paul Wilson ${ }^{20}$, Maria Antoniadi ${ }^{21}$, Kermeka Desai ${ }^{22}$, Zoi Gialitaki ${ }^{23}$, Elizaveta Kushnir ${ }^{24}$, Khaoula Nadif ${ }^{25}$, Olalla Niño Bravo ${ }^{26}$, Rafia Nauman ${ }^{27}$, Marlies Oosterlinck ${ }^{28}$, Myrto Pantazi ${ }^{29}$, Natalia Pilecka ${ }^{30}$, Anna Szabelska ${ }^{31}$, I. M. M. van Steenkiste ${ }^{32}$, Katarzyna Filip ${ }^{33}$, Andreea loana Bozdoc ${ }^{34}$, Gabriela Mariana Marcu ${ }^{34,35}$, Elena Agadullina ${ }^{36}$, Matúš Adamkovič ${ }^{37,38}$, Marta Roczniewska $\circledast^{39,40}$, Cecilia Reyna $\oplus^{41}$, Angelos P. Kassianos $\oplus^{42,43}$, Minja Westerlund $\oplus^{44}$, Lina Ahlgren ${ }^{45}$, Sara Pöntinen ${ }^{45}$, Gabriel Agboola Adetula ${ }^{46}$, Pinar Dursun ${ }^{47}$,

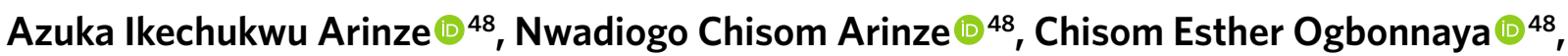
Izuchukwu L. G. Ndukaihe $\oplus^{48}$, Ilker Dalgar $\oplus^{49}$, Handan Akkas $\oplus^{50}$, Paulo Manuel Macapagal ${ }^{51}$, Savannah Lewis $\oplus^{52}$, Irem Metin-Orta $\oplus^{53}$, Francesco Foroni $\oplus^{54}$, Megan Willis $\oplus^{55}$, Anabela Caetano Santos $\oplus^{56,57,58}$, Aviv Mokady $\oplus^{59}$, Niv Reggev ${ }^{60}$, Merve A. Kurfali61, Martin R. Vasilev ${ }^{62}$, Nora L. Nock ${ }^{63}$, Michal Parzuchowski ${ }^{64}$, Mauricio F. Espinoza Barría ${ }^{65}$, Marek Vranka ${ }^{66}$, Markéta Braun Kohlová ${ }^{67}$, Ivan Ropovik ${ }^{68,69}$, Mikayel Harutyunyan ${ }^{70}$, Chunhui Wang ${ }^{71}$, Elvin Yao $\oplus^{72}$, Maja Becker ${ }^{73}$, Efisio Manunta ${ }^{73}$, Gwenael Kaminski ${ }^{73}$, Jordane Boudesseu ${ }^{74}$, Dafne Marko ${ }^{75}$, Kortnee Evans ${ }^{76}$, David M. G. Lewis ${ }^{76,77}$, Andrej Findor ${ }^{78}$, Anais Thibault Landry ${ }^{79}$, John Jamir Benzon Aruta $\oplus^{80}$, Manuel S. Ortiz $\oplus^{81}$, Zahir Vally $\oplus^{82,83 \text {, }}$ Ekaterina Pronizius ${ }^{84}$, Martin Voracek ${ }^{84}$, Claus Lamm ${ }^{84}{ }^{84}$, Maurice Grinberg ${ }^{85}{ }^{85}$, Ranran Li ${ }^{86}$, Jaroslava Varella Valentova $\oplus^{87}$, Giovanna Mioni ${ }^{88}$, Nicola Cellini ${ }^{88,89,90,91}$, Sau-Chin Chen ${ }^{92}$, Janis Zickfeld $\oplus^{93}$, Karis Moon ${ }^{94}$, Habiba Azab ${ }^{95}$, Neil Levy ${ }^{96}$, Alper Karababa ${ }^{97}$, Jennifer L. Beaudry ${ }^{98}{ }^{98}$, Leanne Boucher ${ }^{99}$, W. Matthew Collins ${ }^{100}$, Anna Louise Todsen ${ }^{101}$, Kevin van Schie ${ }^{102,103}$, Jáchym Vintr ${ }^{104}$, Jozef Bavolar ${ }^{105}$, Lada Kaliska ${ }^{106}$, Valerija Križanić ${ }^{107}$, Lara Samojlenko ${ }^{108}$, Razieh Pourafshari ${ }^{109}$, Sandra J. Geiger ${ }^{110}$, Julia Beitner ${ }^{111}$, Lara Warmelink $\oplus^{112}$, Robert M. Ross $\oplus^{113}$, Ian D. Stephen $\left(^{113}\right.$, Thomas J. Hostler ${ }^{114}$, Soufian Azouaghe $\circledast^{115,116}$, Randy McCarthy ${ }^{117}$, Anna Szala $\odot^{118}$, Caterina Grano $\oplus^{119}$, Claudio Singh Solorzano ${ }^{1119}$, Gulnaz Anjum ${ }^{120,121}$, William Jimenez-Leal ${ }^{122}$, Maria Bradford ${ }^{122}$, Laura Calderón Pérez ${ }^{122}$, Julio E. Cruz Vásquez ${ }^{122}$, Oscar J. Galindo-Caballero ${ }^{122}$,

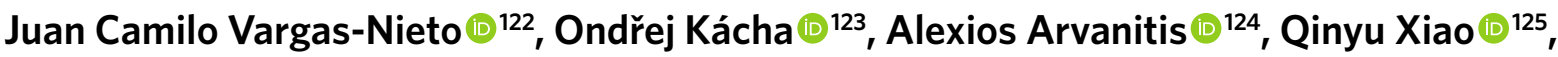
Rodrigo Cárcamo ${ }^{126}$, Saša Zorjan $\left(^{127}\right.$, Zuzanna Tajchman ${ }^{128}$, Iris Vilares ${ }^{128}$, Jeffrey M. Pavlacic $\left({ }^{129}\right.$, Jonas R. Kunst $\circledast^{130}$, Christian K. Tamnes $\left({ }^{130}\right.$, Claudia C. von Bastian ${ }^{131}$, Mohammad Atari ${ }^{132}$, MohammadHasan Sharifian ${ }^{133}$, Monika Hricova ${ }^{105}$, Pavol Kačmár ${ }^{105}$, Jana Schrötter ${ }^{105}$, Rima-Maria Rahal $\oplus^{134}$, Noga Cohen ${ }^{135}$, Saeideh FatahModares ${ }^{136}$, Miha Zrimsek ${ }^{137}$, Ilya Zakharov $\circledast^{138}$, Monica A. Koehn ${ }^{139}$, Celia Esteban-Serna ${ }^{140}$, Robert J. Calin-Jageman $\left({ }^{141}\right.$, Anthony J. Krafnick $\circledast^{141}$, Eva Štrukelj $\circledast^{142}$, Peder Mortvedt Isager ${ }^{143}$, Jan Urban ${ }^{144}$, Jaime R. Silva ${ }^{145,146,147}$, Marcel Martončik (148, Sanja Batić Očovaj ${ }^{149,150}$, Dušana Šakan ${ }^{149,150}$, Anna O. Kuzminska $\oplus^{151}$, Jasna Milosevic Djordjevic ${ }^{152}$, Inês A. T. Almeida ${ }^{153}$, Ana Ferreira $\oplus^{153}$, Ljiljana B. Lazarevic ${ }^{154}$, Harry Manley ${ }^{155}$, Danilo Zambrano Ricaurte ${ }^{156}$, Renan P. Monteiro ${ }^{157}$, Zahra Etabari ${ }^{158}$, Erica Musser $\left({ }^{159}\right.$, Daniel Dunleavy ${ }^{(160}$, Weilun Chou ${ }^{161}$, Hendrik Godbersen $\left({ }^{162}\right.$, Susana Ruiz-Fernández $\oplus^{163,164}$, Crystal Reeck ${ }^{165}$, Carlota Batres ${ }^{166}$, Komila Kirgizova ${ }^{167}$, Abdumalik Muminov ${ }^{168}$, Flavio Azevedo ${ }^{169}$, Daniela Serrato Alvarez ${ }^{170}$, Muhammad Mussaffa Butt $\circledast^{171}$, Jeong Min Lee ${ }^{172}$, Zhang Chen ${ }^{173}$, Frederick Verbruggen ${ }^{173}$, 
Ignazio Ziano ${ }^{174}$, Murat Tümer ${ }^{175}$, Abdelilah C. A. Charyate ${ }^{17}{ }^{176}$, Dmitrii Dubrov ${ }^{36}{ }^{36}$, María del Carmen M. C. Tejada Rivera ${ }^{1077}$, Christopher Aberson ${ }^{10}{ }^{178}$, Bence Pálfi ${ }^{179}$, Mónica Alarcón Maldonado ${ }^{180}$, Barbora Hubena ${ }^{66}$, Asli Sacakli ${ }^{181}$, Chris D. Ceary ${ }^{15}$, Karley L. Richard ${ }^{15}$, Gage Singer ${ }^{182}$, Jennifer T. Perillo ${ }^{183}$, Tonia Ballantyne ${ }^{184}$, Wilson Cyrus-Lai ${ }^{185}$, Maksim Fedotov ${ }^{186}$, Hongfei Du ${ }^{187}$, Magdalena Wielgus ${ }^{188}$, Ilse L. Pit $\mathbb{1}^{189,190}$, Matej Hruška ${ }^{191}$, Daniela Sousa ${ }^{192}$, Balazs Aczel ${ }^{193}$, Nandor Hajdu ${ }^{193}$, Barnabas Szaszi ${ }^{193}$, Sylwia Adamus ${ }^{33}$, Krystian Barzykowski ${ }^{33}$, Leticia Micheli ${ }^{194}$, Nadya-Daniela Schmidt (10) ${ }^{195}$, Andras N. Zsido ${ }^{196}$, Mariola Paruzel-Czachura ${ }^{197}$, Rafał Muda ${ }^{198}$, Michal Bialek ${ }^{199}$, Marta Kowal ${ }^{199}$, Agnieszka Sorokowska ${ }^{199}$, Michal Misiak (199,200, Débora Mola ${ }^{201}$, María Victoria Ortiz ${ }^{201,202}$, Pablo Sebastián Correa $\mathbb{C}^{201}$, Anabel Belaus $\mathbb{B}^{201}$, Fany Muchembled ${ }^{203}$, Rafael R. Ribeiro ${ }^{204}$, Patricia Arriaga ${ }^{204}$, Raquel Oliveira ${ }^{204,205}$, Leigh Ann Vaughn (10) ${ }^{206}$, Paulina Szwed (1) 207 , Małgorzata Kossowska (i] ${ }^{208}$, Gabriela Czarnek (i) 209, Julita Kielińska ${ }^{209}$, Benedict Antazo ${ }^{210}$, Ruben Betlehem ${ }^{211}$, Stefan Stieger ${ }^{212}$, Gustav Nilsonne $\mathbb{B}^{213,214}$, Nicolle Simonovic ${ }^{215}$, Jennifer Taber ${ }^{215}$, Amélie Gourdon-Kanhukamwe ${ }^{216}$, Artur Domurat ${ }^{217}$, Keiko Ihaya ${ }^{218}$, Yuki Yamada ${ }^{219}$, Anum Urooj ${ }^{220}$, Tripat Gill ${ }^{221}$, Martin Čadek ${ }^{222}$, Lisa Bylinina ${ }^{223}$,

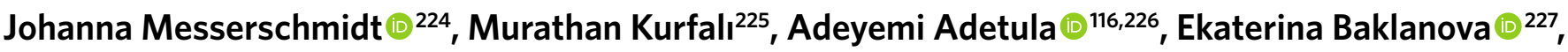
Nihan Albayrak-Aydemir (1] 228, Heather B. Kappes (1) 229, Biljana Gjoneska ${ }^{230}$, Thea House ${ }^{231,232}$, Marc V. Jones ${ }^{114}$, Jana B. Berkessel ${ }^{233}$, William J. Chopik ${ }^{234}$, Sami Çoksan ${ }^{235}$, Martin Seehuus ${ }^{236}$, Ahmed Khaoudi ${ }^{237}$, Ahmed Bokkour ${ }^{237}$, Kanza Ait El Arabi ${ }^{237}$, Ikhlas Djamai ${ }^{237}$,

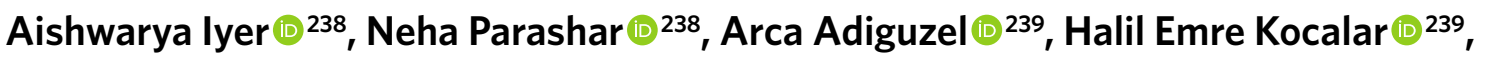
Carsten Bundt $\mathbb{1}^{240,241}$, James O. Norton ${ }^{242}$, Marietta Papadatou-Pastou ${ }^{243}$, Anabel De la Rosa-Gomez ${ }^{244}$, Vladislav Ankushev ${ }^{36}{ }^{36}$, Natalia Bogatyreva ${ }^{36}$, Dmitry Grigoryev ${ }^{36}$, Aleksandr Ivanov ${ }^{36}$, Irina Prusova $\mathbb{1}^{36}$, Marina Romanova $\mathbb{1}^{36}$, Irena Sarieva ${ }^{36}$, Maria Terskova $\mathbb{1}^{36}$, Evgeniya Hristova ${ }^{245}$, Veselina Hristova Kadreva ${ }^{245}$, Allison Janak ${ }^{246}$, Vidar Schei ${ }^{247}$, Therese E. Sverdrup ${ }^{247}$, Adrian Dahl Askelund (10248, Lina Maria Sanabria Pineda (1) 249 , Dajana Krupić ${ }^{250}$, Carmel A. Levitan ${ }^{251}$, Niklas Johannes ${ }^{29}$, Nihal Ouherrou ${ }^{252}$, Nicolas Say ${ }^{253}$, Sladjana Sinkolova ${ }^{254}$, Kristina Janjić ${ }^{254}$, Marija Stojanovska ${ }^{254}$, Dragana Stojanovska ${ }^{254}$, Meetu Khosla ${ }^{255}$, Andrew G. Thomas ${ }^{256}$, Franki Y. H. Kung ${ }^{257}$, Gijsbert Bijlstra ${ }^{258 \text {, }}$ Farnaz Mosannenzadeh (1) 259, Busra Bahar Balci (10) 260,261, Ulf-Dietrich Reips (1) 262, Ernest Baskin (1) 263 , Byurakn Ishkhanyan ${ }^{264,265}$, Johanna Czamanski-Cohen ${ }^{266,267}$, Barnaby James Wyld Dixson ${ }^{268}$, David Moreau ${ }^{269}$, Clare A. M. Sutherland ${ }^{270,271}$, Hu Chuan-Peng ${ }^{272}$, Chris Noone ${ }^{273}$, Heather Flowe $\mathbb{B}^{274}$, Michele Anne $\mathbb{D}^{275}$, Steve M. J. Janssen ${ }^{276}$, Marta Topor $\mathbb{D}^{277}$, Nadyanna M. Majeed ${ }^{278}$, Yoshihiko Kunisato $\mathbb{D}^{279}$, Karen Yu ${ }^{280}$, Shimrit Daches ${ }^{281}$, Andree Hartanto ${ }^{278}$, Milica Vdovic ${ }^{282}$, Lisa Anton-Boicuk ${ }^{283}$, Paul A. G. Forbes ${ }^{283}$, Julia Kamburidis ${ }^{284}$, Evelina Marinova ${ }^{284}$, Mina Nedelcheva-Datsova (1) ${ }^{284}$, Nikolay R. Rachev ${ }^{284}$, Alina Stoyanova ${ }^{284}$, Kathleen Schmidt ${ }^{285}$, Jordan W. Suchow ${ }^{286}$, Maria Koptjevskaja-Tamm (iD) ${ }^{287}$, Teodor Jernsäther ${ }^{214}$, Jonas K. Olofsson ${ }^{20214}$, Olga Bialobrzeska ${ }^{288}$, Magdalena Marszalek $\mathbb{B}^{289}$, Srinivasan Tatachari ${ }^{290}$, Reza Afhami ${ }^{291}$, Wilbert Law ${ }^{292}$, Jan Antfolk ${ }^{293}$, Barbara Žuro ${ }^{294}$, Natalia Van Doren ${ }^{295}$, Jose A. Soto ${ }^{295}$, Rachel Searston ${ }^{296}$, Jacob Miranda ${ }^{297}$, Kaja Damnjanović (1) 298, Siu Kit Yeung (i) 299, Dino Krupić ${ }^{300}$, Karlijn Hoyer ${ }^{301}$, Bastian Jaeger ${ }^{301}$, Dongning Ren ${ }^{302}$, Gerit Pfuhl ${ }^{303}$, Kristoffer Klevjer ${ }^{303}$, Nadia S. Corral-Frías ${ }^{304}$, Martha Frias-Armenta ${ }^{304}$, Marc Y. Lucas ${ }^{305}$, Adriana Olaya Torres ${ }^{177}$, Mónica Toro ${ }^{306}$, Lady Grey Javela Delgado ${ }^{307}$, Diego Vega ${ }^{308}$, Sara Álvarez Solas ${ }^{309}$, Roosevelt Vilar ${ }^{310}$, Sébastien Massoni ${ }^{311}$, Thomas Frizzo ${ }^{311}$, 
Alexandre Bran ${ }^{312}$, David C. Vaidis ${ }^{312}$, Luc Vieira $\oplus^{313}$, Bastien Paris $\oplus^{314}$, Mariagrazia Capizzi $\oplus^{315}$, Gabriel Lins de Holanda Coelho $\oplus^{316}$, Anna Greenburgh $\oplus^{317}$, Cassie M. Whitt ${ }^{318}$, Alexa M. Tullett $\oplus^{319}$, Xinkai Du $\oplus^{320}$, Leonhard Volz $\oplus^{320}$, Minke Jasmijn Bosma ${ }^{321}$, Cemre Karaarslan ${ }^{322}$, Eylül Sarığuz $\circledast^{323}$, Tara Bulut Allred $\circledast^{324}$, Max Korbmacher $\left(^{325}\right.$, Melissa F. Colloff $\left(^{326}\right.$, Tiago J. S. Lima $\oplus^{327}$, Matheus Fernando Felix Ribeiro $\oplus^{328}$, Jeroen P. H. Verharen $\left(^{329}\right.$, Maria Karekla ${ }^{330}$, Christiana Karashiali ${ }^{331}$, Naoyuki Sunami ${ }^{332}$, Lisa M. Jaremka332, Daniel Storage $\mathbb{(}^{333}$, Sumaiya Habib ${ }^{334}$, Anna Studzinska ${ }^{335}$, Paul H. P. Hanel ${ }^{336}$, Dawn Liu Holford $\mathbb{(}^{336}$, Miroslav Sirota $\oplus^{336}$, Kelly Wolfe ${ }^{336}$, Faith Chiu ${ }^{337}$, Andriana Theodoropoulou $\circledast^{338}$, El Rim Ahn ${ }^{339}$, Yijun Lin $\circledast^{340}$, Erin C. Westgate ${ }^{339}$, Hilmar Brohmer $\left(^{341}\right.$, Gabriela Hofer $\left(^{341}\right.$, Olivier Dujols ${ }^{116}$, Kevin Vezirian ${ }^{116}$, Gilad Feldman $\left(^{342}\right.$, Giovanni A. Travaglino $\left(^{343}\right.$, Afroja Ahmed ${ }^{344}$, Manyu Li ${ }^{345}$, Jasmijn Bosch ${ }^{346}$, Nathan Torunsky ${ }^{347}$, Hui Bai $\oplus^{348}$, Mathi Manavalan $\mathbb{1}^{349}$, Xin Song $\oplus^{349}$, Radoslaw B. Walczak $\oplus^{350}$, Przemysław Zdybek $\circledast^{350}$, Maja Friedemann ${ }^{351}$, Anna Dalla Rosa ${ }^{352}$, Luca Kozma ${ }^{196}$, Sara G. Alves ${ }^{353}$, Samuel Lins ${ }^{353}$, Isabel R. Pinto ${ }^{353}$, Rita C. Correia ${ }^{354}$, Peter Babinčák $\circledast^{355}$, Gabriel Banik ${ }^{356}$, Luis Miguel Rojas-Berscia $\oplus^{357,358}$, Marco A. C. Varella ${ }^{87}$, Jim Uttley $\oplus^{359}$, Julie E. Beshears ${ }^{360}$, Katrine Krabbe Thommesen $\left({ }^{101}\right.$, Behzad Behzadnia ${ }^{361}{ }^{361}$, Shawn N. Geniole ${ }^{362}$, Miguel A. Silan ${ }^{363}$, Princess Lovella G. Maturan ${ }^{364}{ }^{364}$, Johannes K. Vilsmeier ${ }^{365}$, Ulrich S. Tran ${ }^{366}$, Sara Morales Izquierdo $\circledast^{367}$, Michael C. Mensink $\oplus^{368}$, Piotr Sorokowski ${ }^{199}$, Agata Groyecka-Bernard $\circledast^{369,370}$, Theda Radtke $\oplus^{371}$, Vera Cubela Adoric $\oplus^{372}$, Joelle Carpentier ${ }^{373}$, Asil Ali Özdoğru $\circledast^{374}$, Jennifer A. Joy-Gaba $\oplus^{375}$, Mattie V. Hedgebeth ${ }^{375}$, Tatsunori Ishii $\oplus^{376}$, Aaron L. Wichman $\left(^{377}\right.$, Jan Philipp Röer ${ }^{378}$, Thomas Ostermann $\left({ }^{378}\right.$, William E. Davis ${ }^{379}$, Lilian Suter ${ }^{380}$, Konstantinos Papachristopoulos ${ }^{381}$, Chelsea Zabel ${ }^{1}$, Sandersan Onie ${ }^{382,383}$, Charles R. Ebersole ${ }^{384}$, Christopher R. Chartier ${ }^{385}$, Peter R. Mallik ${ }^{386}$, Heather L. Urry ${ }^{387}$, Erin M. Buchanan $\oplus^{388}$, Nicholas A. Coles ${ }^{389}$, Maximilian A. Primbs ${ }^{258}$, Dana M. Basnight-Brown $\oplus^{390}$, Hans IJzerman $\oplus^{391}$, Patrick S. Forscher $\oplus^{116}$ and Hannah Moshontz $\oplus^{392}$

\footnotetext{
${ }^{1}$ Harvard Kennedy School, Harvard University, Cambridge, MA, USA. ${ }^{2}$ Harvard Business School, Harvard University, Cambridge, MA, USA. ${ }^{3}$ Kellogg School of Management, Northwestern University, Evanston, IL, USA. ${ }^{4}$ Department of Psychology, Willamette University, Salem, OR, USA. ${ }^{5}$ Institute of Psychology, University of Tartu, Tartu, Estonia. ${ }^{6}$ Harvard Kennedy School and Department of Psychology, Harvard University, Cambridge, MA, USA. ${ }^{7}$ Department of Psychology, Stanford University, Stanford, CA, USA. ${ }^{8}$ Adekunle Ajasin University, Akungba Akoko, Nigeria. ${ }^{9}$ Faculdade de Psicologia e Ciências da Educação, Universidade do Porto, Porto, Portugal. ${ }^{10}$ University of Deusto, Barakaldo, Spain. "University of Navarra, Pamplona, Spain. ${ }^{12}$ University of Valencia, Valencia, Spain. ${ }^{13}$ Stockholm University, Stockholm, Sweden. ${ }^{14}$ California State University, Northridge, Los Angeles, CA, USA. ${ }^{15}$ Indiana University of Pennsylvania, Indiana, PA, USA. ${ }^{16}$ Universidad de Navarra, Pamplona, Spain. ${ }^{17}$ Department of Cognitive Psychology, Institute of Cognitive Neuroscience, Ruhr University Bochum, Bochum, Germany. ${ }^{18}$ Kindergarten Kustošija, Zagreb, Croatia. ${ }^{19} \mathrm{GC}$ University Lahore, Lahore, Pakistan. ${ }^{20}$ Montclair State University, Jersey City, NJ, USA. ${ }^{21}$ European University of Cyprus, Nicosia, Cyprus. ${ }^{22}$ Indiana University of Pennsylvania, Akron, PA, USA. ${ }^{23}$ Leiden University, Culemborg, The Netherlands. ${ }^{24}$ Stockholm, Sweden. ${ }^{25}$ Poggio Imperiale, Italy. ${ }^{26}$ Barakaldo, Spain. ${ }^{27}$ Islamabad, Pakistan. ${ }^{28}$ Gent, Belgium. ${ }^{29} \mathrm{Oxford}$ Internet Institute, University of OxfordOxford, UK. ${ }^{30}$ Warsaw, Poland. ${ }^{31}$ Queen's University Belfast, Belfast, Ireland. ${ }^{32}$ Universiteit LeidenLeiden, The Netherlands. ${ }^{33}$ Institute of Psychology, Jagiellonian University, Krakow, Poland. ${ }^{34}$ Department of Psychology, Lucian Blaga University of Sibiu, Sibiu, Romania. ${ }^{35}$ Carol Davila University of Medicine and Pharmacy, Bucharest, Romania. ${ }^{36}$ National Research University Higher School of Economics, Moscow, Russia. ${ }^{37}$ Institute of Psychology, Faculty of Arts, University of Presov, Presov, Slovakia. ${ }^{38}$ Institute of Social Sciences, CSPS Slovak Academy of Sciences, Košice, Slovakia. ${ }^{39}$ Department of Psychology, SWPS University of Social Sciences and Humanities, Sopot, Poland. ${ }^{40}$ Department of Learning, Informatics, Management and Ethics, Karolinska Institutet, Stockholm, Sweden. ${ }^{41}$ Instituto de Investigaciones Psicológicas (IIPSI), Consejo Nacional de Investigaciones Científicas y Técnicas (CONICET)-UNC, Córdoba, Argentina. ${ }^{42}$ Department of Psychology, University of Cyprus, Nicosia, Cyprus. ${ }^{43}$ Department of Applied Health Research, University College London, London, UK. ${ }^{44}$ Åbo Akademi University, Turku, Finland. ${ }^{45}$ Faculty of Arts, Psychology and Theology, Åbo Akademi University, Turku, Finland. ${ }^{46}$ Department of Pure and Applied Psychology, Faculty of Social and Management Sciences, Adekunle Ajasin University, Akungba, Nigeria. ${ }^{47}$ Department of Psychology, Afyon Kocatepe University, Afyonkarahisar, Turkey. ${ }^{48}$ Alex Ekwueme Federal University Ndufu-Alike, Ndufu-Alike, Nigeria. ${ }^{49}$ Department of Psychology, Ankara Medipol University, Ankara, Turkey. ${ }^{50}$ MIS Department, Ankara Science University, Ankara, Turkey. ${ }^{51}$ School of Psychology, Arellano University, Manila, Philippines. ${ }^{52}$ Ashland University, Ashland, OH, USA. ${ }^{53}$ Department of Psychology, Atilim University, Ankara, Turkey. ${ }^{54}$ Australian Catholic University, North Sydney, New South Wales, Australia. ${ }^{55}$ School of Behavioural and Health Sciences, Australian Catholic University, North Sydney, New South Wales, Australia. ${ }^{56}$ Aventura Social and DESSH, Faculty of Human Kinetics, University of Lisbon,
} 
Lisbon, Portugal. ${ }^{57}$ Institute of Environmental Health, Medicine Faculty, University of Lisbon, Lisbon, Portugal. ${ }^{58}$ ISCTE, Instituto Universitário de Lisboa, Lisbon, Portugal. ${ }^{59}$ Department of Psychology, Ben Gurion University, Beersheba, Israel. ${ }^{60}$ Department of Psychology and Zlotowski Center for Neuroscience, Ben Gurion University, Beersheba, Israel. ${ }^{61}$ Department of Political Science, Bilkent University, Ankara, Turkey. ${ }^{62}$ Department of Psychology, Bournemouth University, Poole, UK. ${ }^{63}$ Case Western Reserve University, Cleveland, OH, USA. ${ }^{64}$ Center for Research on Cognition and Behavior, SWPS University of Social Sciences and Humanities in Sopot, Sopot, Poland. ${ }^{65}$ Centre of Attachment and Emotional Regulation (CARE), Universidad del Desarrollo, Las Condes, Chile. ${ }^{66}$ Charles University, Prague, Czechia. ${ }^{67}$ Environment Center, Charles University, Prague, Czechia. ${ }^{68}$ Institute for Research and Development of Education, Faculty of Education, Charles University, Prague, Czechia. ${ }^{69}$ Faculty of Education, University of Prešov, Prešov, Slovakia. ${ }^{70}$ Institute of Economic Studies, Charles University, Prague, Czechia. ${ }^{71}$ Chinese Center of Disease Prevention and Control, Beijing, China. ${ }^{72} \mathrm{Claremont}$ Graduate University, Claremont, CA, USA. ${ }^{73} \mathrm{CLLE}$, Université de Toulouse, Toulouse, France. ${ }^{74}$ Facultad de Psicología, Instituto de Investigación Científica, Universidad de Lima, Lima, Perú. ${ }^{75}$ Cognitive Science, Faculty of Education, University of Ljubljana, Ljubljana, Slovenia. ${ }^{76}$ College of Science, Health, Engineering and Education, Murdoch University, Perth, Western Australia, Australia. ${ }^{77}$ Centre for Healthy Ageing, Health Futures Institute, Murdoch University, Perth, Western Australia, Australia. ${ }^{78}$ Faculty of Social and Economic Sciences, Comenius University in Bratislava, Bratislava, Slovakia. ${ }^{79}$ Concordia University, Montreal, Quebec, Canada. ${ }^{80}$ De La Salle University, Manila, Philippines. ${ }^{81}$ Departamento de Psicología, Laboratorio de Estrés y Salud, Universidad de La Frontera, Temuco, Chile. ${ }^{82}$ Department of Clinical Psychology, United Arab Emirates University, Al Ain, UAE. ${ }^{83}$ Wolfson College, University of Oxford, Oxford, UK. ${ }^{84}$ Department of Cognition, Emotion and Methods in Psychology, Faculty of Psychology, University of Vienna, Vienna, Austria. ${ }^{85}$ Department of Cognitive Science and Psychology, Research Center for Cognitive Science, New Bulgarian University, Sofia, Bulgaria. ${ }^{86}$ Department of Experimental and Applied Psychology, Vrije Universiteit Amsterdam, Amsterdam, The Netherlands. ${ }^{87}$ Department of Experimental Psychology, Institute of Psychology, University of São Paulo, São Paulo, Brazil. ${ }^{88}$ Department of General Psychology, University of Padua, Padua, Italy. ${ }^{89}$ Department of Biomedical Sciences, University of Padua, Padua, Italy. ${ }^{90}$ Padova Neuroscience Center, University of Padua, Padua, Italy. ${ }^{91}$ Human Inspired Technology Center, University of Padua, Padua, Italy. ${ }^{92}$ Department of Human Development and Psychology, Tzu-Chi University, Hualien, Taiwan. ${ }^{93}$ Department of Management, Aarhus University, Aarhus, Denmark. ${ }^{94}$ Department of Management, Kingston University London, Kingston, UK. ${ }^{95}$ Department of Neurosurgery, Baylor College of Medicine, Houston, TX, USA. ${ }^{96}$ Department of Philosophy, Macquarie University, Sydney, New South Wales, Australia. ${ }^{97}$ Department of Psychological Counselling and Guidance, Faculty of Education, Muğla Sıtkı Koçman University, Muğla, Turkey. ${ }^{98}$ Department of Psychological Sciences, Swinburne University of Technology, Melbourne, Victoria, Australia. ${ }^{99}$ Department of Psychology and Neuroscience, Nova Southeastern University, Pembroke Pines, FL, USA. ${ }^{100}$ Department of Psychology and Neuroscience, Nova Southeastern University, Fort Lauderdale, FL, USA. ${ }^{101}$ School of Psychology and Neuroscience, University of St Andrews, St Andrews, UK. ${ }^{102}$ Department of Psychology, Education and Child Studies, Erasmus School of Social and Behavioural Sciences, Erasmus University, Rotterdam, The Netherlands. ${ }^{103}$ MRC Cognition and Brain Sciences Unit, University of Cambridge, Cambridge, UK. ${ }^{104}$ Department of Psychology, Faculty of Arts, Charles University, Prague, Czechia. ${ }^{105}$ Department of Psychology, Faculty of Arts, Pavol Jozef Šafárik University in Košice, Košice, Slovakia. ${ }^{106}$ Department of Psychology, Faculty of Education, Matej Bel University, Banská Bystrica, Slovakia. ${ }^{107}$ Department of Psychology, Faculty of Humanities and Social Sciences, J. J. Strossmayer University of Osijek, Osijek, Croatia. ${ }^{108}$ Department of Psychology, Faculty of Mathematics, Natural Sciences and Information Technologies, University of Primorska, Koper, Slovenia. ${ }^{109}$ Department of Psychology, Faculty of Psychology and Education, University of Tehran, Tehran, Iran. ${ }^{10}$ Department of Psychology, Faculty of Social and Behavioural Sciences, University of Amsterdam, Amsterdam, The Netherlands. "'Department of Psychology, Goethe University Frankfurt, Frankfurt, Germany. ${ }^{12}$ Department of Psychology, Lancaster University, Lancaster, UK. ${ }^{113}$ Department of Psychology, Macquarie University, Sydney, New South Wales, Australia. ${ }^{114}$ Department of Psychology, Manchester Metropolitan University, Manchester, UK. ${ }^{115}$ Department of Psychology, Mohammed V University in Rabat, Rabat, Morocco. ${ }^{116} \mathrm{LIP} / \mathrm{PC} 2 \mathrm{~S}$, Université Grenoble Alpes, Grenoble, France. ${ }^{177}$ Department of Psychology, Northern Illinois University, DeKalb, IL, USA. ${ }^{118}$ Department of Psychology, Oakland University, Oakland County, MI, USA. ${ }^{119}$ Department of Psychology, Sapienza University, Rome, Italy. ${ }^{120}$ Department of Psychology, Simon Fraser University, Burnaby, British Columbia, Canada. ${ }^{121}$ Department of Economics \& Social Sciences, Institute of Business Administration, Karachi, Pakistan. ${ }^{122}$ Department of Psychology, Universidad de los Andes, Bogotá, Colombia. ${ }^{123}$ Department of Psychology, University of Cambridge, Cambridge, UK. ${ }^{124}$ Department of Psychology, University of Crete, Rethymno, Greece. ${ }^{125}$ Department of Psychology, University of Hong Kong, Hong Kong, Hong Kong. ${ }^{126}$ Department of Psychology, University of Magallanes, Punta Arenas, Chile. ${ }^{127}$ Department of Psychology, University of Maribor, Maribor, Slovenia. ${ }^{128}$ Department of Psychology, University of Minnesota, Minneapolis, MN, USA. ${ }^{129}$ Department of Psychology, University of Mississippi, Oxford, MS, USA. ${ }^{130}$ Department of Psychology, University of Oslo, Oslo, Norway. ${ }^{131}$ Department of Psychology, University of Sheffield, Sheffield, UK. ${ }^{132}$ Department of Psychology, University of Southern California, Los Angeles, CA, USA. ${ }^{133}$ Department of Psychology, University of Tehran, Tehran, Iran. ${ }^{134}$ Department of Social Psychology, Tilburg University, Bonn, Germany. ${ }^{135}$ Department of Special Education and The Edmond J. Safra Brain Research Center for the Study of Learning Disabilities, University of Haifa, Tel Aviv, Israel. ${ }^{136}$ Department of Sport Management, Faculty of Physical Education and Sport Science, Urmia University, Urmia, Iran. ${ }^{137}$ Department of Translation Studies, Faculty of Arts, University of Ljubljana, Ljubljana, Slovenia. ${ }^{138}$ Developmental Behavioral Genetics Laboratory, Psychological Institute of the Russian Academy of Education, Moscow, Russia. ${ }^{139}$ Discipline of Psychology, Faculty of Health, University of Canberra, Canberra, Australia. ${ }^{140}$ Division of Psychology and Language Sciences, University College London, London, UK. ${ }^{141}$ Psychology Department, Dominican University, River Forest, IL, USA. ${ }^{142}$ Dynamic and Clinical Psychology, Sapienza University of Rome, Rome, Italy. ${ }^{143}$ Department of Industrial Engineering and Innovation Sciences, Eindhoven University of Technology, Eindhoven, The Netherlands.

${ }^{144}$ Environment Center, Charles University, Prague, Czechia. ${ }^{145}$ Facultad de Psicología, Universidad del Desarrollo, Concepción, Chile. ${ }^{166} \mathrm{Clínica}$ Alemana de Santiago, Santiago, Chile. ${ }^{147}$ Sociedad Chilena de Desarrollo Emocional, Santiago, Chile. ${ }^{148}$ Faculty of Arts, University of Presov, Prešov, Slovakia. ${ }^{149}$ Dr Lazar Vrkatic Faculty of Legal and Business Studies, Novi Sad, Serbia. ${ }^{150}$ Department of Psychology, Serbia Union University, Novi Sad, Serbia. ${ }^{151}$ Faculty of Management, University of Warsaw, Warsaw, Poland. ${ }^{152}$ Faculty of Media and Communication, Singidunum University, Belgrade, Serbia. ${ }^{153}$ Faculty of Medicine FMUC, Institute of Nuclear Sciences Applied to Health ICNAS, Coimbra Institute for Biomedical Imaging and Translational Research CIBIT, University of Coimbra, Coimbra, Portugal. ${ }^{154}$ Faculty of Philosophy, University of Belgrade, Belgrade, Serbia. ${ }^{155}$ Faculty of Psychology, Chulalongkorn University, Bangkok, Thailand. ${ }^{156}$ Faculty of Psychology, Fundación Universitaria Konrad Lorenz, Bogotá, Colombia. ${ }^{157}$ Department of Psychology, Federal University of Mato Grosso, Cuiabá, Brazil. ${ }^{158}$ Ferdowsi University of Mashhad, Mashhad, Iran. ${ }^{159}$ Center for Children and Families, Department of Psychology, Florida International University, Miami, FL, USA. ${ }^{160}$ Center for Translational Behavioral Science, Florida State University, Tallahassee, FL, USA. ${ }^{161}$ Department of Psychology, Fo Guang University, Yilan County, Taiwan. ${ }^{162} \mathrm{FOM}$ University of Applied Sciences, Essen, Germany. ${ }^{163} \mathrm{FOM}$ University of Applied Sciences, Essen; Leibniz-Institut für Wissensmedien, Tübingen, Germany. ${ }^{164}$ LEAD Research Network, Eberhard Karls University, Tübingen, Germany. ${ }^{165} \mathrm{Fox}$ School of Business, Temple University, Philadelphia, PA, USA. ${ }^{166}$ Department of Psychology, Franklin and Marshall College, Lancaster, PA, USA. ${ }^{167}$ Florence, Italy. ${ }^{168}$ Samarkand, Uzbekistan. ${ }^{169}$ Friedrich Schiller University Jena, Jena, Germany. ${ }^{170}$ Fundación Universitaria Konrad Lorenz, Bogotá, Colombia. ${ }^{171} \mathrm{GC}$ University, Lahore, Pakistan. ${ }^{172}$ Department of Psychology, Georgia State University, Atlanta, GA, USA. ${ }^{173}$ Department of Experimental Psychology, Ghent University, Gent, Belgium. ${ }^{174}$ Grenoble Ecole de Management, Grenoble, France. ${ }^{175}$ Department of Anesthesiology and Reanimation, Hacettepe University, Ankara, Turkey. ${ }^{176} \mathrm{Higher}$ College of Education and Training, Ibn Tofail University, Kenitra, Morocco. ${ }^{177}$ Universidad del Desarrollo, Santiago, Chile. ${ }^{178} \mathrm{Humboldt}$ State University, Arcata, CA, USA. ${ }^{179}$ Department of Surgery and Cancer, Imperial College London, London, UK. ${ }^{180}$ Puebla, Mexico. ${ }^{181}$ Ankara, Turkey. ${ }^{182}$ Department of Psychology, Indiana University of Pennsylvania, Saint Michael, PA, USA. ${ }^{183}$ Department of Psychology, Indiana 
University of Pennsylvania, Indiana, PA, USA. ${ }^{184}$ Indiana University Pennsylvania, Indiana, PA, USA. ${ }^{185}$ INSEAD, Singapore, Singapore. ${ }^{186}$ Institute for Linguistic Studies, Russian Academy of Sciences, Saint Petersburg, Russia. ${ }^{187}$ Institute of Advanced Studies in Humanities and Social Sciences, Beijing Normal University at Zhuhai, Guangzhou, China. ${ }^{188}$ Institute of Applied Psychology, Jagiellonian University, Kraków, Poland. ${ }^{189}$ Institute of Cognitive and Evolutionary Anthropology, University of Oxford, Oxford, UK. ${ }^{190} \mathrm{Calleva}$ Research Centre for Evolution and Human Sciences, Magdalen College, University of Oxford, Oxford, UK. ${ }^{191}$ Institute of European Studies and International Relations, Faculty of Social and Economic Sciences, Comenius University in Bratislava, Bratislava, Slovakia. ${ }^{192}$ Institute of Nuclear Sciences Applied to Health ICNAS, Coimbra Institute for Biomedical Imaging and Translational Research CIBIT, University of Coimbra, Coimbra, Portugal. ${ }^{193}$ Institute of Psychology, ELTE Eötvös Loránd University, Budapest, Hungary. ${ }^{194}$ Institute of Psychology, Leibniz University Hannover, Hannover, Germany. ${ }^{195}$ Institute of Psychology, University of Hildesheim, Hildesheim, Germany. ${ }^{196}$ Institute of Psychology, University of Pécs, Pécs, Hungary. ${ }^{197}$ Institute of Psychology, University of Silesia in Katowice, Katowice, Poland. ${ }^{198}$ Faculty of Economics, Maria Curie-Sklodowska University, Lublin, Poland. ${ }^{199}$ Institute of Psychology, University of Wroclaw, Wrocław, Poland. ${ }^{200}$ School of Anthropology and Museum Ethnography, University of Oxford, Oxford, UK. ${ }^{201}$ Instituto de Investigaciones Psicológicas (IIPsi), Universidad Nacional de Córdoba-Conicet, Córdoba, Argentina. ${ }^{202}$ Facultad de Psicología, Universidad Nacional de Córdoba, Córdoba, Argentina. ${ }^{203}$ Instituto Tecnológico de Estudios Superiores de Monterrey, Hermosillo, Mexico. ${ }^{204} \mathrm{CIS}$-IUL, Iscte-Instituto Universitário de Lisboa, Lisbon, Portugal. ${ }^{205}$ Intelligent Agents and Synthetic Characters Group (GAIPS), INESC-ID, Lisbon, Portugal. ${ }^{206}$ |thaca College, Ithaca, NY, USA. ${ }^{207}$ Jagiellonian University, Kraków, Poland. ${ }^{208}$ Department of Philosophy, Institute of Psychology, Jagiellonian University in Krakow, Kraków, Poland. ${ }^{209}$ Instytute of Psychology, Jagiellonian University, Czestochowa, Poland. ${ }^{210}$ Department of Psychology, Jose Rizal University, Pasig City, Philippines. ${ }^{211}$ Department of Psychology, Faculty of Humanities and Social Sciences, Josip Juraj Strossmayer University of Osijek, Osijek, Croatia. ${ }^{212}$ Department of Psychology and Psychodynamics, Karl Landsteiner University of Health Sciences, Krems an der Donau, Austria. ${ }^{213}$ Department of Clinical Neuroscience, Karolinska Institutet, Solna, Sweden. ${ }^{214}$ Department of Psychology, Stockholm University, Stockholm, Sweden. ${ }^{215}$ Department of Psychological Sciences, Kent State University, Kent, OH, USA. ${ }^{216}$ Institute for Globally Distributed Open Research and Education (IGDORE), Kingston University, London, UK. ${ }^{217}$ Centre for Economic Psychology and Decision Sciences, Kozminski University, Warsaw, Poland. ${ }^{218} \mathrm{Admission}$ Center, Kyushu University, Fukuoka, Japan. ${ }^{219} \mathrm{Faculty}$ of Arts and Science, Kyushu University, Fukuoka, Japan. ${ }^{220}$ La Trobe University, Melbourne, Victoria, Australia. ${ }^{221}$ Lazaridis School of Business and Economics, Wilfrid Laurier University, Waterloo, Ontario, Canada. ${ }^{222}$ Carnegie School of Sport, Leeds Beckett University, London, UK. ${ }^{223}$ Leiden University, Utrecht, The Netherlands. ${ }^{224}$ Institute of Psychology, Leipzig University, Heidelberg, Germany. ${ }^{225}$ Linguistics Department, Stockholm University, Stockholm, Sweden. ${ }^{226}$ Department of Psychology, Alex Ekwueme Federal University, Abakaliki, Nigeria. ${ }^{227}$ Institute of Asian and African Studies, Lomonosov Moscow State University, Moscow, Russia. ${ }^{228}$ London School of Economics and Political Science, London, UK. ${ }^{229}$ Department of Management, London School of Economics and Political Science, London, UK. ${ }^{230}$ Macedonian Academy of Sciences and Arts, Skopje, North Macedonia. ${ }^{231}$ Macquarie University, Sydney, New South Wales, Australia. ${ }^{232}$ University of Bristol, Bristol, UK. ${ }^{233}$ Mannheim Centre for European Social Research, University of Mannheim, Mannheim, Germany. ${ }^{234}$ Department of Psychology, Michigan State University, East Lansing, MI, USA. ${ }^{235}$ Department of Psychology, Middle East Technical University, Ankara, Turkey. ${ }^{236}$ Department of Psychology, Middlebury College; Vermont Psychological Services, University of Vermont, Middlebury, VT, USA. ${ }^{237}$ Mohammed V University in Rabat, Rabat, Morocco. ${ }^{238}$ Sampurna Montfort College, Bangalore, India. ${ }^{239}$ Department of Psychological Counseling and Guidance, Muğla Sıtkı Koçman University, Muğla, Turkey. ${ }^{240}$ Multimodal Imaging and Cognitive Control Lab, Department of Psychology, University of Oslo, Oslo, Norway. ${ }^{241}$ Cognitive and Translational Neuroscience Cluster, Department of Psychology, University of Oslo, Oslo, Norway. ${ }^{242}$ College of Science, Health, Engineering and Education, Murdoch University, Lesmurdie, Australia. ${ }^{243}$ National and Kapodistrian University of Athens, Athens, Greece. ${ }^{244} \mathrm{Faculty}$ of Higher Studies "Iztacala", National Autonomous University of Mexico, Mexico City, Mexico. ${ }^{245}$ Department of Cognitive Science and Psychology, New Bulgarian University, Sofia, Bulgaria. ${ }^{246}$ Department of Applied Psychology, New York University, New York, NY, USA. ${ }^{247} \mathrm{NHH}$ Norwegian School of Economics, Department of Strategy and Management, Bergen, Norway. ${ }^{248}$ Nic Waals Institute, Lovisenberg Diaconal Hospital, Oslo, Norway. ${ }^{249}$ Universidad de los Andes, Bogotá, Colombia. ${ }^{250}$ Norvel一Psychological Centre for Counselling and Research, Osijek, Croatia. ${ }^{251}$ Department of Cognitive Science, Occidental College, Los Angeles, CA, USA. ${ }^{252}$ Paul Valery Montpellier University, Montpellier, France. ${ }^{253}$ Prague University of Economics and Business, Prague, Czechia. ${ }^{254}$ PSA Psihesko, Skopje, North Macedonia. ${ }^{255}$ Psychology Department, DRC, University of Delhi, Delhi, India. ${ }^{256}$ Psychology Department, Swansea University, Swansea, UK. ${ }^{257}$ Purdue University, West Lafayette, IN, USA. ${ }^{258}$ Behavioural Science Institute, Radboud University, Nijmegen, The Netherlands. ${ }^{259}$ Behavioural Science Institute, Faculty of Social Sciences, Radboud University, Nijmegen, The Netherlands. ${ }^{260}$ Department of Psychology, Samsun University, Samsun, Turkey. ${ }^{261}$ Department of Psychology, Dokuz Eylül University, Samsun, Turkey. ${ }^{262}$ Research Methods, Assessment, and iScience, Department of Psychology, University of Konstanz, Kreuzlingen, Switzerland. ${ }^{263}$ Saint Joseph's University, Philadelphia, PA, USA. ${ }^{264}$ School of Communication and Culture, Aarhus University, Aarhus, Denmark. ${ }^{265}$ Department of Nordic Studies and Linguistics, University of Copenhagen, Aarhus, Denmark. ${ }^{266}$ School of Creative Arts Therapies, University of Haifa, Haifa, Israel. ${ }^{267}$ Emili Sagol Creative Arts Therapies Research Center, University of Haifa, Haifa, Israel. ${ }^{268}$ School of Health and Behavioural Sciences, University of the Sunshine Coast, Brisbane, Queensland, Australia. ${ }^{269}$ School of Psychology and Centre for Brain Research, The University of Auckland, Auckland, New Zealand. ${ }^{270}$ School of Psychology, King's College, University of Aberdeen, Aberdeen, UK. ${ }^{271}$ School of Psychological Science, University of Western Australia, Perth, Western Australia, Australia. ${ }^{272}$ School of Psychology, Nanjing Normal University, Nanjing, China. ${ }^{273}$ School of Psychology, National University of Ireland, Galway, Galway, Ireland. ${ }^{274}$ School of Psychology, University of Birmingham, Birmingham, AL, USA. ${ }^{275}$ School of Psychology, University of Nottingham Malaysia, Semenyih, Malaysia. ${ }^{276}$ School of Psychology, University of Nottingham Malaysia, Kuala Lumpur, Malaysia. ${ }^{277}$ School of Psychology, University of Surrey, Guildford, UK. ${ }^{278}$ School of Social Sciences, Singapore Management University, Singapore, Singapore. ${ }^{279}$ Department of Psychology, Senshu University, Tokyo, Japan. ${ }^{280}$ Sewanee: The University of the South, Sewanee, TN, USA. ${ }^{281}$ Department of Psychology, Bar-Ilan University, Ramat Gan, Israel. ${ }^{282}$ Department of Psychology, Faculty of Media and Communications, Singidunum University, Belgrade, Serbia. ${ }^{283}$ Social, Cognitive and Affective Neuroscience Unit, Department of Cognition, Emotion, and Methods in Psychology, Faculty of Psychology, University of Vienna, Vienna, Austria. ${ }^{284}$ Department of General, Experimental, Developmental, and Health Psychology, Sofia University St Kliment Ohridski, Sofia, Bulgaria. ${ }^{285}$ School of Psychological and Behavioral Sciences, Southern Illinois University, Carbondale, IL, USA. ${ }^{286}$ School of Business, Stevens Institute of Technology, Hoboken, NJ, USA. ${ }^{287}$ Department of Linguistics, Stockholm University, Stockholm, Sweden. ${ }^{288}$ SWPS University of Social Sciences and Humanities, Warsaw, Poland. ${ }^{289}$ SWPS University of Social Sciences and Humanities, Gdansk, Poland. ${ }^{290}$ T A Pai Management Institute, Manipal, India. ${ }^{291}$ Department of Art Studies, Tarbiat Modares University, Tehran, Iran. ${ }^{292}$ Department of Psychology, The Education University of Hong Kong, Hong Kong, Hong Kong. ${ }^{293}$ The Faculty of Arts, Psychology and Theology, Åbo Akademi University, Turku, Finland. ${ }^{294}$ The Institute of Psychology; Faculty of Humanities and Social Sciences, University of Osijek, Osijek, Croatia. ${ }^{295}$ Department of Psychology, The Pennsylvania State University, State College, PA, USA. ${ }^{296}$ The University of Adelaide, Adelaide, South Australia, Australia. ${ }^{297}$ Department of Psychology, The University of Alabama, Tuscaloosa, AL, USA. ${ }^{298}$ Laboratory for Experimental Psychology, Institute of Philosophy, Department of Psychology, Faculty of Philosophy, The University of Belgrade, Beograd-Stari Grad, Serbia. ${ }^{299}$ The University of Hong Kong, Hong Kong, Hong Kong. ${ }^{300}$ Faculty of Humanities and Social Science, The University of Osijek, Osijek, Croatia. ${ }^{301}$ Tilburg University, Tilburg, The Netherlands. ${ }^{302}$ Department of Social Psychology, Tilburg University, Tilburg, The Netherlands. ${ }^{303}$ Department of Psychology, UiT The Arctic University of Norway, Troms $ø$, Norway. ${ }^{304}$ Universidad de Sonora, Hermosillo, Mexico. ${ }^{305}$ Department of Psychology, Universidad de Sonora, Hermosillo, Mexico. ${ }^{306}$ Centro de Apego y Regulación Emocional, Facultad de Psicología, Universidad Del Desarrollo, Santiago, Chile. ${ }^{307}$ Programa de Psicología, Universidad del Rosario, Bogotá, Colombia. ${ }^{308}$ Universidad Latina de Costa Rica, San Jose, Costa Rica. ${ }^{309}$ Grupo de investigación en Biogeografía 
y Ecología Espacial (BioGeoE2), Universidad Regional Amazónica Ikiam, Tena, Ecuador. ${ }^{310}$ Universidade Cruzeiro do Sul, São Paulo, Brazil. ${ }^{311}$ Université de Lorraine; CNRS, BETA, Université de Strasbourg, Nancy, France. ${ }^{312}$ Université de Paris, Paris, France. ${ }^{313}$ Université de Paris, Strasbourg, France. ${ }^{314}$ Université Grenoble Alpes, Grenoble, France. ${ }^{315}$ Université Paul Valéry Montpellier, Granada, Spain. ${ }^{316}$ University College Cork, Cork, Ireland. ${ }^{317}$ Department of Experimental Psychology, University College London, London, UK. ${ }^{318}$ University of Alabama, Tuscaloosa, AL, USA. ${ }^{319}$ Department of Psychology, University of Alabama, Tuscaloosa, AL, USA. ${ }^{320}$ University of Amsterdam, Amsterdam, The Netherlands. ${ }^{321}$ Department of Psychology, University of Amsterdam, Amsterdam, The Netherlands. ${ }^{322}$ Department of Psychology, Institute of Social Sciences, University of Bașkent, Ankara, Turkey. ${ }^{323}$ Department of Psychology, Institute of Social Sciences, University of Bașkent, C,ankaya, Turkey. ${ }^{324}$ Laboratory for Research of Individual Differences, Faculty of Philosophy, University of Belgrade, Belgrade, Serbia. ${ }^{325}$ Department of Biological and Medical Psychology, Faculty of Psychology, University of Bergen, Bergen, Norway. ${ }^{326}$ University of Birmingham, Birmingham, UK. ${ }^{327}$ Department of Social and Work Psychology, University of Brasília, Brasília, Brazil. ${ }^{328}$ Institute of Psychology, University of Brasilia, Uberaba, Brazil. ${ }^{329}$ Department of Molecular and Cell Biology, University of California Berkeley, Berkeley, CA, USA. ${ }^{330}$ University of Cyprus, Nicosia, Cyprus. ${ }^{331}$ Department of Psychology, University of Cyprus, Nicosia, Cyprus. ${ }^{332}$ Department of Psychological and Brain Sciences, University of Delaware, Newark, DE, USA. ${ }^{33}$ Department of Psychology, University of Denver, Denver, CO, USA. ${ }^{334}$ Department of Clinical Psychology, University of Dhaka, Dhaka, Bangladesh. ${ }^{335}$ University of Economics and Human Sciences in Warsaw, Warsaw, Poland. ${ }^{336}$ University of Essex, Colchester, UK. ${ }^{337}$ Department of Language and Linguistics, University of Essex, Colchester, UK. ${ }^{338}$ Department of Psychology, University of Essex, Colchester, UK. ${ }^{339}$ Department of Psychology, University of Florida, Gainesville, USA. ${ }^{340}$ Department of Psychology, University of Florida, New York, NY, USA. ${ }^{34 I}$ Institute of Psychology, University of Graz, Graz, Austria. ${ }^{342}$ University of Hong Kong, Hong Kong, Hong Kong. ${ }^{343}$ School of Psychology, Keynes College, University of Kent, Canterbury, UK. ${ }^{344}$ Department of Psychology, Global MINDS, University of Limerick, Dhaka, Bangladesh. ${ }^{345}$ University of Louisiana at Lafayette, Lafayette, LA, USA. ${ }^{346}$ University of Milan-Bicocca, Milan, Italy. ${ }^{347}$ Department of Psychology, University of Minnesota, Twin Cities, Minneapolis, MN, USA. ${ }^{348}$ University of Minnesota, Twin Cities, Saint Paul, MN, USA. ${ }^{349}$ Department of Psychology, University of Minnesota, Twin Cities, Minneapolis, MN, USA. ${ }^{350}$ Institute of Psychology, University of Opole, Opole, Poland. ${ }^{351}$ University of Oxford, Oxford, UK. ${ }^{352}$ Department of Philosophy, Sociology, Education and Applied Psychology, University of Padova, Zovencedo, Italy. ${ }^{353}$ Center for Psychology, University of Porto, Porto, Portugal. ${ }^{354}$ Center for Psychology, University of Porto, Amarante, Portugal. ${ }^{355}$ Institute of Psychology, Faculty of Arts, University of Presov, Prešov, Slovakia. ${ }^{356}$ Institute of Psychology, University of Presov, Prešov, Slovakia. ${ }^{357}$ School of Languages and Cultures, University of Queensland, Lucia, Queensland, Australia. ${ }^{358}$ Centro de Estudios Orientales, Pontificia Universidad Católica del Perú, Lima, Peru. ${ }^{359}$ School of Architecture, University of Sheffield, Sheffield, UK. ${ }^{360}$ University of Southern Indiana, Greenwood, IN, USA. ${ }^{36}$ University of Tabriz, Tabriz, Iran. ${ }^{362}$ Department of Psychology, University of the Fraser Valley, Surrey, British Columbia, Canada. ${ }^{36}$ University of the Philippines Diliman, Quezon City, Philippines. ${ }^{364}$ Department of Psychology, University of the Philippines Diliman, Metro Manila, Philippines. ${ }^{365}$ Department of Cognition, Emotion, and Methods in Psychology, University of Vienna, Vienna, Austria. ${ }^{366}$ Department of Cognition, Emotion, and Methods in Psychology, School of Psychology, University of Vienna, Vienna, Austria. ${ }^{367}$ University of Warwick, Coventry, UK. ${ }^{368}$ Department of Psychology, University of Wisconsin-Stout, White Bear Township, WI, USA. ${ }^{369}$ Institute of Psychology, University of Wroclaw; Social and Legal Psychology, Johannes Gutenberg University, Wrocław, Poland. ${ }^{370}$ Social and Legal Psychology, Johannes Gutenberg University, Mainz, Germany. ${ }^{371}$ Department of Psychology, University of Wuppertal, Witten, Germany. ${ }^{372}$ Department of Psychology, University of Zadar, Zadar, Croatia. ${ }^{373}$ Department of Organization and Human Resources, School of Management, Université du Québec à Montréal, Montreal, Quebec, Canada. ${ }^{374}$ Department of Psychology, Üsküdar University, Istanbul, Turkey. ${ }^{375}$ Virginia Commonwealth University, Richmond, VA, USA. ${ }^{376}$ Faculty of Science and Engineering, Waseda University, Tokyo, Japan. ${ }^{377}$ Western Kentucky University, Bowling Green, KY, USA. ${ }^{378}$ Department of Psychology and Psychotherapy, Witten/Herdecke University, Witten, Germany. ${ }^{379}$ Department of Psychology, Wittenberg University, Springfield, OH, USA. ${ }^{380}$ School of Applied Psychology, ZHAW Zurich University of Applied Sciences, Winterthur, Switzerland. ${ }^{381}$ Athens University of Economic and Business, Athens, Greece. ${ }^{382}$ Black Dog Institute, UNSW Sydney, Sydney, Australia. ${ }^{383}$ Emotional Health for All Foundation, Jakarta, Indonesia. ${ }^{384}$ University of Virginia, Denver, CO, USA. ${ }^{385}$ Department of Psychology, Ashland University, Ashland, OH, USA. ${ }^{386}$ Department of Psychology, Ashland University, Medina, OH, USA. ${ }^{387}$ Department of Psychology, Tufts University, Medford, MA, USA. ${ }^{888}$ Harrisburg University of Science and Technology, Bethlehem, PA, USA. ${ }^{889}$ Stanford University, Stanford, CA, USA. ${ }^{39}$ United States International University-Africa, Nairobi, Kenya. ${ }^{391}$ Université Grenoble Alpes; Institut Universitaire de France, Grenoble, France. ${ }^{392}$ Department of Psychology, University of Wisconsin-Madison, Madison, WI, USA. $\varpi_{\text {e-mail: millerj@willamette.edu }}$ 


\section{Reporting Summary}

Nature Portfolio wishes to improve the reproducibility of the work that we publish. This form provides structure for consistency and transparency in reporting. For further information on Nature Portfolio policies, see our Editorial Policies and the Editorial Policy Checklist.

\section{Statistics}

For all statistical analyses, confirm that the following items are present in the figure legend, table legend, main text, or Methods section.

n/a Confirmed

$\bigotimes$ The exact sample size $(n)$ for each experimental group/condition, given as a discrete number and unit of measurement

$\bigotimes$ A statement on whether measurements were taken from distinct samples or whether the same sample was measured repeatedly

$\varnothing$ The statistical test(s) used AND whether they are one- or two-sided

Only common tests should be described solely by name; describe more complex techniques in the Methods section.

$\bigotimes$ A description of all covariates tested

$\bigotimes$ A description of any assumptions or corrections, such as tests of normality and adjustment for multiple comparisons

$\checkmark$ A full description of the statistical parameters including central tendency (e.g. means) or other basic estimates (e.g. regression coefficient)

$\triangle$ AND variation (e.g. standard deviation) or associated estimates of uncertainty (e.g. confidence intervals)

For null hypothesis testing, the test statistic (e.g. $F, t, r$ ) with confidence intervals, effect sizes, degrees of freedom and $P$ value noted

$\triangle$ Give $P$ values as exact values whenever suitable.

$\bigotimes$ For Bayesian analysis, information on the choice of priors and Markov chain Monte Carlo settings

$\bigotimes$ For hierarchical and complex designs, identification of the appropriate level for tests and full reporting of outcomes

$\bigotimes$ Estimates of effect sizes (e.g. Cohen's $d$, Pearson's $r$ ), indicating how they were calculated

Our web collection on statistics for biologists contains articles on many of the points above.

\section{Software and code}

Policy information about availability of computer code

Data collection The data collection was implemented using the formr software framework. Details regarding implementation can be found here: https:// osf.io/shn5r/.

Data analysis All data analysis was completed using R. Scripts are available at https://osf.io/4yf9d/.

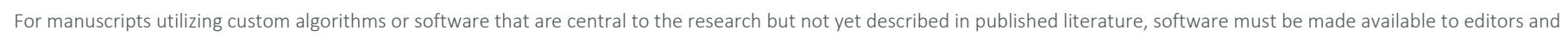

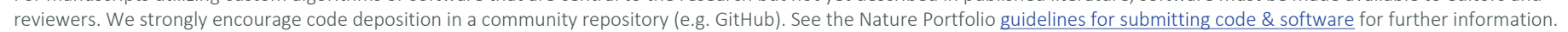

\section{Data}

Policy information about availability of data

All manuscripts must include a data availability statement. This statement should provide the following information, where applicable:

- Accession codes, unique identifiers, or web links for publicly available datasets

- A description of any restrictions on data availability

- For clinical datasets or third party data, please ensure that the statement adheres to our policy 


\section{Field-specific reporting}

Please select the one below that is the best fit for your research. If you are not sure, read the appropriate sections before making your selection.

Life sciences

Хehavioural \& social sciences

Ecological, evolutionary \& environmental sciences

For a reference copy of the document with all sections, see nature.com/documents/nr-reporting-summary-flat.pdf

\section{Behavioural \& social sciences study design}

All studies must disclose on these points even when the disclosure is negative.

Study description

Research sample

Sampling strategy

Data collection

Timing

Data exclusions

Non-participation

Randomization quantitative experimental

We collected 27,989 responses from May 2020 to October 2020. After implementing preregistered exclusions (see details at https:// doi.org/10.6084/m9.figshare.c.4878591.v1) and an additional exclusion of nine duplicate IDs, our final sample included 21,644 participants from 87 countries/regions $(63.41 \%$ female, $35.34 \%$ male, $0.45 \%$ other genders, $0.56 \%$ preferred not to say, and $0.24 \%$ missing responses to the gender question; $\mathrm{M}$ age $=31.91$, SD age $=14.52$; see Supplementary Table 1 for sample size per country/ region and Supplementary Table 2 for sample size per month). Of the 87 countries/regions represented, 37 had over 200 participants, surpassing our $95 \%$ power criterion based on simulations in our power analysis.

We used a combination of convenience sampling and semi-representative panelling.

We conducted a simulation study to estimate power for a variety of potential effect sizes $(|d|=0.05$ to 0.29 , separated by increments of 0.02), number of countries/regions (Ncountry/region $=30,35,40,45,50,55,60$ ), within-country/region sample sizes $(N=200,400,600,800)$, by-country/region intercept variances ( $\sigma 2$ intercept $=0.05,0.30,0.55,0.80$ ), and by-country/region slope variances ( $\sigma 2$ slope $=0.0,0.02,0.03,0.04)$ at $\alpha=.017$. The lowest level of intercept variances in our simulation was chosen on the basis of an ongoing multi-country/region project tracking rates of depression ( $\sigma 2$ intercept $=0.04$ ) and worries about the COVID-19 ( $\sigma 2$ intercept $=0.06$ ) across countries/regions during the COVID-19 outbreak (See Supplementary Information for details). The lowest level of slope variances in our simulation was chosen on the basis of the average slope variance ( $\sigma 2$ slope $<0.01)$ in a large multi-site, multi-country/region project involving 28 psychological manipulations 125 . The slope variances capture the variability of the effect of psychological manipulations, and there is no apparent reason to expect that the effect of reappraisal interventions on emotions is more variable than most other psychological manipulations in Klein et al.. In fact, appraisal theories of emotion argue that the relationship between appraisals and emotions is culturally universal, suggesting low variability. As one example to show that similar appraisals associate with similar emotional experiences, we found the associations varied little across countries/regions between perceived insufficient government response and depression ( $\sigma 2$ slope $=0.003$ ) and between perceived insufficient government response and worries ( 02 slope $=0.003$ ) during the COVID-19 pandemic (See Supplementary Information for details), consistent with the observation of low slope variances $(\sigma 2 s$ lope $<0.01$ ) in Klein et al. Despite expecting low variability from empirical findings and theories, we tested a variety of intercept variances and slope variances in our power simulation, some of which were much higher than those in the Klein et al. and Fetzer et al. to be maximally conservative. We conducted 1000 simulations for each set of simulation parameters using the simr package using computing power harnessed through the Open Science Grid.

Data was collected via an online survey, using the formr software framework. Participants clicked a single data collection link that led to either the current study or the other two studies in the COVID-19 Rapid Project. Participants completed the experiments at their own device and no experimenter was present during data collection.

Data collection began May 6, 2020 and finished October 23, 2020.

We excluded 6345 responses after implementing preregistered exclusions (see details at https://doi.org/10.6084/ m9.figshare.c.4878591.v1) and an additional exclusion of nine duplicate IDs.

We did not formally examine dropouts, but we preregistered to exclude participants who completed fewer than $50 \%$ of the questions in the study. We found that the passive control condition had fewer such participants (16.17\%) than the other three conditions (23.86\% in the active control condition, $24.41 \%$ in the reconstrual condition, and $23.90 \%$ in the repurposing condition), Holm's adjusted Ps $<0.001$. One possible explanation for this difference is that the instructions given to participants in the passive control condition were shorter than those given in the other conditions, requiring less cognitive effort to read and less time to complete the study.

\section{Reporting for specific materials, systems and methods}

We require information from authors about some types of materials, experimental systems and methods used in many studies. Here, indicate whether each material, system or method listed is relevant to your study. If you are not sure if a list item applies to your research, read the appropriate section before selecting a response. 


\begin{tabular}{l|l}
\hline n/a & Involved in the study \\
$\square$ & $\square$ Antibodies \\
$\square$ & $\square$ Eukaryotic cell lines \\
$\square$ & $\square$ Palaeontology and archaeology \\
$\square$ & $\square$ Animals and other organisms \\
$\square$ & $\square$ Clinical data \\
$\square$ & $\square$ Dual use research of concern
\end{tabular}
n/a Involved in the study

\section{Human research participants}

\section{Policy information about studies involving human research participants}

\section{Population characteristics}

Recruitment

\section{Ethics oversight}

\section{See above}

Participants were recruited by the PSA network. The PSA recruited 186 member labs from 55 countries/regions speaking 42 languages. Of the 27,989 participants recruited to complete the current study (not counting participants for the other two studies in the PSA COVID-19 Rapid Project), 4,050 of them were recruited through semi-representative paneling (based on sex, age, and sometimes ethnicity) from the following countries/regions: Egypt, Kenya, Nigeria, South Africa, Mexico, United States, Austria, Romania, Russia, Sweden, Switzerland, United Kingdom, China, Japan, and South Korea (270 participants per country/region). The remaining participants were recruited through the research groups by convenience sampling. Although the specifics of the consent procedure differed across research groups, all participants provided informed consent. The style and the amount of compensation varied with local conventions. More information regarding participant compensation and sample size can be found at https://psyarxiv.com/x976j/. Our sample was not nationally representative within each country/ region, and it appeared to over-represent females, younger people, and people with internet access.

Primary ethics approval was provided by the Institutional Review Board at Ashland University, additional approvals were recorded as necessary depending on regional and national policies across our worldwide sample. Detailed information regarding IRB approval is available here: https://osf.io/dq846/. Each research group obtained approval from their local Ethics Committee or IRB to conduct the study, explicitly indicated that their institution did not require approval for the researchers

to conduct this type of task, or explicitly indicated that the current study was covered by a pre-existing approval. 